\title{
The emission lines of quasars and similar objects
}

\author{
Kris Davidson* \\ Astronomy Department, University of Minnesota, Minneapolis, MN 55455 \\ Hagai Netzer ${ }^{\dagger}$ \\ Department of Astronomy, University of Texas, Austin, $T X 78712$
}

Much of our information about quasars is derived from their emission-line spectra. The analysis of such spectra has become an intricate subject which differs considerably from traditional, low-density nebular astrophysics. This review is intended to explain our present understanding of the situation, including some aspects of galactic nuclei whose luminosities are more modest than quasars. Quasars' line-emitting regions are probably photoionized (even if supplementary heating processes also occur). So far, models have been constructed which include ionization and thermal equilibria, the transfer of resonance-line and related photons, and the likely effects of absorption and scattering by dust grains. From comparisons between emission-line intensities produced in these models and observed quasars' spectra, it appears that certain densities and pressures and size scales occur in or around quasars. The relative abundances of elements are not very far from solar values, although it is suspected that heavy elements-carbon, nitrogen, and oxygen in particular-are moderately "overabundant" in quasars. The emission-line intensities also provide indirect information about quasars' ultraviolet and soft-x-ray continua; there are hints that photons with energies between 20 and $300 \mathrm{eV}$-which are not directly observable-may even represent the peak of the luminous output of a typical quasar. Finally, some gas-dynamical questions, while extremely important, are very difficult to answer, because of a lack of observables.

\section{CONTENTS}

I. Introduction
A. Some historical remarks
B. Quasars, as observed optically
C. Topics not included in this review
D. Topics included in this review

II. Photoionized Nebulae-A Brief Overview

A. The photoionization equilibrium: Counting
the photons

B. Thermal equilibrium: Counting the ergs 724

C. The emergent spectrum

D. Where to find ionic data

III. Conventional, Dustless Photoionization Models for Quasars' Spectra
A. Historical and preliminary
B. A specific photoionization calculation
C. Varying the assumed parameters

IV. Line Transfer
A. General considerations
B. Possible suppression of resonance emission lines
C. The hydrogen line spectrum
D. The helium line spectrum
E. Fluorescent processes

V. Dust and Quasars

$\begin{array}{ll}\text { A. Correlation of visual and ultraviolet data } & 743 \\ \text { B. Dust in our Galaxy } & 745 \\ \text { C. Intergalactic dust? } & 745 \\ \text { D. Dust in quasars' galaxies } & 746 \\ \text { E. Dust in quasars } & 746 \\ \text { F. What is the intrinsic spectrum of a quasar? } & 747 \\ \text { G. Comparing lines with continua } & 748 \\ \text { Some Additional Spectral Features } & 750 \\ \text { A. Forbidden lines } & 750 \\ \text { B. FeII lines } & 751 \\ \text { C. The " } 3000 \AA \text { A bump" } & 752\end{array}$

\footnotetext{
*Alfred P. Sloan Foundation Research Fellow, 1975-1979.

**On leave from the Dept. of Physics and Astronomy, Tel Aviv University.
}

VII. Some Additional Possibilities

A. Concerning the absence of broad forbidden lines

B. Can densities be surprisingly high?

C. Additional heating processes

D. Nonthermal emission lines?

E. Emission-line variability

VIII. Gas Motions and Dynamics; Line Profiles

A. Generalities

B. Acceleration of gas - mainly by radiation pressure

C. Emission line profiles

D. More remarks about certain parameters Acknowledgments

References

\section{INTRODUCTION}

\section{A. Some historical remarks}

Like other modern astronomical subjects, the study of quasars has been greatly affected by fashion and by chance; Weedman (1976a) has reasonably argued that these influences have sometimes led to misplaced attention and unnecessary debates.

The first quasars to be noticed were some radio sources that were identified, during the early 1960 s, with optically observable "stars" (see Schmidt, 1963; Oke, 1963; Greenstein and Mathews, 1963; and Burbidge and Burbidge, 1967). When it was recognized that these were really not stars in our Galaxy, they became known as "quasistellar objects" or "QSO's" - or today, quasars. Their near-ultraviolet, visual, and near-infrared spectra were found to be continua plus a few emission lines, red-shifted like the spectra of very distant galaxies; if the quasars' red shifts, like those of galaxies, indicated cosmological distances, then their intrinsic luminosities must considerably exceed

Copyright $\odot 1979$ American Physical Society 
those of galaxies. However, some quasars were seen to fluctuate in brightness within a few years or less; this meant, according to arguments involving lighttravel times, that the luminous souces must be smaller than a few light-years across, tiny compared with any galaxy and far too small to be resolved optically. This inspired a lengthy dispute about whether quasars' red shifts are really cosmological (see Field, Arp, and Bahcall, 1973).

But long before any quasars had been noticed, a few galaxies, called "Seyfert galaxies," were known to have luminous, compact nuclei, with emission lines in their spectra. Often mentioned in connection with quasars during the $1960 \mathrm{~s}$, Seyfert galaxies assumed new importance when some of their central brightnesses were seen to fluctuate like quasars (see references cited by Weedman, 1976a, 1977). Today it seems probable that quasars are like a certain class of Seyfert nuclei, but more luminous; they are probably at the centers of galaxies (which are detectable in some cases-see Morton, Williams, and Green, 1978; Wehinger and Wyckoff, 1978; Hawkins, 1978; and earlier references cited by these authors, as well as comments by Bahcall, 1971), and their emission-line red shifts do indeed indicate cosmological distances.

Although it was their radio emission that drew attention to the first-known quasars, today we know that most quasars are not prominent radio sources (Sandage and Luyten, 1969; Lynds and Wills, 1972; Osmer and Smith, 1977a; Murdoch and Crawford, 1977; Sramek and Weedman, 1978). Of the nearly 1000 presently known quasars, several hundred were first noticed as radio objects, but only a minority of these are strong radio sources. Among those which were first noticed at optical wavelengths, only a small fraction-10\% to $30 \%$ - can be detected with present-day radio telescopes. Even in a "radio-bright" quasar, the visual-wavelength radiation usually carries significantly more energy than the radio emission. But this is not to say that these objects are predominantly visual-wavelength emitters; their infrared, ultraviolet, $x-r a y$, and perhaps even $\gamma$-ray fluxes are also important.

At visual wavelengths the most conspicuous quasar is a "12th magnitude" object, i.e., it seems about $1 / 300$ th as bright as a faint naked-eye star; most of the other known quasars are of the 15th to 20th magnitude, 10 to 1000 times fainter. Being so faint, quasars that fail to draw attention as radio emitters are difficult to identify, because on most photographs they look just like the equally faint foreground stars, which are multitudinous. Sometimes colors have been useful (most quasars are "bluer," i.e., tend to emit at shorter wavelengths, than most stars), but generally the emission lines are necessary for identification; it has become possible to use special photographs on which each image is actually a small spectrum, so that quasars can be picked out because of their emission lines (see references cited by Smith, 1978). Even with this technique only a small fraction of the sky has been examined, and it appears likely that the total number of quasars down to 20th magnitude is of the order of $10^{5}$ or $10^{6}$.

Most known Seyfert galaxies have been found more easily, because they are close enough to be recognizable as galaxies. But only a small fraction of galaxies are Seyferts, and as with quasars, spectra are required for identification.

The distances of the nearest quasars, estimated from their red shifts, are of the order of $10^{9}$ light-years; while the distances of the farthest ones are comparable to the size of the observable universe. Considering light-travel times, this means that we see the more distant objects as they were moderately early in the history of the universe, not long after the first galaxies formed. Although we do not know much about quasars' lifetimes, it is probable that the early ones are now extinct. Quasars span such a wide range of intrinsic luminosities that some of the more distant ones appear as bright as some of the nearest. The nuclei of Seyfert galaxies are intrinsically less luminous than most quasars but more luminous than most ordinary galaxies.

Many theorists have "explained" the radio, infrared, visual, ultraviolet, x-ray, and $\gamma$-ray continua of quasars by invoking nonthermal processes such as synchrotron radiation (magnetobremsstrahlung) and the inverse Compton effect, while the basic energy sources are suspected to be gravitational fields of black holes (see Sec. I.C below). But the details of these explanations are unsatisfying, because many parameters are both important and unobservable. There must be supplies of energetic electrons, accelerated by mechanisms that are imperfectly understood (!), in magnetic fields whose geometries are model dependent; one must guess whether the electron-energy spectra are correlated with magnetic field strengths, and how the electrons' velocities might be correlated in direction with magnetic field lines; the relativistic electrons may lose most of their energies to radiation, or they may escape; energetic protons and other massive particles or ions may or may not contain as much total energy as the electrons; magnetic fields may be quasistatic, or there may be strong low-frequency electromagnetic waves; and so on. Even though the theoretical situation is so complex, it produces few observable quantities: usually only a total flux, a few tentative statements about the shape of the emitted continuum, and perhaps statements about linear or circular polarization; and these results may not follow uniquely from any one particular model.

Thus, being dissatisfied with a largely theoretical nonthermal continuum approach, we think that emission lines are presently more useful for learning about quasars. The emission-line spectra have several good attributes. First, there are a number of individual lines whose relative intensities can be measured, giving a fair number of well-defined observables. The line profiles, although more difficult to measure, may also be useful. Second, the physical mechanisms involved in producing the lines are fairly well understood, and geometrical assumptions, while significant, are not quite as crucial as in models for nonthermal continua. Third, the emission-line spectra of most quasars are surprisingly alike, with a few interesting exceptions. There are other motives for investigating the emission lines. In Sec. $\mathrm{V}$ we shall express a suspicion that the true continua cannot be known until the lines are under- 
stood. The line-emitting regions may be related to the mysterious supplies of "fuel" (gravitationally accreted material) which are required in most theoretical pictures of quasars. And it is from the emission lines that we learn of the occurrence of postprimordial nucleosynthesis even in the earliest, highest-red-shift objects yet seen: they are (or were) rich in elements heavier than helium. For all of these reasons, we think that the emission lines deserve a high priority in the study of quasars.

Unfortunately, analyses of these emission-line spectra are complicated, have been considered in realistic detail by only a few people, and have not been given adequate attention in any published review; hence the following review. This account will be concerned largely with models of line-emitting regions, and how such models can be used to extract information from the observed spectra; many observational details will be omitted. Even so, the topic is lengthy.

\section{B. Quasars, as observed optically}

We shall not be very careful here about what we call a quasar. Without arguing the point, we suppose that the nuclei of "Type 1" Seyfert galaxies - those with broad emission lines-are modest quasars; but if this assumption is wrong, only a few details of our discussion will be invalid as a consequence. The Seyferts are spiral galaxies with prominent, compact nuclei; for descriptions of them and their resemblances to quasars, see Weedman (1977) and references therein. There are other luminous objects, called "N-type galaxies" and "broad-line radio galaxies," which share many of the characteristics of Seyferts and quasars (in fact, some quasars are probably in N-type galaxies); for details, see references cited by Osterbrock and Grandi (1979). “BL Lacertae objects" are almost (if not precisely) quasars without prominent emission lines; see Stein, O'Dell, and Strittmatter (1976), Miller (1979), and the Proceedings of the 1978 Pittsburgh conference on BL Lac objects (edited by A. Wolfe). Rowan-Robinson $(1976,1977)$ has discussed some speculative generalizations about these various classes, which together are often called "active galactic nuclei."

For interesting reviews of observational data on quasars, see Smith (1978) and Weedman (1976); older, more traditional reviews are those by Burbidge and Burbidge (1967) and Schmidt (1969). Regarding observations of emission lines in particular, see Baldwin (1978), Neugebauer et al. (1979), and Strittmatter and Williams (1976). For a list of several hundred quasars, with some data and many references, see Burbidge, Crowne, and Smith (1977). Quasars have been catalogued in many ways, so that different objects often have dissimilar-

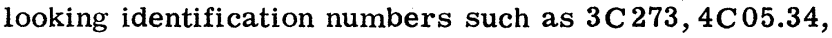
PHL 938, PKS 0237-23, etc.; these individual tags will not matter here.

The foremost observational parameter of a quasar is its emission-line red shift $z$ : a line with laboratory ("rest") wavelength $\lambda_{0}$ is observed at wavelength $\lambda_{\text {apparent }}=(1+z) \lambda_{0}$, where $z>0$ because of the expansion of the universe. The object's distance is proportional to $z$ for $z \ll 1$ and in general depends upon cosmological parameters. The nearest quasars have $z$ somewhat less than 0.1 (which corresponds to a distance of roughly $1.5 \times 10^{9}$ light-years), while $z$ exceeds 3 for the most distant examples. Because of the Earth's atmosphere and instrumental detection efficiencies, ground-based observations of emission lines have mostly been restricted to the apparent wavelength range 3000-9000 corresponding, for example, to rest wavelengths $1000-3000 \AA$ in a case where $z=2$. Thus, until recently, quasars' spectra at visual rest wavelengths were known only for low-red-shift objects while the intrinsic ultraviolet spectra were known only for high-red-shift objects. This was potentially dangerous, because the distant, high-red-shift quasars, occurring relatively early in the history of the universe, might have been quite different from nearby, low-red-shift, present-day objects. Fortunately, ultraviolet spectra, obtained with satellite- or rocket-borne instruments, and also some infrared data have begun to appear since 1976, and so far there is no evidence that high - and low-red-shift quasars' spectra are very different.

Estimating a quasar's distance from its red shift, and combining this with its apparent brightness in some observable wavelength range, we can find the intrinsic luminosity (radiated power) in that range. As an orderof-magnitude approximation, at least some quasars' luminosities are evenly distributed among the nearinfrared $(1-5 \mu \mathrm{m})$, visual (3000-9000 $⿱$ ) , ultraviolet (500-2500 $\AA$ ), and $x$-ray $(1-10 \mathrm{keV})$ ranges. (We do not know whether something profound or simple is behind this remarkable fact.) Total luminosities are often between $10^{46}$ and $10^{48} \mathrm{erg} \mathrm{s}^{-1}$, although Seyfert galaxies may be less luminous and a few very luminous highred-shift quasars at least temporarily exceed $10^{48}$ $\mathrm{erg} \mathrm{s}^{-1}$. For comparison, the luminosity of the Sun is $10^{33.6} \mathrm{erg} \mathrm{s}^{-1}$ and a fairly respectable galaxy may produce $10^{43}$ or $10^{44} \mathrm{erg} \mathrm{s}^{-1}$.

As mentioned above, although they are far more luminous than galaxies, quasars are much smaller than galaxies and may exist within galaxies. Some (though not all) quasars' continua have varied within a few years (e.g., see Neugebauer et al., 1979; Scott et al., 1976; Heckmann, 1976; McGimsey et al., 1975; Liller and Liller, 1975; Grandi and Tifft, 1974; Angione, 1973; and other references to be cited later in this review). In an extreme case, a quasar can vary in brightness by a factor of more than 10 in less (sometimes much less) than a year. However, these fluctuations generally involve only the continuum; the emission lines remain fairly steady except in some Seyfert nuclei with relatively modest luminosities. We also note that most quasars (especially the "radio-quiet" ones) may be fairly steady over time scales up to at least 50 years. It is conceivable that the very far ultraviolet and soft$x$-ray continua may be steady even where the visual, moderate ultraviolet, and moderately hard-x-ray fluxes vary (this may be relevant to some of our discussion in Sec. V).

Let $F(\varepsilon) d \varepsilon$ denote an energy flux in photon-energy in- 
terval $d \varepsilon .^{1}$ It has been customary to represent a typical quasar's infrared-ultraviolet continuum as a power law, $F(\varepsilon) \sim \varepsilon^{-s}$, with $s$ close to unity. However, Fig. 1, which is taken from Neugebauer, Oke, Becklin, and Mathews (1979), shows that this is usually an oversimplification. Quasars' continua are often "bumpy" and curved on a $\log F(\varepsilon)-\log \varepsilon$ plot. These continua are probably nonthermal in origin, i.e., due to processes like synchrotron radiation and inverse Compton scattering; but there may be thermal exceptions in the infrared, far ultraviolet, and soft-x-ray regions of the spectrum.

Modern observations of quasars' emission lines have been described by Baldwin $(1975,1978)$, Osmer and Smith $(1976,1977 \mathrm{a})$, Baldwin and Netzer (1978), and others; similar observations of Seyfert galaxies and related objects have been described by Adams and Weedman (1975), Osterbrock (1977), Grandi and Osterbrock (1978), and Phillips (1978a). Figure 2 shows, for example, a spectrum of a low-red-shift quasar with $z=0.206$. The emission lines in this spectrum are familiar to astronomers, because many gaseous nebulae in our Galaxy show the same lines. However, unlike an ordinary nebular spectrum, some of the quasar's lines are quite broad. These are the "permitted" lines, including the Balmer series of hydrogen. The linewidths are probably due to differential Doppler shifts, involving large-scale velocity dispersions in the line-emitting material. Some other lines - "forbidden lines"-are relatively narrow (narrower, in fact, than Fig. 2 shows, since instrumental broadening is present); we shall explain the likely reasons for this difference later. Figure 3 shows the spectrum of a Seyfert galaxy, which in this particular case differs from the quasar's spectrum in two qualitative respects: the Balmer lines have asymmetric shapes, and there are strong features involving many lines of singly ionized iron. Figure 4 shows the ultraviolet spectra of several high-red-shift quasars. Most of the ultraviolet emission lines can be identified with little difficulty; the most prominent lines involve hydrogen, $\mathrm{C}^{2+}, \mathrm{C}^{3+}$, and $\mathrm{Mg}^{+}$ions. There are no narrow ultraviolet forbidden lines, and the different quasars' spectra in Fig. 4 resemble each other.

Much of this review will be a discussion of how we can derive physical information--gas and energy densities, chemical compositions, sizes of emitting regions-from the relative intensities of spectral lines shown in Figs. 2, 3, and 4. Although the results will be imprecise, we think that there is more derivable information in the emission lines than in the perhaps featureless, perhaps "bumpy" continua.

\section{Topics not included in this review}

We shall not discuss in detail the many theories that have been invented to explain the origins, energy supp-

\footnotetext{
${ }^{1}$ We shall usually discuss photon energies $\varepsilon$ and wavelengths $\lambda$, although some astronomers refer to frequencies $\nu$ even in the optical range. For convenience, note that a photon energy $\varepsilon=10 \mathrm{eV}$, near the important Lyman-alpha emission line, corresponds to $\lambda=1240 \AA$ and $\nu=10^{15.38} \mathrm{~Hz}$. In this review, $\varepsilon$ and $\lambda$ will usually be "rest" values, i.e., as measured in the reference frame of the quasar.
}

lies, and continuum luminosities of quasars. Many rough theoretical ideas about quasars are plausible, but the more detailed scenarios are quite speculative and do not appear particularly susceptible to direct observational tests. Several descriptions of the topic, from what is lately a conventional point of view, have been given by Rees $(1977 a, b ; 1978 a, b)$. Many galaxies have central nuclei-massive, dense star clusters, very much more compact than the galaxies-and most theories of quasars have involved such galactic nuclei. If a galactic nucleus is dense enough, considerable amounts of gas must be released in supernova explosions, stellar collisions, and other catastrophic events in the nucleus. If this gas does not escape, it may form new stars, or, more likely, will tend to collect at the center of the galactic nucleus, presumably in some sort of disklike configuration (because its angular momentum is significant). Perhaps a central, massive black hole will form, surrounded by a rotating disk of gas which spirals into the black hole because of viscous effects (probably involving turbulence). This leads to a model proposed by Lynden-Bell (1969) which has become rather standard (although its truth has not been proven). [See also Lynden-Bell (1978).] The total gravitational energy converted into luminosity is probably of the order of $0.1 c^{2}$ per unit mass which falls into the black hole; this means that an accretion rate of $10^{27} \mathrm{~g} \mathrm{~s}^{-1}$, about 10 solar masses per year, can provide a quasarlike luminosity of the order of $10^{47} \mathrm{erg} \mathrm{s}^{-1}$. However, the details of the conversion to a continuum spectrum are not at all obvious. Events in the surrounding region-the dense star cluster-must supply the accreted mass; stellar collisions and near-collisions, supernova explosions, and tidal disruptions of stars passing close to the black hole may be important. One can argue that the most important problems regarding quasars, their origins and sustenance, involve the surrounding regions rather than the hypothetical central black holes. The line-emitting gas, to be discussed in this review, occurs in or near these regions.

It appears that a quasar can best be represented by a "kitchen sink" model, with a drainpipe (the black hole) and many interesting items piled up above the drainpipe (stars, pulsars, small black holes, gas clouds, electromagnetic fields); but there are many ways to choose and arrange the piled-up items. Plasma-physics effects (e.g., see Lovelace, 1976) and explosive events (e.g., see Blandford and McKee, 1977) may occur in the accretion vortex. For a variety of complicated ideas about this complicated subject, see LyndenBell and Rees (1971), Arons, Kulsrud, and Ostriker (1975), von Hoerner and Saslaw (1976), Ginzburg and Ozernoi (1977), Young, Shields, and Wheeler (1977), Bailey and Clube (1978), Hills (1978), Keenan (1978), Rowan-Robinson (1977), and Begelman and Rees (1978).

Another topic that we shall avoid is the study of quasars at radio wavelengths. In some cases, activity in quasars can be resolved by radio techniques (whose angular resolution is now better, in some cases, than that of optical astronomy), and some information follows from the radio continua. However, the energy fluxes involved are only small fractions of quasars' 

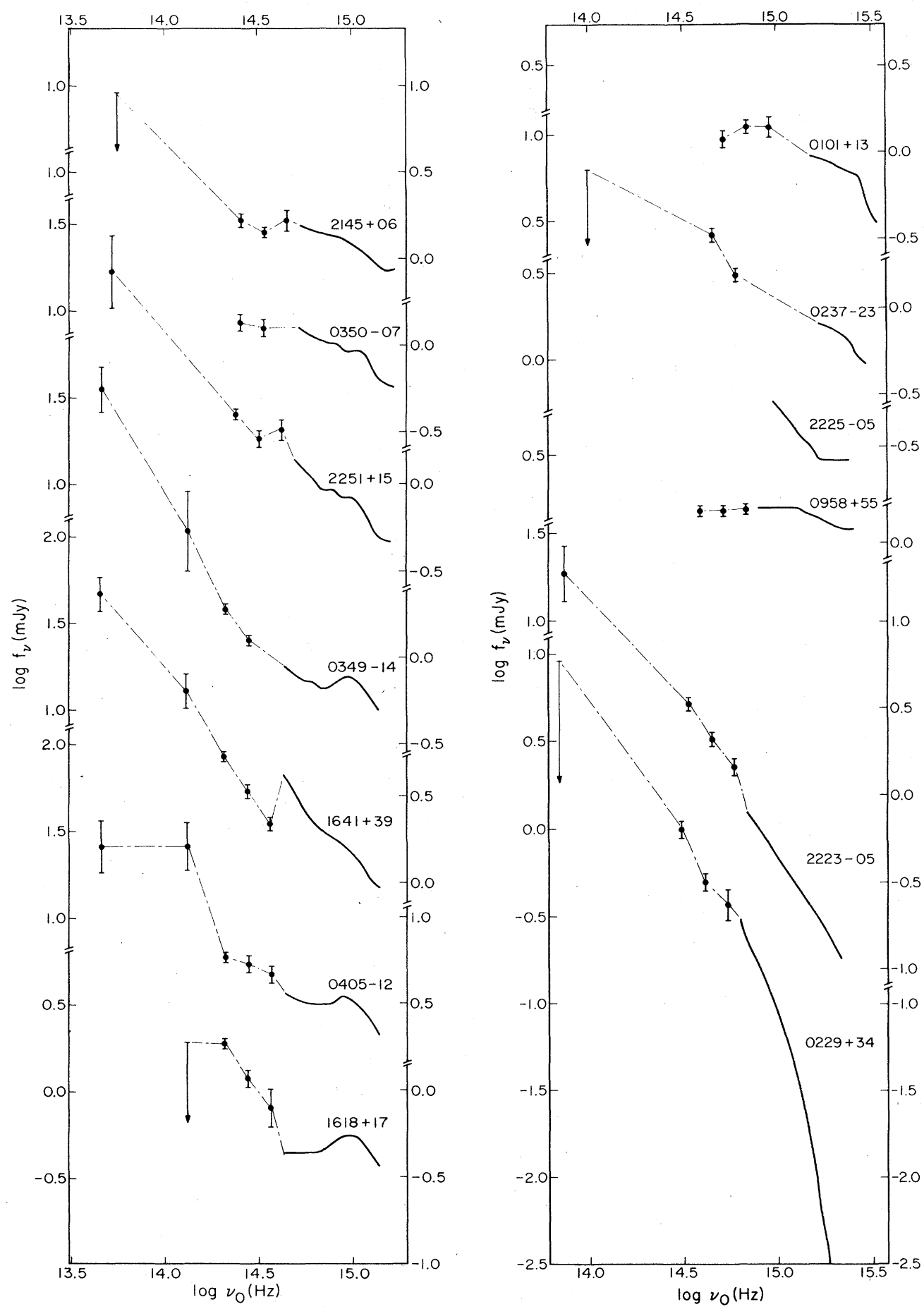

FIG. 1. $\log f_{\nu}-\log \nu$ plots of the infrared-to-ultraviolet continuum spectra of several quasars, according to Neugebauer, Oke, Becklin, and Mathews (1979). Emission lines are mostly not included. Frequencies $\nu_{0}$ are in the quasars' "rest" (intrinsic) frames. (Note that $\nu=10^{15} \mathrm{~Hz}$ corresponds to $\varepsilon=4.1 \mathrm{eV}$ or $\lambda=3000 \AA=0.3 \mu \mathrm{m}$.) Copyright The Astrophysical Journal.

total outputs, the precise implications of the radio observations are model dependent, and most quasars are difficult to detect at radio wavelengths. See Kellerman (1974, 1978), Burbidge, Jones, and O'Dell (1974), Jones, O'Dell, and Stein (1974), and Miley and Miller (1979).

We shall not discuss $\mathrm{x}$-ray and $\gamma$-ray $(\varepsilon \sim 1 \mathrm{keV}$ to
$1 \mathrm{GeV}$ ) observations and theory. Some quasars have appreciable fractions of their luminosities in this range; see Culhane (1978), Elvis et al. (1978), Giacconi (1978), Marshall et al. (1978), Stark, Burnell, and Culhane (1978), Swanenberg et al. (1978), Barr et al. (1977), DiCocco et al. (1977), and Gursky and Schwartz (1977). In general, we shall not discuss the origins 


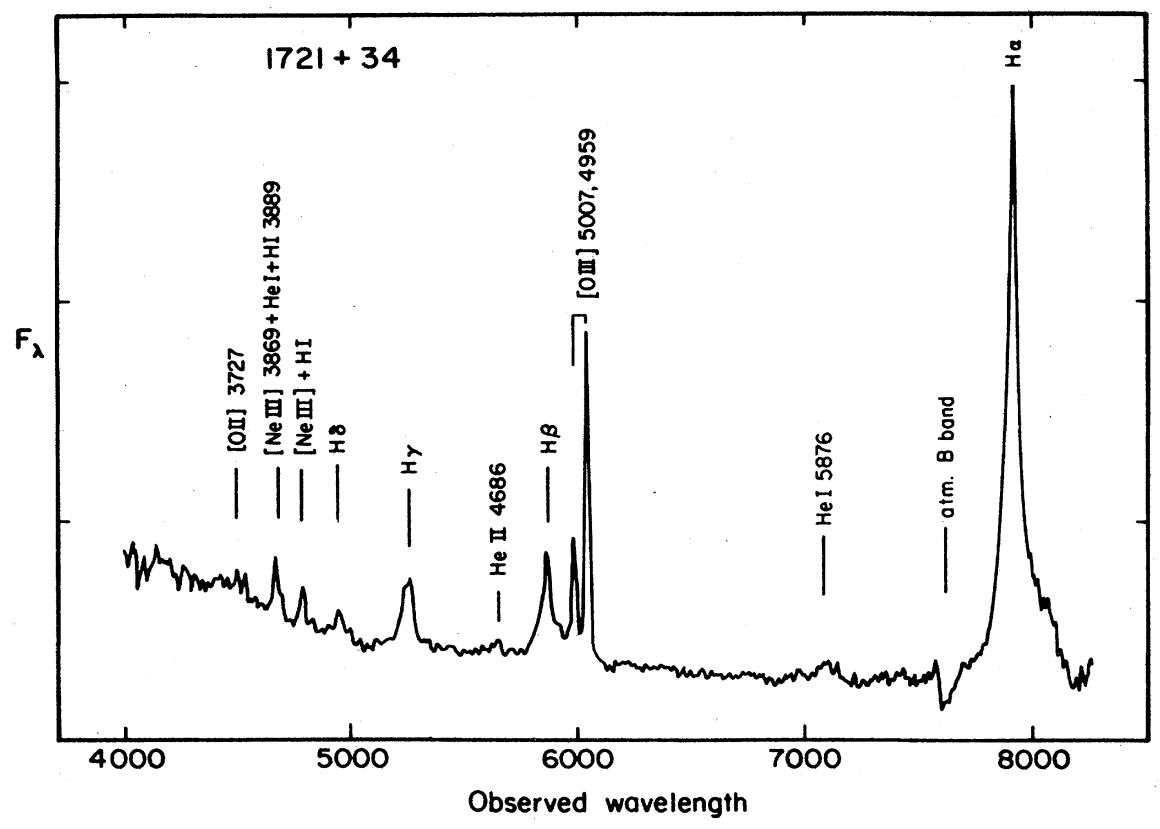

FIG. 2. The visual-wavelength spectrum of a typical low-redshift quasar with $z=0.2$, obtained by D. Wills and B. Wills at MacDonald Observatory. (Note that the abscissas here are apparent wavelengths, rather than "rest" wavelengths. The absorption feature near $7600 \AA$ is due to the terrestrial atmosphere.)

of nonthermal continua in any wavelength range.

A typical high-red-shift quasar's spectrum has many narrow absorption lines, especially including the Lyman-alpha line of atomic hydrogen, with many different red shifts $z_{a b}$. Almost all of the absorption red shifts are less than the quasar's emission red shift $z_{e m}$; some are close to $z_{e m}$ but others are not. For example, in a spectrum with $z_{e m}=2.00$, one "absorption system" might have $z_{a b}=1.95$ (which may correspond to a difference of several thousand $\mathrm{km} \mathrm{s}^{-1}$ between the line-of-
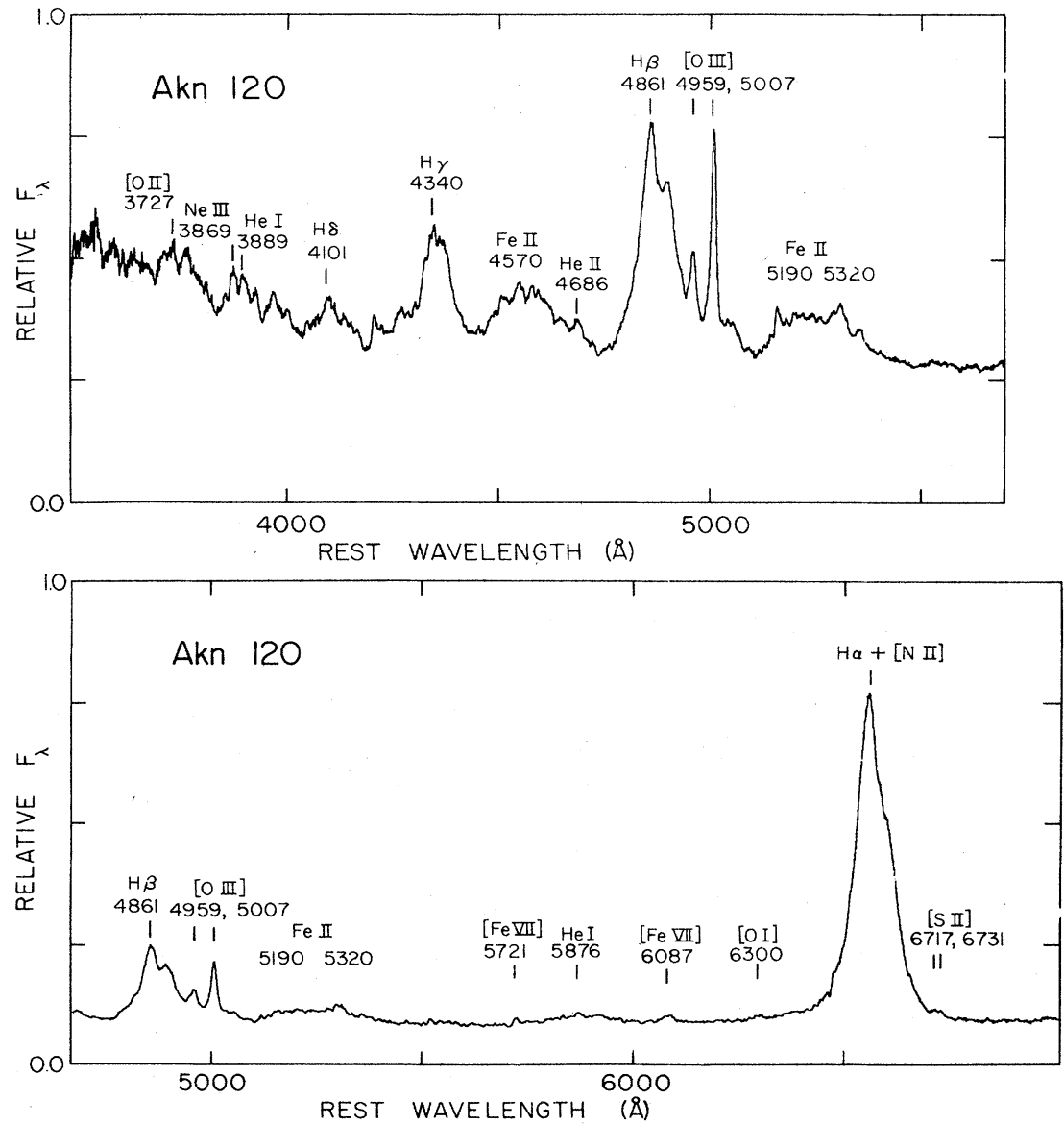

FIG. 3. The visual-wavelength spectrum of a "Type 1" Seyfert galaxy with strong Fe II emission, from Osterbrock and Phillips (1977). Courtesy of the Publications of the Astronomical Society of the Pacific. 


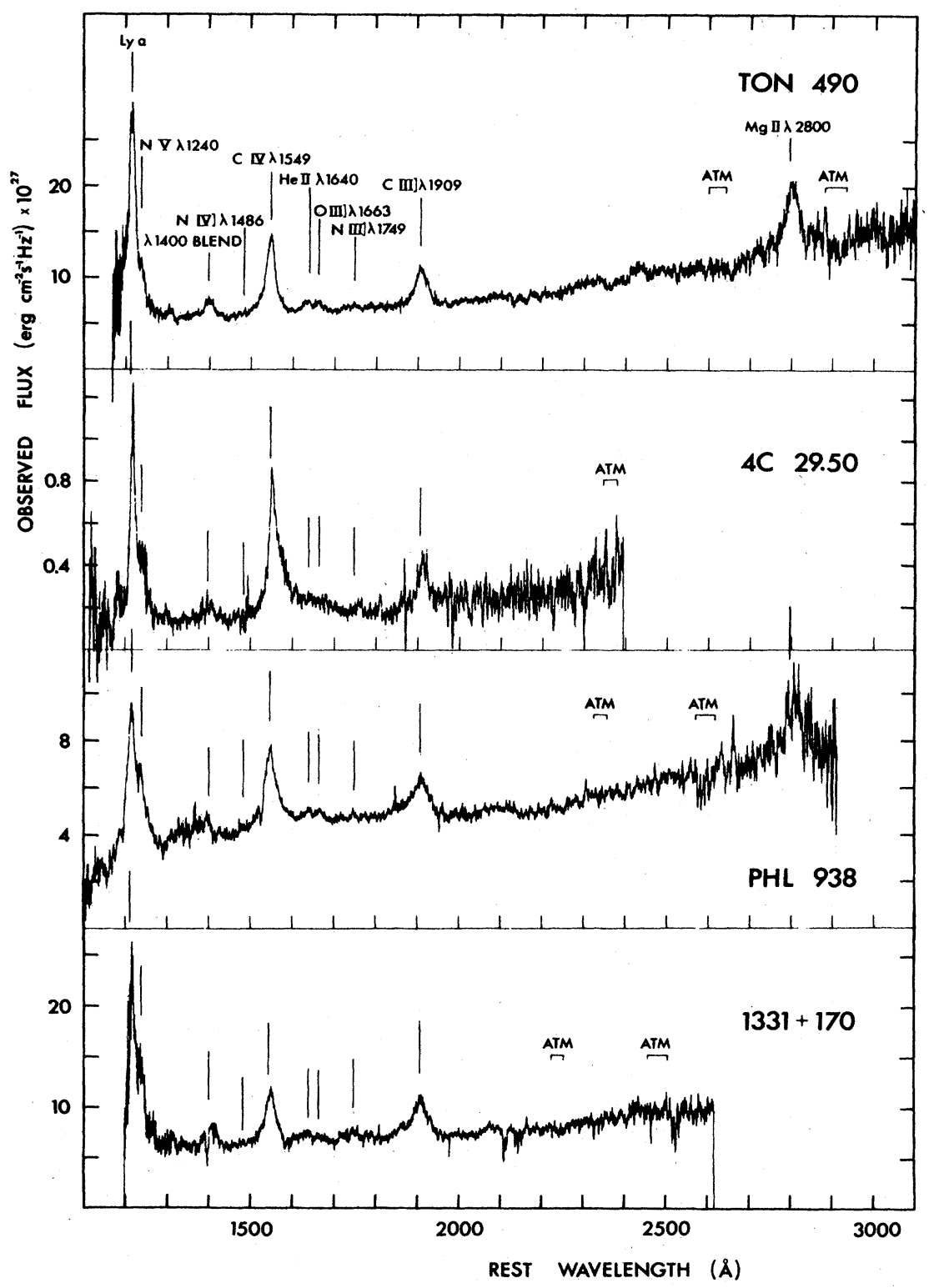

FIG. 4. Ultraviolet spectra of several high-red-shift quasars, from Baldwin and Netzer (1.978). (Note that the ordinates are fluxes per unit frequency, rather than fluxes per unit wavelength. Thus the emission features toward the left edge are underemphasized here.) Copyright The Astrophysical Journal.

sight velocities of the emitting region and the intervening absorbing gas) while another might be at $z_{a b}=0.80$ (in which case the indicated velocity difference would be comparable to the speed of light). There is a tendency for absorption systems to be concentrated near $z_{e m}$. Because the absorption lines are so narrow, some of them have easily observable fine structure (e.g.; the C IV doublet whose rest wavelengths are $1548,1551 \AA$ ), which sometimes leads to information about the gas and radiation densities in the absorbing gas clouds. Thus it is thought that these clouds are not dense enough or close enough to the quasars to be identified with the emissionline regions that we discuss in this review. There has been a long dispute, not yet settled, about whether the absorbing clouds have been ejected from the quasars or whether they are completely unrelated to the quasars. Assigning some clouds to each point of view may be possible. Here, we shall not discuss the narrow absorption lines, especially since in the past they have been reviewed more than the emission lines. See Perry, Burbidge, and Burbidge (1978) and many references therein, as well as Bahcall (1978).

A few peculiar quasars have broad absorption features that probably are related to their emission features; expanding shells or envelopes, with characteristic speeds exceeding $10^{4} \mathrm{~km} \mathrm{~s}^{-1}$, seem to be involved. See Lynds (1967), Burbidge (1970), Boksenberg, Carswell, Smith, and Whelan (1978), and Weedman, Lewis, and MacAlpine (1979).

Observational techniques and uncertainties, and methods used to find quasars, will also be neglected here. Some of these topics are discussed by Smith (1978). Another topic of interest is the distribution of quasars in red shift $z$; the large number of luminous high-red-shift quasars implies that such objects were far more common 10 or 15 billion years ago than they are today. If one could take samples of the past universe as a function of red shift $z$, the number of quas- 
ars per unit mass would increase somewhat like $(1+z)^{6}$, or perhaps like an exponential function of the "look-back time" to $z$. It also seems likely that quasars were typically more luminous in the past. See Schmidt $(1970,1972)$, Sramek and Weedman (1978), Turner (1979), and references cited therein.

\section{Topics included in this review}

In Sec. II, we shall review some of the physical processes and circumstances that are relevant to astronomical emission-line spectra; photoionization equilibria are particularly important. Section II is intended mainly for the reader who is unfamiliar with traditional nebular astrophysics, but we note also that there are important differences between classical nebular analyses and analyses of quasars' spectra.

In Sec. III, we describe the "photoionization models" that were developed, mostly between 1969 and 1976, to explain quasars' ultraviolet emission lines. These models give us some ideas of the relative elemental abundances in the emission regions - in particular, carbon, nitrogen, and oxygen appear to be about as abundant, relative to hydrogen, as in some nearby galactic nuclei. The photoionization models also indicate approximate values of the radiation-to-gas density ratio in the line-emitting regions, while giving rough limits on the gas density.

Section IV is a discussion of certain emission lines for which the emitting regions are optically thick, and the associated radiative-transfer problems. From the observed relative intensities of these lines, it is possible to guess characteristic radiation densities in the emission regions, and, therefore, the distances of these regions from the quasars' centers.

In Sec. V, we discuss how the likely existence of intervening solid "dust" grains can modify the preceding arguments. We favor models in which dust is significant, but in some of these models the quasars' luminosities are even more grandiose than previously suggested. The evidence is not yet conclusive.

Section VI is about some spectral features, not discussed in the preceding sections, which may convey useful information. Unfortunately, these features are poorly understood at present.

In Sec. VII we mention several ways in which the rest of our discussion may be wrong: these involve processes that we believe to be almost negligible, but it is possible to imagine loopholes in our reasoning. Any radically different, alternative type of model will probably have to be more complicated than our preferred models in order to achieve equal success in accounting for the observed line intensities.

Finally, Sec. VIII is a rather sketchy attempt to describe the ideas of many authors about gas dynamics and movements in the line-emitting regions. The profiles of emission lines are significant in this regard.

There are many pitfalls in this subject, whose nature is typified in three basic rules:

(i) Simplified models of quasars' emission regions are usually wrong, in the sense that many complicated effects can be shown to modify the physical parameters of the situation.

(ii) However, because observational data are limited and subject to a sort of "entropy," in practice one may as well use simplified models for many purposes anyhow! (But some experience is required in order to know how much simplification is allowable; for example, it is usually wrong to assume any particular uniform temperature.)

(iii) A specific explanation of just one or two spectral features-without considering the other features-is usually worthless.

\section{PHOTOIONIZED NEBULAE-A BRIEF OVERVIEW}

\section{A. The photoionization equilibrium: counting the photons}

Throughout our own Galaxy, hot, luminous stars radiate enough ultraviolet energy to photoionize their surrounding interstellar gas clouds-which then become "emission nebulae" or "H II regions." Physical conditions in these nebulae have become fairly well known since the work of Zanstra, Bowen, Strömgren, Menzel, Aller, and others, beginning before 1930. The processes involved in ordinary galactic nebulae have been reviewed by Aller (1956), Seaton $(1960,1972)$, Hummer and Seaton $(1963,1964)$, Spitzer $(1968,1978)$, Osterbrock (1974), and in a collection of papers edited by Menzel (1962). Here we shall present a sketch of these processes, with a different emphasis appropriate to a different type of photoionized nebula. Our idealized nebula will be associated with a quasarlike object rather than with a hot star; gas and radiation densities are higher than in a galactic nebula, while the energy distribution of the ionizing photons is quite different. For convenience, we shall first ignore some processes, and then acknowledge them later in the discussion. We shall not specify accurate values for most parameters of interest, because there are so many of them; references to such values will be cited in Sec. II.D.

Consider first an "optically thin" or transparent cloud of pure hydrogen gas, with density $n_{\mathrm{H}}$ of hydrogen atoms and ions, exposed to a monodirectional flux of ionizing photons. Molecular hydrogen is extremely scarce in the cloud because of the ultraviolet flux, so the ionization equilibrium involves only $\mathrm{H}^{0}$ and $\mathrm{H}^{+}$ions, with densities $n\left(\mathrm{H}^{0}\right)$ and $n\left(\mathrm{H}^{+}\right)$. Let $F(\varepsilon) d \varepsilon$ be the incident energy flux in photon energy interval $d \varepsilon$, approximated for our purposes by

$$
F(\varepsilon)=\left(\varepsilon / I_{\mathrm{H}}\right)^{-s} e^{-\varepsilon / \varepsilon_{0} F_{\mathrm{H}}} .
$$

Here $I_{\mathrm{H}}=13.6 \mathrm{eV}$, corresponding to a wavelength of $912 \AA$, is the ionization threshold of a ground-level hydrogen atom, often called the "Lyman limit" by astronomers. The flux parameter $F_{\mathrm{H}}$, the spectral index $s$, and the cutoff energy $\varepsilon_{0}$ are constant parameters. We shall suppose that $\varepsilon_{0} \gg I_{\mathrm{H}}$, and adopt $s=1$ as an exemplary value, which gives a flux $F_{\mathrm{H}}$ of ionizing photons $\left(\varepsilon>I_{\mathrm{H}}\right)$. The corresponding energy flux depends logarithmically upon $\varepsilon_{0}$, but since $x$-ray photons with $\varepsilon \gtrsim 1 \mathrm{keV}$ do not interact strongly with the gas, we can temporarily suppose that $\varepsilon_{0}$ is indefinitely large.

Most hydrogen atoms are in the ground level, so the only photoionization cross section that we need now is for the ground level of $\mathrm{H}^{\mathrm{o}}$; call this $\sigma_{\mathrm{H}}(\varepsilon)$. It can be calculated almost exactly, neglecting relativistic ef- 
fects (Bethe and Salpeter, 1957), and a good practical approximation is

$$
\begin{aligned}
& \sigma_{\mathrm{H}}(\varepsilon) \approx\left(6.3 \times 10^{-18} \mathrm{~cm}^{2}\right)\left(\varepsilon / I_{\mathrm{H}}\right)^{-2.8}, \\
& I_{\mathrm{H}}<\varepsilon \lesssim 100 \mathrm{eV} .
\end{aligned}
$$

The rate of photoionization events per unit volume is evidently

$$
n\left(\mathrm{H}^{0}\right) \int_{I_{\mathrm{H}}}^{\infty} \sigma_{\mathrm{H}}(\varepsilon) \frac{F(\varepsilon)}{\varepsilon} d \varepsilon \approx\left(\frac{10^{-17.2} \mathrm{~cm}^{2}}{2.8+s}\right) F_{\mathrm{H}} n\left(\mathrm{H}^{0}\right) .
$$

The inverse process is radiative recombination, whose rate per unit volume is $\alpha_{\mathrm{H}} n_{e} n\left(\mathrm{H}^{+}\right)$, where $\alpha_{\mathrm{H}}$ is a total "recombination coefficient" for hydrogen and the electron density $n_{e}$ is equal to $n\left(\mathrm{H}^{+}\right)$if the gas is pure hydrogen. This rate includes recombinations to all levels, quickly followed by cascading radiative decays to the ground level. The effective value of $\alpha_{\mathrm{H}}$ depends somewhat upon the distribution of free-electron energies; as we shall explain later, in cases of interest a typical free electron has a kinetic energy of the order of $2 \mathrm{eV}$ (corresponding to a kinetic temperature near $15000 \mathrm{~K}$ ), so that $\alpha_{\mathrm{H}}$ is of the order of $10^{-12.5} \mathrm{~cm}^{3} \mathrm{~s}^{-1}$ (Seaton, $1959,1960)$. The characteristic time to approach ionization equilibrium, $\left(\alpha_{\mathrm{H}} n_{e}\right)^{-1}$, will usually be quick enough for the $\mathrm{H}^{+} / \mathrm{H}^{0}$ ratio to be practically in equilibrium. Then,

$$
\frac{n\left(\mathrm{H}^{+}\right)}{n\left(\mathrm{H}^{0}\right)} \approx\left(\frac{10^{-4.7} \mathrm{~cm}^{-1} \mathrm{~s}}{2.8+s}\right) \frac{F_{\mathrm{H}}}{n_{e}} .
$$

It will be convenient to adopt an "ionization parameter" with velocitylike dimensions, $U_{\mathrm{H}}=F_{\mathrm{H}} / n_{e}$; then, for $s \approx 1$,

$$
\frac{n\left(\mathrm{H}^{+}\right)}{n\left(\mathrm{H}^{0}\right)} \approx\left(\frac{U_{\mathrm{H}}}{10^{5.3} \mathrm{~cm} \mathrm{~s}^{-1}}\right) \text {. }
$$

In typical photoionized nebulae, and probably in the gas associated with quasars, $U_{\mathrm{H}}$ generally happens to be larger than the speed of sound (which is slightly above $10^{6} \mathrm{~cm} \mathrm{~s}^{-1}$ ) but less than the speed of light. ${ }^{2}$ Then, according to Eq. (2.3b), the gas is mostly ionized if the gas cloud is "optically thin" or transparent to the ionizing radiation.

Now consider what happens if the cloud of gas is thick enough to absorb most of the ionizing photons. Suppose that the illuminated edge of the cloud is planar and that the ionizing photon flux is normally incident upon it. Suppose that the density $n_{\mathrm{H}}=n\left(\mathrm{H}^{0}\right)+n\left(\mathrm{H}^{+}\right)$is uniform within the cloud. At linear depth $x$ into a pure hydrogen cloud, the photon-energy-dependent "optical depth" is

$$
\tau(\varepsilon)=\sigma_{\mathrm{H}}(\varepsilon) \int_{0}^{x} n\left(\mathrm{H}^{0}\right) d x^{\prime}
$$

\footnotetext{
${ }^{2}$ Various parameters which are equivalent to $U_{\mathrm{H}}$ appear in papers on ionization near quasars. $U_{1}$, as used by Davidson $(1972,1973,1977 \mathrm{a}, \mathrm{b})$, Baldwin and Netzer (1978), and others, is practically the same as $U_{\mathrm{H}}$. The parameter $U_{1}$ used by Shields (1976), and $\Gamma$ used by Mathews (1975) are essentially $U_{\mathrm{H}} / c$. Other authors have used more complicated parameters, sometimes denoted $\zeta, \xi$, or $y$. The effective pressures or kinetic energy densities of ionizing radiation and of gas are roughly equal, for $s \approx 1$, if $U_{\mathrm{H}} \approx 0.1 c \approx 10^{9.5} \mathrm{~cm} \mathrm{~s}^{-1}$.
}

the local photon flux is reduced to $F(\varepsilon) e^{-\tau(\varepsilon)}$, and the local ionization ratio $n\left(\mathrm{H}^{+}\right) / n\left(\mathrm{H}^{0}\right)$ is decreased accordingly. At some depth $x=x_{\mathrm{H}}$, there is an "ionization front" or transition zone where $n\left(\mathrm{H}^{0}\right)$ becomes larger than $n\left(\mathrm{H}^{+}\right)$. One can integrate numerically to find $n\left(\mathrm{H}^{\circ}\right)$, $n\left(\mathrm{H}^{+}\right)$, and $\tau(\varepsilon)$ as functions of $x$; but without actually doing this, we can easily guess the approximate ionized thickness $x_{\mathrm{H}}$, as follows. For reasonably large $U_{\mathrm{H}}$, $n\left(\mathrm{H}^{+}\right) / n\left(\mathrm{H}^{0}\right) \gg 1$ throughout most of the ionized region, so $n_{e}=n\left(\mathrm{H}^{+}\right) \approx n_{\mathrm{H}}$ there. The total column rate of recombinations per unit illuminated area is thus

$$
\int_{0}^{x_{\mathrm{H}}} \alpha_{\mathrm{H}} n_{e} n\left(\mathrm{H}^{+}\right) d x^{\prime} \approx \alpha_{\mathrm{H}} n_{\mathrm{H}}^{2} x_{\mathrm{H}}
$$

This must equal the total flux of absorbed ionizing photons, which is of the order of $F_{\mathrm{H}}$; therefore $x_{\mathrm{H}}$ $\approx F_{\mathrm{H}} / \alpha_{\mathrm{H}} n_{\mathrm{H}}^{2}$. The material thickness of the ionized region is proportional to $U_{\mathrm{H}}$ :

$$
n_{\mathrm{H}} x_{\mathrm{H}} \approx n_{e} x_{\mathrm{H}} \approx U_{\mathrm{H}} / \alpha_{\mathrm{H}} \approx\left(10^{23} \mathrm{~cm}^{-2}\right) U_{\mathrm{H}} / c .
$$

(If the gas were initially un-ionized and the flux $F_{\mathrm{H}}$ started instantaneously, then the time required to establish the equilibrium ionized region would be several times the recombination time $\left(\alpha_{\mathrm{H}} n_{\mathrm{H}}\right)^{-1} \approx x_{\mathrm{H}} / U_{\mathrm{H}}$. Hence $U_{\mathrm{H}}$ may be interpreted as a "speed of adjustment" to flux variations. We also note that scattering of photons by free electrons may be neglected if $n_{e} x_{\mathrm{H}} \ll 10^{24} \mathrm{~cm}^{-2}$, i.e., if $U_{\mathrm{H}} \ll 3 \times 10^{11} \mathrm{~cm} \mathrm{~s}^{-1}$.)

Let $\Delta x_{\mathrm{H}}$ denote the thickness of the ionization front, say, the distance between points where the gas is $>80 \%$ ionized and $<20 \%$ ionized. Then $\Delta x_{\mathrm{H}} \approx K / \sigma_{\mathrm{H}} n_{\mathrm{H}}$, where $\sigma_{\mathrm{H}}$ is the threshold cross section and $K$ is of the order of 20 for realistic cases (wherein high-energy photons are absorbed by ions other than hydrogen) and larger for the pure-hydrogen case. Using the smaller value of $K$,

$$
\frac{\Delta x_{\mathrm{H}}}{x_{\mathrm{H}}} \approx\left(\frac{10^{6} \mathrm{~cm} \mathrm{~s}^{-1}}{U_{\mathrm{H}}}\right) .
$$

This means that the ionization front is well defined for $U_{\mathrm{H}} \gg 10^{6} \mathrm{~cm} \mathrm{~s}^{-1}$ and becomes relatively narrower and more distinct as $U_{\mathrm{H}}$ is increased.

A real cloud of gas will contain other elements besides hydrogen. In most of our galaxy, the heliumhydrogen ratio is about $n_{\mathrm{He}} / n_{\mathrm{H}} \approx 0.1$, while heavier ions-chiefly carbon, nitrogen, oxygen, and neon-have abundances $n_{z} / n_{\mathrm{H}}<10^{-3}$; a major aim in the analysis of quasars' spectra is to determine whether the material associated with quasars has a similar composition. Photoionization equilibria for these elements-e.g., $n\left(\mathrm{He}^{++}\right) / n\left(\mathrm{He}^{+}\right) / n\left(\mathrm{He}^{0}\right)$ - can be estimated by a nearly straightforward extension of the method used for hydrogen. Multiply charged ions have higher ionization thresholds, smaller threshold photoionization cross sections, and larger recombination coefficients than hydrogen; so for a given value of $U_{\mathrm{H}}$, their successive ionization ratios are smaller than that given for hydrogen by Eq. (2.3b). For example, with spectral index $s \approx 1$,

$$
\frac{n\left(\mathrm{He}^{++}\right)}{n\left(\mathrm{He}^{+}\right)} \approx\left(\frac{\dot{U}_{\mathrm{H}}}{10^{7.2} \mathrm{~cm} \mathrm{~s}^{-1}}\right) \text {. }
$$

In an optically thick cloud with a "conventional" com- 
position, most of the ionizing photons are absorbed by hydrogen and helium, while the scarcer, heavier ions have much less effect on the positional dependence of the ultraviolet flux. Three important ionization fronts occur, involving $\mathrm{He}^{++} / \mathrm{He}^{+}, \mathrm{He}^{+} / \mathrm{He}^{0}$, and $\mathrm{H}^{+} / \mathrm{H}^{0}$, with relevant ionization thresholds $54.4,24.6$, and $13.6 \mathrm{eV}$, respectively. Most analyses related to quasars find three important ionization zones, as follows:

(a) Near the illuminated boundary of the gas cloud, photons of all energies are found. If $U_{\mathrm{H}}$ is large enough, helium is mostly $\mathrm{He}^{++}$and hydrogen is mostly $\mathrm{H}^{+}$. Most of the photons with $\varepsilon>54.4 \mathrm{eV}$ are absorbed in maintaining the $\mathrm{He}^{++} / \mathrm{He}^{+}$equilibrium in this zone. The relative thickness of the $\mathrm{He}^{++} / \mathrm{He}^{+}$ionization front is larger than that given by Eq. (2.6).

(b) Beyond the $\mathrm{He}^{++} / \mathrm{He}^{+}$ionization front lies a zone where most of the photons (except perhaps some highly penetrating $\mathrm{x}$-ray photons) have energies below 54.4 $\mathrm{eV}$, and where $\mathrm{He}^{+}$and $\mathrm{H}^{+}$predominate. In principle, this zone can be bounded by either the $\mathrm{H}^{+} / \mathrm{H}^{0}$ or the $\mathrm{He}^{+} / \mathrm{He}^{0}$ ionization front, depending upon the incident ionizing spectrum and the helium/hydrogen abundance ratio. But for likely ranges of parameters, the positions of these two ionization fronts are interdependent and tend to occur almost together. ${ }^{3}$

(c) The third zone contains mostly $\mathrm{H}^{0}$ and $\mathrm{He}^{0}$, with photon energies mostly below $13.6 \mathrm{eV}$. However, $\mathrm{x}$-ray photons, with $\varepsilon \gtrsim 200 \mathrm{eV}$, may penetrate into this zone to cause some ionization.

In such a neatly stratified arrangement of zones, the heavier elements are ionized according to their ionization thresholds. Carbon is a good example. The thresholds of $\mathrm{C}^{0}, \mathrm{C}^{+}, \mathrm{C}^{++}, \mathrm{C}^{3+}, \mathrm{C}^{4+}$, and $\mathrm{C}^{5^{+}}$are 11.3, $24.4,47.9,64.5,392$, and $490 \mathrm{eV}$. The last two thresholds are so high that the $\mathrm{C}^{6+} / \mathrm{C}^{5+} / \mathrm{C}^{4+}$ equilibrium depends upon whether there is an $\mathrm{x}$-ray "cutoff" in the power law spectrum; and also, large values of $U_{\mathrm{H}}$ would be required to make $\mathrm{C}^{6+}$ or $\mathrm{C}^{5+}$ predominate. Therefore in the highly ionized zone (a), if $U_{\mathrm{H}}$ is moderately large, carbon may be either $\mathrm{C}^{4+}, \mathrm{C}^{5+}$, or $\mathrm{C}^{6+}$. In the $\mathrm{He}^{+}$zone (b), carbon would be mostly $\mathrm{C}^{3+}$ (because photons to ionize further are lacking there), and in zone (c), carbon would be $\mathrm{C}^{+}$. However, if $U_{\mathrm{H}} \lesssim 10^{9}$ $\mathrm{cm} \mathrm{s}^{-1}$, it is found that significant equilibrium concentrations of $\mathrm{C}^{++}$and $\mathrm{C}^{3+}$ also occur in zone (a) while a significant amount of $\mathrm{C}^{++}$occurs in zone (b).

We shall now mention some complications, which in most cases do not lead to qualitative changes. First, it is evident that some diffuse secondary ionizing radiation must be produced within the ionized gas. For example, when a typical $2-\mathrm{eV}$ free electron radiatively recombines with a proton in such a way that a groundlevel $\mathrm{H}^{0}$ atom results directly, then a $15.6-\mathrm{eV}$ ionizing photon is emitted. Unless the gas cloud is optically thin, this photon is likely to be reabsorbed to cause another ionization. In some cases, one can crudely allow for this by pretending that such a secondary ioniz-

\footnotetext{
${ }^{3}$ In many galactic nebulae, the central stars have ionizing spectra which decrease steeply above $\varepsilon=13.6 \mathrm{eV}$; such nebulae have sizable regions where $\mathrm{H}^{+}$and $\mathrm{He}^{0}$ predominate together. But this is not the case for most photoionization models related to quasars.
}

ing photon is reabsorbed "on the spot," so that, in effect, the recombination event never occurred. Thus, instead of using the total recombination coefficient, in the "on the spot approximation" one allows recombinations only to the excited levels of hydrogen, which produce only nonionizing photons. This reduces the effective value of $\alpha_{H}$ by as much as $40 \%$. Similar approximations can be used for some helium recombinations but are usually unnecessary for heavier elements. Where more precise calculations are desired, it is necessary to solve the photon-energy-dependent radiative transfer problem by an iterative method. However, such a proper solution is geometry dependent.

For large values of $U_{\mathrm{H}}$ and photons in the x-ray energy range, ions heavier than helium are important for determining the radiative transfer, even if they are much less abundant than helium; but the relevant ionizing fluxes for $E \gg 100 \mathrm{eV}$ are quite uncertain in any case.

Collisional ionization by free electrons is usually of secondary importance in photoionized regions. However, under conditions discussed in Sec. IV.C below, collisional ionization from the $n=2$ level of hydrogen becomes important near the $\mathrm{H}^{+} / \mathrm{H}^{0}$ ionization front. The inverse process, three-body nonradiative recombination, is negligible if $n_{e} \ll 10^{11} \mathrm{~cm}^{-3}$.

Dielectronic recombination, wherein the recombining electron disposes of its initial energy by raising a bound electron to an excited level, is important for some ions, and is rather sensitive to the initial electron energy.

Various charge-exchange reactions are also important. A notable example is $\mathrm{C}^{3+}+\mathrm{H}^{0} \rightarrow \mathrm{C}^{++}+\mathrm{H}^{+}$, which is important in models with $U_{\mathrm{H}} \lesssim 10^{8} \mathrm{~cm} \mathrm{~s}^{-1}$. (The inverse process is negligible, because of Coulomb repulsion between $\mathrm{C}^{++}$and $\mathrm{H}^{+}$ions.)

Photoionization cross sections are not easy to calculate for multielectron ions; and unlike the case for most galactic nebulae, photoionization from inner shells of electrons must be taken into account if the ionizing spectrum is quasarlike. When an inner-shell electron has been removed, the resulting ion is in an excited state. If it decays radiatively, a secondary ionizing photon may be produced. In the case of a removed $K$-shell electron, ionic readjustment often involves the Auger effect, wherein one or more additional electrons are ejected. Moreover, electrons which have been freed by $x$-ray photons may be so energetic that they cause secondary ionizations and excitations before being "thermalized" as discussed in the next section.

Quantitative details about most of these processes may be found in the references cited in Sec. II.D below.

\section{B. Thermal equilibrium: counting the ergs}

Having described the equilibrium between ionization and recombination events, we now consider the equilibrium between energy gains and losses in the ionized gas. A very convenient and accurate approximation can be introduced immediately: In most cases, the distributions of kinetic energies of ions and free electrons are practically Maxwellian, with just one kinetic temperature $T$ applying to all ions and electrons. This is true because of the effectiveness of elastic collisions 
between charged particles in redistributing kinetic energies; see Spitzer (1962). We must emphasize, very strongly, that the temperature $T$ characterizes only the kinetic energies; the radiation field, the ionization ratios, and the populations of ionic internal energy levels are often very different from ordinary thermal distributions. ${ }^{4}$

Consider some locale within a pure-hydrogen photoionized region. When a photon of energy $\varepsilon$ is absorbed in a photoionization event, the initial kinetic energy of the freed electron is nearly $\left(\varepsilon-I_{\mathrm{H}}\right)$. Elastic collisions very quickly spread this energy among the ions and electrons, the net result being a kinetic energy "heating" contribution of $\left(\varepsilon-I_{\mathrm{H}}\right)$. The total local heating rate can obviously be calculated from the local values of $n\left(\mathrm{H}^{0}\right)$ and $F(\varepsilon)$ :

(heating per unit volume) $=$

$$
n\left(\mathrm{H}^{0}\right) \int_{r_{\mathrm{H}}}^{\infty}\left(\varepsilon-I_{\mathrm{H}}\right) \sigma_{\mathrm{H}}(\varepsilon) \frac{F(\varepsilon)}{\varepsilon} d \varepsilon .
$$

For the moment, we shall neglect other heating processes: adiabatic compression by shock waves, inverse-bremsstrahlung absorption of radio-frequency waves, excitation by energetic charged particles, viscous dissipation of turbulent motions, conduction of heat from adjoining hotter gas, magnetic field line reconnection, etc. Most of these are probably unimportant in a quasar's emission-line regions.

Several types of "cooling" processes remove kinetic energy from the gas by converting it to photons. Most obviously, radiative recombination removes electrons with various energies. Integrating the product of the energy-dependent recombination rate with the Maxwellian distribution, one finds that the average recombining electron has an initial kinetic energy close to $k T$. This energy, plus some binding energy, becomes a photon. Rather crudely,

(recombination cooling per unit volume) $\approx$

$$
\alpha_{\mathrm{H}} n_{e} n\left(\mathrm{H}^{+}\right) k T,
$$

where the recombination coefficient $\alpha_{\mathrm{H}}$ is itself a function of $T$. (In fact, $\alpha_{\mathrm{H}} \sim T^{-0.7}$ for temperatures of interest.) More precise values of this cooling rate have been calculated by Seaton $(1959,1960)$. The bremsstrahlung process causes additional cooling, wherein electron-ion Coulomb collisions convert some kinetic energy into radiation. This is very closely related to radiative recombination; recombinations are "freebound" transitions and bremsstrahlung events are "free-free" transitions by the electrons. Consequently, the bremsstrahlung cooling rate is comparable to the recombination cooling rate. Equation (2.8) would crudely represent the total of recombination and bremsstrahlung cooling if $3 k T / 2$ were used instead of $k T$. For temperatures of interest, most bremsstrahlung and recombination photons have $\varepsilon<I_{\mathrm{H}}$ and hence can escape

\footnotetext{
${ }^{4}$ The the rmal distribution of electrons actually extends downward into the closely spaced uppermost bound states of ions, e.g., levels $n>10$ for hydrogen; the free electrons' zero-energy level is not perfectly straightforward to define. But for most nebular purposes, this fact is of minor importance.
}

from the gas with little difficulty; but as mentioned in the preceding section, some ionizing photons are also produced, whose subsequent transfer must be considered in any elaborate calculation.

Another cooling process, in a pure-hydrogen nebula, involves inelastic collisions of free electrons with hydrogen atoms. Such collisions convert kinetic energy into excitation energy, usually transfering atoms from the $n=1$ ground level to the $n=2$ or $n=3$ levels; the atoms then decay radiatively back to $n=1$, so that the net result is conversion of kinetic energy to photons. As mentioned earlier, typical electron energies $3 k T / 2$ will turn out to be around $2 \mathrm{eV}$ in a real nebula, while excitation energies of ions are usually much larger (e.g., $10 \mathrm{eV}$ from $n=1$ to $n=2$ in $\mathrm{H}^{0}$ ). Therefore only the electrons in the high-energy "tail" of the Maxwellian distribution can cause excitations. Nevertheless, the excitation process inherently has such large cross sections that its overall rate per unit volume can exceed the recombination and bremsstrahlung cooling rates. Because a factor $\exp \left(-E_{i j} / k T\right)$ is involved, with $E_{i j} \gtrless 2 k T$, the collisional-excitation cooling rate is very sensitive to temperature and therefore is a fairly good thermostat.

The above cooling processes all involve electron-ion interactions. Ion-ion interactions (meaning $\mathrm{H}^{0}-\mathrm{H}^{+}$or $\mathrm{H}^{0}-\mathrm{H}^{0}$ for hydrogen) are negligible for optical and ultraviolet transitions, because the collision times exceed the ions' internal natural periods $\hbar / E_{i j}$. Electron-electron collisions are very elastic because they involve no electric dipole moments.

Having assembled the cooling and heating rates as functions of temperature in our fictitious pure-hydrogen nebula, we can find the equilibrium temperature where heating is balanced by cooling. The ionization and thermal equilibria are interdependent, because the effective recombination rate $\alpha_{\mathrm{H}}$ is temperature dependent while the heating and cooling rates depend upon $n\left(\mathrm{H}^{+}\right) /$ $n\left(\mathrm{H}^{0}\right)$. Therefore an iterative procedure must be used to find a self-consistent solution; but this does not cause much difficulty in practice. If the cooling is mainly due to recombination and bremsstrahlung (as in pure hydrogen gas with large $U_{\mathrm{H}}$ ), then the equilibrium value of $k T$ is roughly the same as the average value of $\left(\varepsilon-I_{\mathrm{H}}\right)$ for the absorbed photons. But if collisional excitation dominates the cooling rate (as in a low $-U_{\mathrm{H}}$ hydrogen case, or in the realistic case mentioned below), then the temperature is lower.

What if the gas has a realistic composition, rather than being pure hydrogen? The primary changes in the heating rate are obvious: photoionization of each ion species must be taken into account, including inner shells. Recombination and bremsstrahlung cooling rates are also augmented slightly. In some cases, most of the heating as well as the recombination and bremsstrahlung cooling involve just hydrogen and helium. The less abundant heavier ions are very important, though, because some of them are efficient collisionalexcitation coolants. For example, imagine a rather typical ionized region, where the most abundant elements are largely in the forms of $\mathrm{H}^{+}, \mathrm{C}^{3+}, \mathrm{O}^{2+}$, etc. Consider collisional excitations of the various ion species. Hydrogen is the most abundant element, but 
only a tiny fraction if it is excitable $\mathrm{H}^{0}$. As for helium, neither $\mathrm{He}^{+}$nor $\mathrm{He}^{0}$ can be excited with an energy of less than $21 \mathrm{eV}$; this is so high as to be negligible if $k T<4 \mathrm{eV}$. But $\mathrm{C}^{3+}$ has an excited level at $8 \mathrm{eV}$, and because of the ionization, $n\left(\mathrm{C}^{3+}\right)$ may be comparable to $n\left(\mathrm{H}^{0}\right)$. The ion $\mathrm{O}^{2+}$ and other multielectron ions also have excited levels of interest. Therefore collisional excitation of certain heavy ions may easily dominate the cooling. This is the main reason why rather "low" temperatures, of the order of $T \approx 15000 \mathrm{~K}$ or $k T \approx 1.3 \mathrm{eV}$, are found in photoionization calculations with roughly "conventional" heavy-element abundances-even if most photoionization events give initial kinetic energies of $10 \mathrm{eV}$ or more to the freed electrons. It is worth remarking that a real photoionized gas is almost always thermally stable - the cooling/heating ratio rises quickly with temperature.

We shall now be more specific about collisional excitation rates. Let $\mathrm{Z}^{2+}$ denote an ion species, two of whose energy levels are $i$ and $j$. Suppose that $j$ is above $i$, the energy difference being $E_{i j}$. The levels' statistical weights (numbers of states) are $\omega_{i}$ and $\omega_{j}$. If an ion is in level $i$, then inelastic collisions by free electrons will cause transitions to level $j$, at rate $C_{i j}$. It has become customary to employ a dimensionless "collision strength" $\Omega_{i j}$ to specify the rate as a function of temperature:

$$
\begin{aligned}
C_{i j} & =\frac{2 a_{0}^{2} c}{137}\left(\frac{\pi I_{\mathrm{H}}}{k T}\right)^{1 / 2}\left(\frac{\Omega_{i j}}{\omega_{i}}\right) n_{e} e^{-E_{i j} / k T} \\
& =\left(10^{-7.06} \mathrm{~cm}^{3} \mathrm{~s}^{-1}\right)\left(\frac{10^{4} \mathrm{~K}}{T}\right)^{1 / 2}\left(\frac{\Omega_{i j}}{\omega_{i}}\right) n_{e} e^{-E_{i j} / k T} .
\end{aligned}
$$

The inverse rate for an ion in level $j$ to be collisionally deexcited to level $i$, causing a free electron to gain energy, is

$$
C_{j i}=\left(10^{-7.06} \mathrm{~cm}^{3} \mathrm{~s}^{-1}\right) \cdot\left(\frac{10^{4} \mathrm{~K}}{T}\right)^{1 / 2}\left(\frac{\Omega_{i j}}{\omega_{j}}\right) n_{e} .
$$

The effective collision strength $\Omega_{i j}$ is really an integral involving cross sections, electron velocities, and the Maxwellian distribution; it has the virtue of being approximately independent of temperature for most transitions and temperatures of interest. Furthermore, $\Omega_{i j} / \omega_{i}$ and $\Omega_{i j} / \omega_{j}$ are often of the order of unity for optical and ultraviolet energy transitions. For a better definition of $\Omega_{i j}$, see Seaton $(1958,1960)$ or Osterbrock (1974); but note that our $\Omega_{i j}$ is assumed to be averaged appropriately over electron velocities.

Radiative transitions between levels $i$ and $j$ can also occur. In the present context, incident photon fluxes will usually be low enough so that radiative excitation and stimulated emission rates are much less than $C_{i j}$ and $C_{j i}$; therefore, consider only spontaneous radiative decay from $j$ to $i$, with rate $A_{j i}$. Let $i$ be the ground level of ion $Z^{2+}$. For simplicity we shall pretend that $i$ and $j$ are the only levels of interest. The equilibrium ratio of densities of ions in the two levels is evidently $n_{j} / n_{i}=C_{i j} /\left(A_{j i}+C_{j i}\right)$, and the effective cooling rate, involving emission of photons, is $E_{i j} A_{j i} n_{j}$. In a highdensity, "collisionally deexcited" case, where $C_{j i}$ $\gg A_{j i}$, the population ratio $n_{j} / n_{i}$ is close to the simple thermal ratio $C_{i j} / C_{j i}=\left(\omega_{j} / \omega_{i}\right) e^{-E_{i j} / k T}$, which is small for most ions of interest in photoionized regions because usually $E_{i j} \gtrless 2$ or $3 \mathrm{eV}$ and $k T \approx 2 \mathrm{eV}$. In a lowdensity, "radiatively deexcited" case where $A_{j i} \gg C_{j i}$, $n_{j} / n_{i}$ becomes very small. As a first approximation, therefore, we can neglect excited ions when we calculate photoionization rates; we can similarly neglect cooling due to the further excitation of excited ions (i.e., collisional excitation from level $j$ to a higher level).

A proper calculation is often more complicated than the two-level approximation described above, but the qualitative result remains valid. Sometimes level $j$ may decay to an intermediate third level; or different states in level $j$ may have very different radiative decay rates. Then two or more equilibrium equations must be solved simultaneously to find the level populations. Excited levels may be populated by recombination events as well as collisional excitations. Another complication arises if the emitted photons of energy $E_{i j}$ are reabsorbed by ions in level $i$. Then such photons may escape from the gas only after many resonance "scattering" (actually absorption and reemission) events, and collisional deexcitation becomes more important than the ratio $C_{j i} / A_{j i}$ might suggest. The effects of resonance scattering are discussed in Sec. IV below.

\section{The emergent spectrum}

Nebular emission with a continuous spectrum, resulting from recombination and bremsstrahlung, is observationally difficult to separate from the basic continuous spectrum of the quasar itself; so we are more interested in nebular spectral lines, which are easier to observe. The most important elements for producing these lines are hydrogen, helium, carbon, nitrogen, oxygen, magnesium, and perhaps silicon and iron.

Observable emission lines in the spectrum of a nebula result, of course, from radiative decays of ions in various excited levels. Some excited levels are populated by recombination events; the resulting emission intensities convey information about total recombination rates. Other levels are populated chiefly by collisional excitation; the resulting emission lines give information about cooling rates. In ordinary nebulae, hydrogen and helium produce mainly recombination lines while heavier ions produce collisionally excited lines. (One intense exception is the Lyman-alpha line of hydrogen, for which collisional excitation can be comparable to recombination.) Two differences between recombination lines and collisionally excited lines should be borne in mind: (1) the emissivity per unit volume for a recombination line of ion $\mathrm{Z}^{z+}$ is proportional to $n\left(\mathrm{Z}^{+z+1}\right)$, while a collisionally excited emissivity is proportional to $n\left(Z^{z+}\right)$; and (2) the intensities of recombination lines are not strongly sensitive to the ratios $E_{i j} / k T$, while factors $e^{-E_{i j} / k T}$ are involved in collisional excitation.

Several classes of transitions may be distinguished. Permitted transitions satisfy the electric-dipole radiative selection rules. Two important examples are $\mathrm{H}^{0}$ $1 s-2 p\left(E_{i j}=10.2 \mathrm{eV}\right)$ and $C^{3+} 2 s-2 p(8.0 \mathrm{eV})$. The former is the Lyman-alpha (" $L \alpha$ ") line at a wavelength of 1215 $\AA$, or “H I $\lambda 1215$ "; the latter we shall call "C IV $\lambda 1549$. ." 
(Strictly speaking, $\lambda 1549$ is a doublet, $\lambda \lambda 1548,1551$. Permitted optical or UV transitions typically have $A_{j i}$ $\sim 10^{8} \mathrm{~s}^{-1}$ and $\left(\Omega_{i j} / \omega_{j}\right) \sim 1$, so that $C_{j i}=A_{j i}$ at densities of the order of $n_{e} \sim 10^{15} \mathrm{~cm}^{-3}$. However, because permitted photons may undergo many resonance scattering events before escaping from the gas, and each such event gives another chance for collisional deexcitation, collisional deexcitation can be important at densities as low as $10^{11}$ or even $10^{10} \mathrm{~cm}^{-3}$ (see Sec. IV below).

Intercombination or semiforbidden transitions involve spin changes. An example is $\mathrm{C}^{++} 2 s^{2}{ }^{1} S-2 s 2 p^{2} P^{0}\left(E_{i j}\right.$ $=6.5 \mathrm{eV}$ ), which gives rise to the C III] $\lambda 1909$ line. (The single square right-hand bracket indicates an intercombination line.) For these transitions, $A_{j i}$ can be of the order of $100 \mathrm{~s}^{-1}$ while $\left(\Omega_{i j} / \omega_{i}\right)$ is almost as large as for permitted transitions. Therefore collisional deexcitation becomes important at densities of the order of $n_{e} \approx 10^{9}$ or $10^{10} \mathrm{~cm}^{-3}$.

Forbidden transitions are electric quadrupole or magnetic dipole transitions, sometimes with spin changes. For example, nebular astronomers often study the ${ }^{3} P-{ }^{1} D$ transition $\left(E_{i j}=2.5 \mathrm{eV}\right)$ in the ground $\left(2 p^{2}\right)$ configuration of $\mathrm{O}^{++}$; the resulting emission lines are [OIII] $\lambda \lambda 5007,4959$, where the square brackets indicate forbiddenness. Forbidden lines are very important in ordinary galactic nebulae because of their modest excitation energies, but collisional deexcitation makes them less important in quasars. For example, [OIII] $\lambda 5007$ has $A_{j i} \approx 0.02 \mathrm{~s}^{-1}$ and $\left(\Omega_{i j} / \omega_{i}\right) \approx 0.5$, so collisional deexcitation of this transition sets in between $n_{e}=10^{5}$ and $10^{6} \mathrm{~cm}^{-3}$. (Most galactic nebulae have densities $n_{e} \ll 10^{6} \mathrm{~cm}^{-3}$; quasars have denser gas.)

Many forbidden lines have wavelengths between 3000 and $7000 \AA$, which can penetrate the Earth's atmosphere. Most important permitted and semiforbidden lines (excepting hydrogen and helium recombination lines) are between 1000 and $3000 \AA$ and can be observed from the Earth's surface only in the highly red-shifted spectra of distant quasars. This is one reason for the great difference between classical nebular analysis and the study of quasars' spectra. Astronomers have only recently begun to use instruments above the terrestrial atmosphere to observe the ultraviolet spectra of ordinary nebulae.

Fine-structure transitions, usually at infrared energies for ions like carbon or oxygen, can be excited or deexcited by ion-ion as well as electron-ion collisions. Typical fine-structure excitations are collisionally deexcited at lower densities than the visual-wavelength forbidden lines.

One should be aware of the possibility of two-photon radiative processes. The most important example occurs in hydrogen at low densities. There, a hydrogen atom in the $2 s$ level may decay, via a virtual intermediate state, to the $1 \mathrm{~s}$ level, emitting two photons whose total energy is $10.2 \mathrm{eV}$; the rate is $8 \mathrm{~s}^{-1}$ and the emitted spectrum is continuous. If $n_{e} \gg 10^{4} \mathrm{~cm}^{-3}$, this process is negligible because collisionally induced $2 s-2 p$ transitions are then faster.

Finally, we note that optical depth effects can alter the relative emergent intensities of some emission lines. The best-known example is the case of hydrogen. Almost any photoionized nebula is optically very thick in each spectral line of the Lyman series (whose lower level is the $n=1$ ground level); but since relatively few of the hydrogen atoms are in the $n \geqslant 2$ levels, it is possible for a nebula to be transparent to any of the Balmer, Paschen, etc., lines. Suppose, for example, that a recombination event has just produced a hydrogen atom in the $n=3$ level. It can decay either through $n$ $=2$, emitting a Balmer-alpha (" $H \alpha$ ") and then a Lymanalpha (" $L \alpha$ ") photon, or directly to $n=1$, emitting a Lyman-beta (" $L \beta$ ") photon. In the former case, the $H \alpha$ photon may escape easily and the $L \alpha$ photon emerges after many resonance scattering events; but in the latter case, the $L \beta$ photon will almost certainly be reabsorbed, exciting another hydrogen atom to the $n=3$ level and giving another chance for decay by either route. Obviously, $H \alpha$ and $L \alpha$ emission are enhanced while $L \beta$ is suppressed in this situation. It is sometimes legitimate to suppose that all Lyman-series emission is thus degraded into $L \alpha$ plus Balmer, Paschen, etc., lines (this is referred to in the literature as the "Case B" approximation). More complicated cases will be mentioned below in Sec. IV; we shall also see in Sec. V how the presence of continuum-absorbing material, such as dust grains, can modify an emergent nebular spectrum.

\section{Where to find ionic data}

In this section we merely cite references where quantitative values are given for the ionic processes mentioned above. For the ions of interest, most available parameters have been calculated rather than measured; some reviews of the methods have been given by Seaton (1958), Moiseiwitsch and Smith (1968), Bely and Van Regemorter (1970), Burke (1972), and others. Osterbrock (1974) has provided many very handy tables of useful quantities; but serious typographical errors occur in some of these tables, so cross-checking is always advisable.

Karzas and Latter (1961) calculated many values associated with radiative transitions by an electron in a Coulomb field. These data are relevant to bremsstrahlung, radiative recombination, and photoionization involving hydrogenic ions.

The photoionization cross section of atomic hydrogen has been described by Menzel and Pekeris (1935), Bethe and Salpeter (1957), and Burgess (1964). This can be modified to apply to other hydrogenic (i.e., oneelectron) ions. For the photoionization cross section of $\mathrm{He}^{0}$, see Bell and Kingston (1967), Brown (1971), and Marr and West (1976). Note that many of the cross sections and interaction coefficients of interest here have complicated resonance features, which are usually blurred or averaged out for practical applications.

Cross sections for photoionization of outer shells of ions of $\mathrm{C}, \mathrm{N}, \mathrm{O}$, and Ne are given by Henry (1970), Hidalgo (1968), and Sakhibullin and Willis (1978); miscellaneous heavier ions have been similarly treated by Conneely et al. (1970), Chapman and Henry (1971, 1972), Black et al. (1972), Shevelko (1974), Daum and Kelly (1976), Combet Farnoux and Lamoureux (1976), and others. Regarding photoionization of inner electron shells, see Daltabuit and Cox (1972), Weisheit (1974), and Weisheit and Collins (1976). The "Stobbe formulae" are useful for these (Bearden, 1966). Victor and Con- 
stantinides (1979) have discussed "double photoionization," in particular $\mathrm{O}^{\circ} \rightarrow \mathrm{O}^{++}+2 e^{-}$, which has not been included in quasar-related calculations. The Auger effect is discussed by MacAlpine (1974), by Bergeron and Collin-Souffrin (1974), and by Weisheit (1974). Dalgarno and McCray (1972) and Shull (1979) have discussed xray heating in general.

Radiative recombination coefficients for hydrogen (and by extension, other hydrogenic ions), as functions of temperature, have been calculated by Seaton (1959), Bates and Dalgarno (1962), Boardman (1964), Glasco and Zirin (1964), and Burgess (1964). Similar values for helium have been given by Burgess and Seaton (1960) and by Robbins $(1968,1970)$. For discussions of hydrogen and helium emission lines and level populations resulting from recombinations in ordinary nebulae, see Brocklehurst (1970, 1971, 1972), Giles (1977), and Seaton (1978). Radiative recombination coefficients for more complex ions have been estimated by Gould (1978), Tarter (1971, errata corrected 1973), and Aldrovandi and Pequignot (1973, errata corrected 1976).

A useful semiempirical formula for dielectronic recombination coefficients has been given by Burgess (1965); Aldrovandi and Pecquignot (1973) have listed approximate parameters for crudely estimating many dielectronic recombination rates at low densities. However, this process is partially suppressed if either the electron density or the infrared radiation density is high; suppression factors for several ions of interest were estimated by Davidson (1975).

Very few cross sections for charge exchange (charge transfer) have been calculated; Dalgarno and Butler (1978) and Dalgarno (1978) have reviewed this situation. Charge-exchange processes of relevance to quasars' spectra have been calculated by Blint et al. (1976), Christensen et al. (1977), and Butler et al. (1977); other possibly important reactions have been suggested by Steigman (1975). See also Chamberlain (1956), Field and Steigman (1971), Brown (1972), and Tarter et al. (1979).

Regarding excitation of $\mathrm{H}^{0}$ by electron collisions, Krolik and McKee (1978) and Crandall et al. (1974) have compiled some likely rate coefficients; see also Geltman and Burke (1970), Felden and Felden (1972), Kunc and Zgorzelski (1975), and references cited by Krolik and McKee. Collisional excitation of $\mathrm{He}^{\circ}$ has been treated by Flannery and McCann (1978).

An extremely useful table of collision strengths for several important permitted and semipermitted transitions, involving carbon and heavier ions, has been assembled by Osterbrock and Wallace (1977). Calculations of collision strengths for permitted transitions of such ions are described by Bely et al. (1963), Bely (1966), Burke and Moores (1968), Blaha (1969, 1972), Roberts (1970), Mewe (1972), Davis (1974), and Flower and Nussbaumer (1975). (In some cases, beware of a factor of 2 in the definition of $\Omega_{i j}$.) Among semiforbidden transitions, the most notable in quasars is C III] $\lambda 1909$, whose collision strength or emissivity has been discussed by Osterbrock (1970a, b), Nussbaumer (1972), Flower and Launay (1972,1973), Loulergue and Nussbaumer (1976), and Berrington et al. (1977). Other semiforbidden collision strengths are mentioned by
Nussbaumer (1972), Jackson (1973), Flower and Nussbaumer (1975), Malinovsky (1975), and Berrington et al. (1977). Forbidden lines are of less interest in quasars; their collision strengths or emissivities have been calculated or listed by Czyzak et al. (1968), Czyzak et al. (1970), Saraph and Seaton (1970), Seaton (1975), Pradhan $(1974,1976,1978)$, and Garstang, Robb, and Rountree (1978). (In some of these papers the symbol $\Upsilon$ has been used for the velocity-averaged value of $\Omega$.)

A large compilation of radiative transition rates $A_{j i}$ for permitted and forbidden (but not semiforbidden) lines is available in Wiese, Smith, and Glennon (1966); energy levels and ionization thresholds are also conveniently listed there. More recent references for permitted $A_{j i}$ 's include Heroux $(1967,1969)$, Nussbaumer (1972), Flower and Nussbaumer (1975), Morton and Smith (1973), Zeippen et al. (1977), Phillips (1979), and Czyzak and Krueger (1979). A few semiforbidden $A_{j i}$ 's have been estimated or quoted by Nussbaumer (1972), Nussbaumer and Storey (1978), Loulergue and Nussbaumer (1976), Flower and Nussbaumer (1975), and Malinovsky (1975); see also Baldwin and Netzer (1978) for a short compilation. Forbidden $A_{j i}$ 's have been lited by Garstang (1968), Nussbaumer (1971), and Nussbaumer and Rusca (1979).

Collisional ionization by free electrons is difficult to calculate; see Bely and Van Regemorter (1970), McWhirter (1975), and references therein. Collisional ionization of hydrogen is discussed by Geltman and Rudge (1963), Rudge (1963), Rau (1971), and Krolik and McKee (1978), and references therein. For other ions, see Canto and Daltabuit (1974), Burgess et al. (1977), Franco and Daltabuit (1978), Khare and Padalia (1970), Moores and Nussbaumer (1970), and references therein. The inverse process, three-body recombination, was mentioned by Bates et al. $(1962 \mathrm{a}, \mathrm{b})$ and by Bates and Dalgarno (1962).

The two-photon decay process in hydrogenic ions, and certain related collisional transitions, were discussed by Breit and Teller (1940), Spitzer and Greenstein (1951), Purcell (1952), Seaton (1955), Bethe and Salpeter (1957), and Shapiro and Breit (1959). For an indirectly related situation involving $\mathrm{He}^{\mathrm{o}}$, see Drake et al. (1969), Burke et al. (1969), Griem (1969), and Drake (1971).

Krolik and McKee (1978) have discussed various radiative processes involving the low-energy (infrared, millimeter-wave) continuum and its effects on some transitions. Raymond and Smith (1977) have listed parameters for many $x$-ray lines.

\section{CONVENTIONAL, DUSTLESS PHOTOIONIZATION MODELS FOR QUASARS' SPECTRA}

\section{A. Historical and preliminary}

Most quasars have the same emission lines that we would expect to see in a photoionized nebula; therefore it has seemed natural, since the mid-1960s, to think that quasars' line-emitting regions are photoionized. Although this surmise cannot be verified rigorously, we shall later mention several pieces of observational evidence that photoionization is more important than other ionizing and heating mechanisms in the lineemitting gas. 
In one of the first papers on the subject, Greenstein and Schmidt (1964) compared spectra of the low-redshift quasars $3 \mathrm{C} 48$ and $3 \mathrm{C} 273$ with spectra of ordinary nebulae within our Galaxy. Noting that forbidden lines in $3 \mathrm{C} 273$ are relatively weak, and therefore (presumably) collisionally deexcited, Greenstein and Schmidt proposed an electron density of the order of $3 \times 10^{6} \mathrm{~cm}^{-3}$ for this object. Higher densities have since become more fashionable, for the following reason. Forbidden lines in quasars' and some active galaxies' spectra are narrower than the permitted and semipermitted lines (see, e.g., Baldwin, 1975; Osterbrock, 1977); after some controversy (see Sec. VIII below), the linewidths are now thought to represent velocity dispersions of the emitting regions; therefore the observed forbidden lines probably arise in distinct, less-dense regions that have smaller velocity dispersions than the gas where the broad lines originate. In other words, since we can't even detect broad forbidden lines, the broadline gas must be quite dense: $n_{e} \gg 10^{7} \mathrm{~cm}^{-3}$. (In Sec. VII we shall mention one or two conceivable though unlikely ways to avoid this conclusion.) The broad-line regions are of more interest here because they are more intimately associated with the quasars' centers of activity.

Before the discovery of strongly red-shifted quasars, the Earth's atmosphere had prevented astronomers from observing ultraviolet nebular lines. By the mid1960s, several quasars' spectra had become available, and Osterbrock and Parker (1966) made some calculations to assess the idea that the observed emission lines were produced under fairly straightforward, though dense, nebular conditions. They found that quasarlike relative line intensities would result from conventional ionic abundances, rather like those in some galactic nebulae, at temperatures around $15000 \mathrm{~K}$. This did not constitute a derivation of chemical composition, because alternative sets of elemental abundances and ionization ratios would also have produced satisfactory spectra, at slightly different temperatures. Rather, the significance of Osterbrock and Parker's paper was that the order of magnitude of the proposed temperature lent plausibility to the idea of photoionization. If $T$ $z 10^{5} \mathrm{~K}$ had been found to give the best results, then other heating mechanisms would have been indicated; but only photoionization produces doubly and triply ionized species at equilibrium temperatures below $30000 \mathrm{~K}$.

Photoionization posed a geometrical question. Given the electron density, one can estimate the volume of emitting gas required to produce the observed emission-line luminosities. If, as supposed in many early papers, this gas were concentrated in a single cloud around a compact continuum source, then for reasonable densities the cloud would be considerably less than a light-year across and would be optically thick to electron scattering. The radiation/gas density ratio $U_{\mathrm{H}}$ would be much higher than the values required in the photoionization models discussed below. However, as Schmidt (1964) remarked, line-emitting gas may be distributed less simply. By analogy with galactic nebulae and supernova remnants, which are very inhomogeneous, we may expect the gas to be in the form of numerous cloudlets, filaments, or sheets, with a small overall "filling factor" (fraction of volume actually occupied). A thin spherical shell can serve as an idealized approximation for some purposes. Shklovsky (1965) pointed this out, and observations by Sandage, Westphal, and Strittmatter (1966), of variations of continuum flux concurrent with nonvariations in line intensities in the quasar $3 \mathrm{C} 446$, also suggested that the line-emitting gas is not distributed compactly. [Shields, Oke, and Sargent (1972) later gave the same argument for the active galactic nucleus 3C 120.] Shklovsky (1965) and Burbidge, Burbidge, Hoyle, and Lynds (1966) qualitatively discussed the stratification of photoionized regions in quasars-although the latter authors supposed that the linewidths were due to electron scattering rather than velocity dispersions.

A few more arguments were adduced in favor of photoionization in the nuclei of Seyfert galaxies as well as quasars. Searle and Sargent (1968) noticed that the ratio of the $H \beta$ recombination line intensity to the underlying continuum brightness is roughly constant in the spectra of many Seyferts and quasars, over a wide range of absolute luminosities. Because a recombination line from a photoionized gas indirectly measures the number of absorbed ionizing continuum photons, this is suggestive of photoionization, and the numerical values are consistent with a moderate power-law continuum spectrum. Oke, Neugebauer, and Becklin (1970) similarly noted that the extrapolated power-law continua of quasars seem to contain enough ionizing flux to account for the emission lines. Souffrin $(1969 a, b)$ remarked that the absence of certain visual-wavelength emission lines, arising from highly excited levels, is evidence against the high temperatures that would probably result from strong nonphotoionization heating mechanisms. It should also be mentioned that a few early arguments against photoionization (e.g., see Osterbrock and Parker, 1965; Oke and Sargent, 1968) did not take the properties of power-law and soft-x-ray continua fully into account.

The earliest period of quasar-spectrum analysis ended when Bahcall and Kozlovsky (1969a, b) produced models that can be regarded as logical and major extensions of the Osterbrock and Parker (1966) approach. Like Osterbrock and Parker, Bahcall and Kozlovsky specified particular temperatures; but instead of guessing the ionization ratios for each element, they calculated position-dependent photoionization equilibria in modified spherical configurations with particular gas densities and with power-law ionizing spectra emanating from a central source. Chan and Burbidge (1975) described later calculations of the same type.

The next, very important step was to calculate equilibrium temperatures as well as ionization ratios. In classical nebular analysis, applicable at low electron densities where collisional deexcitation is not too important, temperatures can sometimes be deduced from observed intensity ratios of forbidden lines involving different excited levels of the same ion species. Using such temperatures, one can calculate relative emissivities for other observed lines, and thus find the relative ionic abundances required to produce the lines. But no direct temperature indicators are available in quasars' 
spectra (aside from one or two crude limits). Instead, one can only hope that requiring self-consistency of ionization and thermal structures-modeling the flow of energy through the system-will provide additional information by eliminating almost all of "parameter space," where the parameters include elemental abundances. We shall describe how this might work in Sec. III.C; meanwhile, we emphasize that the thermal equilibrium is just as important as the ionization equilibrium! (It is relevant to note that in certain parts of the models by Bahcall and Kozlovsky, the assumed temperatures made collisional ionization locally more important than photoionization; in effect, some arbitrary extra heating was introduced into the system when thermal equilibria were not calculated.) In the following, the term "photoionization calculation" will be meant to include thermal as well as ionization equilibria as functions of position.

Photoionization calculations, intended to apply to astronomical $\mathrm{x}$-ray sources, were described by Williams (1967), Tarter, Tucker, and Salpeter (1969), and Tarter and Salpeter $(1969)^{5}$; except for deficiencies in some ionic parameters, these were essentially suitable for application to quasars. They were in fact applied to Seyfert galaxies (Williams and Weyman, 1968; Tucker and Tarter, 1968), mainly to reproduce the observed visual-wavelength lines (ultraviolet lines in Seyferts' spectra could not be observed at that time). However, one can argue that the most appropriate use for photoionization calculations is to analyze the ultraviolet permitted and semiforbidden lines, rather than the visual-wavelength forbidden lines, because (1) in many objects the ultraviolet lines are more representative of the denser, inner regions, (2) the ultraviolet lines account for most of the cooling at densities above $10^{6.5}$ $\mathrm{cm}^{-3}$, and therefore are essential in a photoionization calculation, and (3) the forbidden lines are too density dependent to be reliably modeled for Seyfert nuclei and quasars. Until adequate ultraviolet spectra of low-redshift objects have become available, high-red-shift quasars are the most natural subjects for photoionization analyses.

Tarter and Salpeter (1969) made some comments regarding quasars, but the first detailed application of self-consistent photoionization calculations to quasars was described by Davidson (1972). In order to include both ultraviolet and visual-wavelength lines (which were not all observable for any one object), a "composite" spectrum was used for comparison. The conclusions were as follows:

(1) To the extent that they were known at the time, quasars' spectra could be reproduced by photoionized models. The only line which seemed difficult to explain was [O II] $\lambda 3727$, which is collisionally deexcited at rather low densities. But this line was very faint in the observed spectra and could probably be due to gas at distances of hundreds or thousands of light-years from each quasar-perhaps interstellar material in galaxies

${ }^{5}$ Hjellming (1966), Goodson (1967), Harrington (1968), Rubin (1968), and Flower (1969) applied similar calculations to ordinary galactic nebulae illuminated by hot stars. containing the quasars. (Today, [O II $] \lambda 3727$ is known to be a narrow line.)

(2) Even without data on linewidths, there was reason to suspect that the ultraviolet lines originated in denser gas than the forbidden lines. Densities of $10^{8}$ $\mathrm{cm}^{-3}$ or more were most suitable for the "dense component."

(3) The most straightforward models involved ionization parameters $U_{\mathrm{H}}$ just above $10^{8} \mathrm{~cm} \mathrm{~s}^{-1}$ in the denser gas. Such values entailed ionizing radiation pressures which were smaller than the gas pressures (though not enormously smaller); this must have dynamical implications.

(4) With relative elemental abundances resembling those in our own Galaxy, successful models were easy to construct. But if heavy elements were assumed to be very "overabundant" or "underabundant" relative to hydrogen, then some rather intricate difficulties were encountered. However, no proof was given that unconventional abundances could not be made to work.

Shortly thereafter, MacAlpine (1972) produced photoionization models at about the same level of calculative sophistication. He explored the consequences of varying several parameters, and emphasized that heavy ions can play a non-negligible role in absorbing $x$-ray energy. [Some of the early calculations appear to have neglected inner-electron-shell photoionization. This is not true of the Davidson (1972) or of course the MacAlpine (1972) discussions.] In many of MacAlpine's models, the assumed helium/hydrogen abundance ratio was very low, as suggested by Bahcall and Kozlovsky (1969a) and Osterbrock and Parker (1966) on the basis of the observed weakness of the He II $\lambda 4686$ and $\lambda 1640$ lines, especially in the quasar $3 \mathrm{C} 273$. However, as noted by Williams (1971), these are recombination lines, whose weakness may indicate a shortage of $\mathrm{He}^{+}$-ionizing photons $(\varepsilon>54.4 \mathrm{eV})$ rather than a shortage of helium. Subsequent observations of various quasars (e.g., see Baldwin, 1975) have revealed HeI lines whose intensities are not easy to analyze (see Sec. IV.D below) but which appear consistent with a "normal" helium abundance, $n_{\mathrm{He}} / n_{\mathrm{H}} \approx 0.1$.

Later photoionization calculations have been applied to quasars by Scargle, Caroff, and Tarter (1974), Davidson (1973, 1977b), Shields (1976), Netzer (1976, 1977, 1978), Baldwin and Netzer (1978), Daltabuit, MacAlpine, and Cox (1978), Ferland and Netzer (1979), and Shuder and MacAlpine (1979); some of their implications will be discussed below. Applications to Seyfert and similar galactic nuclei have been made by Yankulova (1974), Shields (1974), Shields and Oke (1975), and others; discussions by Bergeron and Souffrin (1971, 1974) are also relevant.

In Sec. III.B we shall give a specific example of a photoionization model for a particular quasar, and in Sec. III.C we describe the effects of varying the assumed parameters. But first, a few general remarks are necessary.

To begin with, there are some practically unanswerable questions about the geometrical arrangement of gas associated with a quasar. As mentioned above, plausible homogeneous-density models are difficult to construct. We suppose instead that a quasar has num- 
erous clouds, filaments, or sheets of gas, perhaps immersed in a transparent lower-density medium; a thin shell is conceivable either as an approximate representation or as an alternative configuration, while disklike configurations have also been suggested, e.g., by Shields (1977). We shall see that the whole assembly, excepting the forbidden-line emission regions, is likely to be 1 to 1000 light-years across, which, at large cosmological distances, will probably not be resolvable at visual wavelengths in the near future. There are reasons (to be mentioned later) for thinking that each individual cloud is optically thick to most ionizing photons $(13.6 \mathrm{eV}<\varepsilon \lesssim 1 \mathrm{keV})$, so ionizing radiative transfer must be considered. In many calculations, the surface of an individual cloud, filament, or sheet is approximated by a plane-parallel geometry. Proper treatment of convex surfaces would be much more difficult, and in almost any case we would not know how to select the right shape and size. Fortunately, because the secondary ionizing radiation is less important than the incident radiation, geometrical details do not seem crucial to the results; in fact, modified "on-the-spot" approximations (see Sec. II.A) are sometimes good enough to be useful. For these reasons, in this review we do not discuss the details of the iterative diffusionlike radiative transfer treatments, which are not particularly sophisticated in most calculations anyway. "Shadowing", and the effects of clouds' secondary radiation upon each other, have not received much attention; nor have possible internal pressure and density nonuniformities in the clouds.

A quasar's photoionized gas must have some distribution of densities and values of the ionization parameter $U_{\mathrm{H}}$. In practice, the best representation that we can use in a model is to include one, two, or three distinct "components" having particular values of $n_{e}$ and $U_{\mathrm{H}}$; there are not enough observational data to support anything more complex. Some published models have continuous distributions of $U_{\mathrm{H}}$ (although the authors usually do not specify them in these terms), but the particular distributions chosen are rather arbitrary. (Example: one can suppose that gaseous clouds, all of one size and internal density, are spread uniformly throughout a spherical volume centered on a small ionizing source. This gives a particular distribution of $U_{\mathrm{H}}$ values.)

There are some probable limits on gas densities and temperatures in quasars' broad-line emission regions. The most nearly straightforward lower limits on density are provided by the absence of broad [O III] $\lambda 4363$ and $\lambda 5007$ emission (see Baldwin, 1975), which would originate in the same ionized zones as the prominent $C$ IV $\lambda 1549$ line. If the carbon/oxygen abundance ratio is not far from "normal," and if the forbidden lines' absence is due to collisional deexcitation, then electron densities in the broad-line gas must exceed $10^{7} \mathrm{~cm}^{-3}$, probably by a considerable factor. Upper limits on densities are suggested by the presence of semiforbidden lines, especially C III] $\lambda 1909$, whose importance in this regard was noted by Osterbrock (1970). The observed intensity of $\lambda 1909$, relative to C IV $\lambda 1549$, is large enough to require some care to reproduce in photoionization models, even if collisional deexcitation is unimportant. The critical deexcitation density for $\lambda 1909$ is about $10^{9.6} \mathrm{~cm}^{-3}$ (note that many pre-1977 references quote an erroneously higher value). Therefore we probably require average electron densities of less than about $10^{10} \mathrm{~cm}^{-3}$, although specially adjusted models with unusually high carbon abundances and low $U_{\mathrm{H}}$ values can be made to work at densities up to $10^{10.2}$ or $10^{10.4} \mathrm{~cm}^{-3}$. Considerations outlined in Secs. IV.C and IV.D below make us suspect that the actual densities are somewhat above $10^{9} \mathrm{~cm}^{-3}$.

The C III] $\lambda 1909$ line also provides a useful temperature limit, because it is observed to be brighter than the permitted C III $\lambda 977$ line (Bahcall and Oke, 1971; Baldwin and Netzer, 1978). At relevant densities, these two lines should have an energy-emissivity ratio

$$
\frac{I(\lambda 977)}{I(\lambda 1909)} \approx 10\left(1+\frac{n_{e}}{n_{c}}\right) e^{-T_{c} / T},
$$

where $T_{c}=71900 \mathrm{~K}$ and $n_{c} \approx 10^{9.6} \mathrm{~cm}^{-3}$. Although $\lambda 977$ is in an observationally unfavorable part of the spectrum-shortward of $L \alpha$, where many absorption lines occur in high-red-shift spectra-it is thought to be typically less than half as bright as $\lambda 1909$; therefore the appropriate average $\mathrm{C}^{2+}$ temperature cannot exceed $25000 \mathrm{~K}$. This is fairly good evidence in favor of photoionization and against strong additional heating mechanisms.

(It is true that the $\mathrm{C}$ IV $\lambda 1549$ and $\mathrm{C}$ III $\lambda 977$ lines, mentioned in the preceding arguments, are potentially subject to resonance-scattering difficulties as discussed in Sec. IV below. Except for possible absorption by dust grains, however, these difficulties are estimated to be harmless for $n_{e} \lesssim 10^{10} \mathrm{~cm}^{-3}$. One might also try to reduce the implications of $\mathrm{CIII}] \lambda 1909$ by supposing that it is produced by a different component of gas than C IV $\lambda 1549$ and other lines; but then upon calculation it turns out that the $\mathrm{CIII}$ ] component must be comparable in overall importance to the $\mathrm{C} \mathrm{IV}$ component, so the estimated limits remain significant.)

Finally, we note that the models discussed in Secs. III. B and III.C are mainly concerned with ultraviolet lines, whose photons are assumed to escape from the ionized regions, without much absorption by dust grains, for example. During the past two or three years, very serious discrepancies between calculated and observed spectra have appeared, when the entive rest-wavelength range of 1000-7000 $\AA$ is included. This problem will be discussed separately in Sec. $V$ below; it appears that wavelength-dependent extinction by dust grains is indeed important. Therefore the immediately following discussion represents only a first approximation to a truly complex situation.

\section{B. A specific photoionization calculation}

We shall now describe a concrete example of a photoionization model; more general discussions will then be given by Secs. III. C and V. Our example is a model described by Baldwin and Netzer (1978), with the unimportant addition of some effects involving scattering and slight collisional deexcitation of permitted lines. It was intended to produce ultraviolet line intensities which resemble the observed spectrum of PHL 938, a fairly typical quasar. PHL 938 has a red shift $z=1.95$, so that its ultraviolet spectrum (1000-3000 $)$ appears to us at 
ground-based observable wavelengths 3000-9000 A.

The assumed geometry of the gas is semi-infinite, with an ionizing continuum perpendicularly incident upon the plane boundary. This is the most convenient approximation to an illuminated surface of a representative cloud of filament or sheet, which is optically thick to the ionizing photons that are produced elsewhere in the quasar. A finite spread in these photons' angles of incidence would not be terribly important, if $U_{\mathrm{H}}$ is considered in terms of radiation density rather than radiation flux. The incident ionizing continuum, which can be joined smoothly to the observed slope of the continuum of PHL 938, has the form $F(\varepsilon) \sim \varepsilon^{-s} e^{-\varepsilon / \varepsilon_{0}}$, with $s=0.8, \varepsilon_{0}=0.7 \mathrm{keV}$ (the chosen value of $\varepsilon_{0}$ is rather arbitrary). At the illuminated surface, $U_{\mathrm{H}}=10^{8.04} \mathrm{~cm} \mathrm{~s}^{-1}$ and $n_{e}=10^{9.4} \mathrm{~cm}^{-3}$. The gas pressure is constrained to be uniform. The relative elemental abundances are $n_{Z} / n_{\mathrm{H}}=(100,1.4,1.1,2.6,0.4,0.12,0.12,0.06$, and $0.1) \times 10^{-3}$ for $Z=\mathrm{He}, \mathrm{C}, \mathrm{N}, \mathrm{O}, \mathrm{Ne}, \mathrm{Mg}, \mathrm{Si}, \mathrm{S}$, and $\mathrm{Fe}$. (Except for helium, these values are unusually large by astrophysical standards; but there is no need to discuss this point yet. See the next section.)

With these input parameters, some details of the calculated photoionization equilibrium, as functions of position $x$ in the gas, are shown in Figs. 5 and 6 . Throughout most of the ionized zones, $T$ is between 13000 and $16000 \mathrm{~K}$. The ionized thickness is $x_{\mathrm{H}} \approx 10^{11.4}$ $\mathrm{cm}$, which is somewhat larger than that predicted by Eq. (2.5), partly because of secondary ionizing photons. Note in Fig. 6 that the optical depth $\tau_{\mathrm{H}}$ for photon energies just above the hydrogen threshold $I_{\mathrm{H}}=13.6 \mathrm{eV}$ is of the order of 50 at the ionization front where $n\left(\mathrm{H}^{0}\right)=n\left(\mathrm{H}^{+}\right)$. Immediately beyond the ionization front, the temperature remains above $10000 \mathrm{~K}$, because some ionization is caused there by penetrating soft-x-ray photons $(\varepsilon>200 \mathrm{eV})$. Helium atoms absorb many of these photons; although the rates are too small to produce a large equilibrium $\mathrm{He}^{+} / \mathrm{He}^{0}$ ratio, the resultant heating is very effective because each freed electron is given

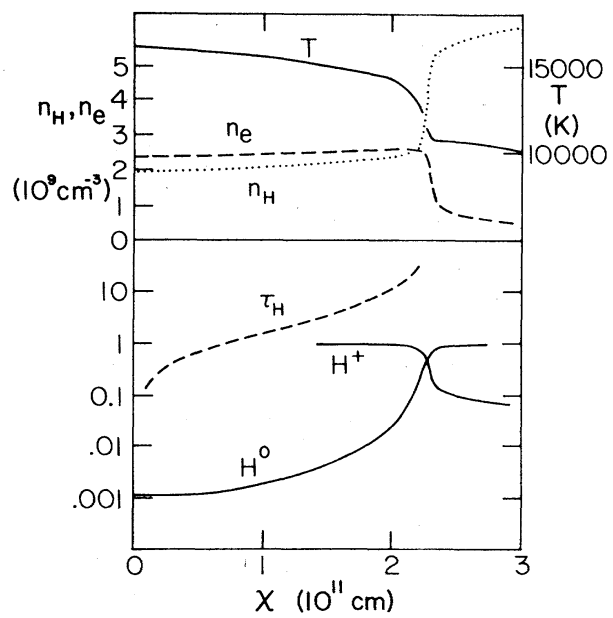

FIG. 5. Various quantities as functions of position, in a particular photoionization model with uniform gas pressure. $T$ is kinetic temperature, $n_{e}$ is electron density, and $n_{\mathrm{H}}$ is hydrogen density (including ions as well as atoms). In the lower part of the figure, the threshold hydrogen photoionization optical depth $\tau_{\mathrm{H}}$ is shown along with the equilibrium fractions of $\mathrm{H}^{+}$and $\mathrm{H}^{0}$.

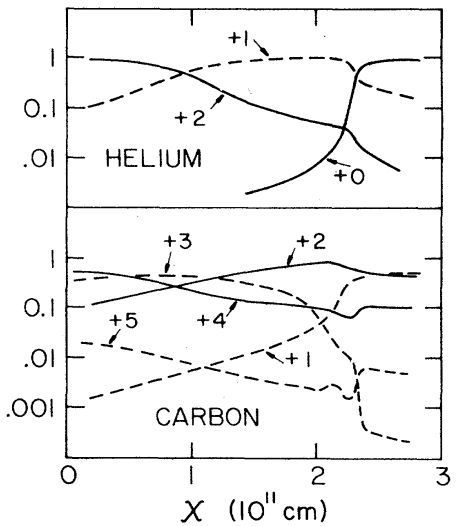

FIG. 6. Equilibrium ionization fractions for helium and carbon, as functions of position in the same photoionized region shown in Fig. 5 .

a large initial energy. [Even before they can be thermalized by elastic collisions, such energetic electrons cause excitations of atoms, especially $\mathrm{H}^{0}$. See Shull (1979).] Temperatures of several thousand kelvins persist far beyond $x=10 x_{\mathrm{H}}$.

Examination of the carbon ionization fractions (Fig. 7) reveals other nontrivial effects. Carbon is largely $\mathrm{C}^{2+}$ in the $\mathrm{H}^{0}$ zone because carbon cross sections at $\mathrm{x}$-ray energies are larger than those of hydrogen and helium. Some charge-exchange reactions are important whereever $n\left(\mathrm{H}^{0}\right) / n_{e}$ or $n\left(\mathrm{He}^{0}\right) / n_{e}$ exceeds 0.01 ; for carbon, only $\mathrm{C}^{3+}+\mathrm{H}^{0} \rightarrow \mathrm{C}^{2+}+\mathrm{H}^{+}$was included in this calculation, but higher-ionization reactions are probably important too, so the $\mathrm{C}^{4+}$ and $\mathrm{C}^{5+}$ fractions have most likely been overestimated.

Even if some aspects of the calculation are unreliable in the $\mathrm{H}^{0}$ zone, the calculated emission spectrum will not be correspondingly bad-because most of the energy absorbed in this zone goes into collisionally excited hydrogen emission, almost regardless of details. However, most of the Mg II $\lambda 2798$ emission comes from this same zone and may be affected by such details. The observed prominence of this line is evidence that at least some gas is optically thick to ionizing photons.

Calculated relative intensities of some ultraviolet lines are shown in Table I, along with observed values for PHL 938. The $L \alpha, \mathrm{CIV}, \mathrm{C}$ III], and Mg II intensities are fairly successful, and with further "tuning" a plausible model can be constructed. Note that the highexcitation NV $\lambda 1240$ line, and part of He II $\lambda 1640$, probably require a "second component" of gas, which may be optically thin and which either has a relatively high $U_{\mathrm{H}}$ or else is ionized by some additional process. See Baldwin and Netzer (1978) for details.

Some of the geometrical parameters are interesting. First, it turns out that the observed line intensities, measured relative to the underlying continuum brightness of PHL 938, can be explained by supposing that the gaseous regions (clouds, filaments, or whatever) intercept only a few percent of the quasar's ionizing photons.

Second, with the assumed values of $U_{\mathrm{H}}$ and $n_{e}$, the energy density of ionizing radiation in the gas is about $1 / 15$ as large as the thermal kinetic energy density in 
TABLE I. Relative line intensities (energy fluxes) from a photoionization calculation, compared with the spectrum of the quasar PHL 938.

\begin{tabular}{lccc}
\hline \multicolumn{2}{c}{ Line } & Calculation & PHL 938 \\
\hline C III & $\lambda 977$ & 2.7 & $\ldots$ \\
O VI & $\lambda 1034$ & 1.1 & $\ldots$ \\
L $\alpha$ (H I $\lambda 1215)$ & $100^{\text {a }}$ (by def.) & 100 (by def.) \\
N v & $\lambda 1240$ & 5.8 & $36 \pm 16$ \\
Si IV & $\lambda 1397$ & 1.2 & $\lesssim 30 ? ?$ \\
O IV] & $\lambda 1402$ & 2.1 & $4 \pm 1.6$ \\
N IV] & $\lambda 1486$ & 4.0 & $45 \pm 10$ \\
C IV & $\lambda 1549$ & 45 & $6 \pm 2$ \\
He II & $\lambda 1640$ & 3.0 & $3 \pm 1.5$ \\
O III] & $\lambda 1663$ & 3.6 & $1 \pm 0.5 ?$ \\
N III] & $\lambda 1750$ & 4.4 & $19 \pm 4$ \\
C III] & $\lambda 1909$ & 22 & $18 \pm 6$ \\
Mg II & $\lambda 2798$ & 28 & 4 \\
\hline \hline
\end{tabular}

${ }^{\text {a }} 34 \%$ of $L \alpha$ is due to recombination; $66 \%$ is due to collisional excitation of $\mathrm{H}^{0}$.

the gas and amounts to roughly $10^{-3} \mathrm{erg}^{-3}$. (This is about 20 times the energy density of sunlight on the Earth.) PHL 938 produces an ionizing luminosity of perhaps $10^{47 \cdot 3} \mathrm{erg} \mathrm{s}^{-1}$ (about $10^{13.7}$ times the total luminosity of the sun), so the characteristic distance of the photoionized regions from the central object-if there is one-would be of the order of $10^{19.3} \mathrm{~cm}$ or $20-30$ lightyears.

But the characteristic thickness of each photoionized region is only $x_{\mathrm{H}} \approx 10^{11.3} \mathrm{~cm}$ ! Either the gas clouds (or filaments, or whatever) are exceedingly small compared with the overall configuration, or only a tiny fraction of each cloud is ionized, or both. Moreover, the characteristic dynamical and ionization time scales in the ionized gas, $x_{\mathrm{H}} / w$ and $x_{\mathrm{H}} / U_{\mathrm{H}}$ (where $w=$ isothermal speed of sound $\approx 15 \mathrm{~km} \mathrm{~s}^{-1}$ ), are around 2 days and $\frac{1}{2} \mathrm{~h}$, respectively. Evidently, the photoionized gas, overall, is in some sort of quasisteady state. Changing the parameters $U_{\mathrm{H}}$ and $n_{e}$, within plausible limits, does not qualitatively alter this remarkable situation.

Incidentally, if we speculate that the photoionized regions have the same pressure as some thinner medium (hotter gas, electromagnetic fields, or high-energy particles) which fills the spaces between, then the energy density of this medium is just above $10^{-2} \mathrm{erg} \mathrm{cm}^{-3}$, with the parameters used in the model. The energy contained in the region 50 light-years across would be of the order of $10^{57} \mathrm{erg}$, equivalent to less than 100 years of luminous output by the quasar.

\section{Varying the assumed parameters}

A model like the one sketched above, by itself, is only speculatively useful, because other models may give similar spectra. We must consider the effects of the various input parameters, to see how much the model could have been changed without seriously affecting the emitted spectrum. We should also be prepared to deal with other spectra, which may differ from that of PHL 938. Investigations in this manner have been sketched by Davidson $(1973,1975,1977 b)$ and Shields (1976). Throughout the following discussion, remember that most atomic and ionic parameters, upon which we depend, are imperfectly known. Many have uncertainties of $10 \%$ or $20 \%$; some, especially those involving faint emission lines, are worse.

To begin, suppose that the photoionizing continuum is adequately parameterized by $F(\varepsilon) \sim \varepsilon^{-s} e^{-\varepsilon / \varepsilon_{0}}$ in the relevant range $13.6 \mathrm{eV} \lessgtr \varepsilon \lessgtr 1 \mathrm{keV}$. Unfortunately, we can only guess the values of $s$ and $\varepsilon_{0}$, by extrapolating the observed $1 \mathrm{eV} \lessgtr \varepsilon \lessgtr 15 \mathrm{eV}$ continua and by watching for inconsistencies in the calculated emission spectra, and, perhaps, in x-ray observations. Suppose that electron densities are in the previously suggested range, $10^{7} \mathrm{~cm}^{-3}<n_{e} \lessgtr 10^{10} \mathrm{~cm}^{-3}$. Suppose also that the helium/hydrogen abundance ratio is about "normal," $n_{\mathrm{He}} / n_{\mathrm{H}} \approx 0.1$. In many ordinary galactic nebulae, $n_{\mathrm{He}} / n_{\mathrm{H}}$ can be measured by comparing visual-wavelength recombination lines of both HeI and He II with those of hydrogen; this works well because $\mathrm{He}^{+}$and $\mathrm{He}^{++}$together fill nearly the same regions as $\mathrm{H}^{+}$in those nebulae, and because recombination lines' ratios are insensitive to temperatures and densities there. Quasars' recombination lines are trickier (see Sec. IV.D regarding the He I lines, which are most important), but the "derived" helium/hydrogen ratio still seems to be close to that quoted above-presumably because most of the helium was synthesized in the primordial universe before stars or quasars existed. The remaining parameters are a characteristic value (or values) of $U_{\mathrm{H}}$ and the abundances of heavy elements, chiefly carbon, nitrogen, and oxygen. Carbon is especially important, because C IV $\lambda 1549$ is a major coolant while the ratio of the C III ] $\lambda 1909$ and C IV $\lambda 1549$ lines is an indicator of $U_{\mathrm{H}}$. As is usually the case in nebular astrophysics, we must assume that the relative abundances are fairly uniform throughout the line-emitting regions of a quasar, for otherwise the situation becomes hopelessly complicated. Regarding the internal structures of the ionized cloudsconstant pressure, or constant density, or whateversee the references cited.

Figure 7 schematically shows $U_{\mathrm{H}}$ dependences of

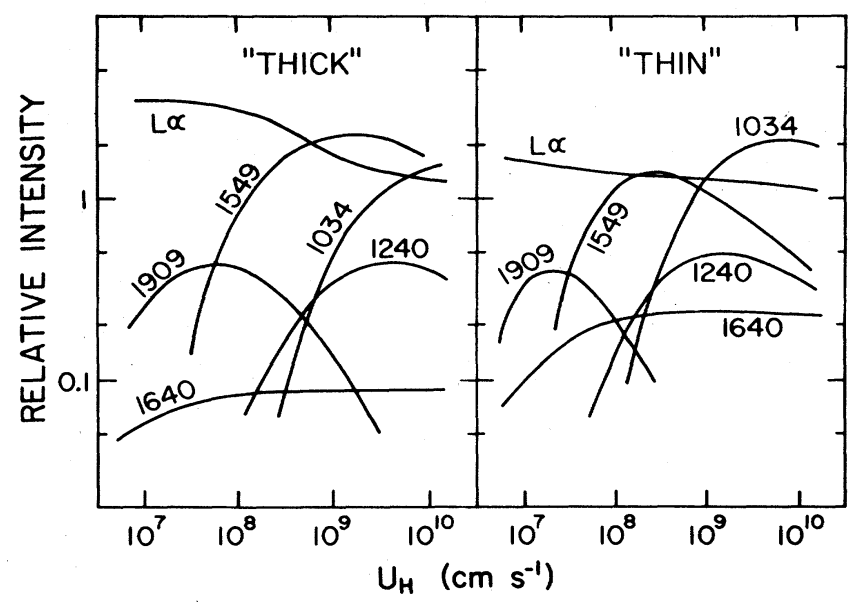

FIG. 7. Relative line intensities produced in photoionized regions with a particular ionizing-continuum shape and particular elemental abundances, as functions of the ionization parameter $U_{\mathrm{H}}$ " "Thick" models have $\tau_{\mathrm{H}}>100$; "thin" models have $\tau_{H}<1$. In this figure, the intensities have been normalized so that the recombination part of $L \alpha$ is equal to unity. 
most of the brightest emission-line intensities (remember, we are always referring to the broad-line rather than the forbidden-line regions): OVI $\lambda 1034, L \alpha \lambda 1215$, NV $\lambda 1240$, C IV $\lambda$ 1549, He II $\lambda 1640$, and C III ] $\lambda 1909$. These account for most of the cooling. The figure, which is slightly modified ${ }^{6}$ from those used by Davidson (1977b), applies to the case of a power-law spectrum $\left(s=1, \varepsilon_{0} \rightarrow \infty\right)$ with rather conventional abundances $n_{Z} / n_{\mathrm{H}}=0.1,0.0004,0.0002,0.0008$ for $Z=\mathrm{He}, \mathrm{C}, \mathrm{N}, \mathrm{O}$. These CNO abundances are smaller than those in the model described in Sec. III.B. Figure 7 is nearly valid for $10^{6.5} \mathrm{~cm}^{-3} \lessgtr n_{e} \lessgtr 10^{9} \mathrm{~cm}^{-3}$; above $10^{9} \mathrm{~cm}^{-3}$, the C III ] $\lambda 1909$ intensity gradually diminishes in proportion to $n_{c} /\left(n_{c}+n_{e}\right)$ with $n_{c} \approx 10^{9.6} \mathrm{~cm}^{-3}$, while the other intensities are valid up to about $10^{10} \mathrm{~cm}^{-3}$. The $\mathrm{Mg}$ II $\lambda 2798$ and Balmer lines have not been included, but may be considered, in effect, to be a part of the calculated $L \alpha$ intensity. The left half of the diagram shows the spectra of gas clouds which are optically thick to ionizing photons, while the right half refers to optically thin, homogeneous clouds. Equilibrium temperatures, among different ionization zones and values of $U_{\mathrm{H}}$, vary from 10000 to $50000 \mathrm{~K}$ in the models represented by the figure.

C III ] $\lambda 1909$ is generally observed to be less intense than C IV $\lambda 1549$ in quasars, typically by a factor of 2 or 3. In Fig. 7, this occurs at $U_{\mathrm{H}}$ slightly above $10^{8} \mathrm{~cm} \mathrm{~s}^{-1}$ for a thick cloud, or slightly below $10^{8} \mathrm{~cm} \mathrm{~s}^{-1}$ for a thin one. Many calculations have shown that this result is insensitive to the carbon abundance, being essentially a measure of the $\mathrm{C}^{3+} / \mathrm{C}^{2+}$ ionization ratio; and regarding different ionizing spectra, we note that the $\mathrm{C}^{3+} / \mathrm{C}^{2+} \mathrm{ra}-$ tio depends upon photons just above the $\mathrm{C}^{2+}$ threshold of $48 \mathrm{eV}$. If $n_{e}>10^{9} \mathrm{~cm}^{-3}$ so that C III ] $\lambda 1909$ is collisionally deexcited, then $U_{\mathrm{H}}$ must be decreased (but not very much). The suggested order of magnitude of $U_{\mathrm{H}}$ is consistent with the observed brightness of $\mathrm{Mg}$ II $\lambda 2798$, which depends upon $U_{\mathrm{H}}$ as well as other parameters.

At the indicated value of $U_{\mathrm{H}}$ on the left side of Fig. 7, $L \alpha$ is 2 or 3 times as bright as C IV $\lambda$ 1549. Since this matches the observed quasarian ratios, we deduce that the assumed carbon/hydrogen abundance ratio may be about right (even though it differs from the model in Sec. III.B; we'll explain this discrepancy later). If we were using the thin version, where the CIV line in Fig. 7 is unacceptably bright relative to $L \alpha$, we would readjust the carbon abundance downward. Some crude proportionality relations given by Davidson (1973) are useful for estimating the dependences of $\mathrm{CIII}] / \mathrm{CIV} / L \alpha$ intensities upon $n_{\mathrm{C}} / n_{\mathrm{H}}$.

We must now digress on a matter of nebular principle, concerning the above "derivation" of $n_{\mathrm{C}} / n_{\mathrm{H}}$. The C IV $/ L \alpha$ intensity ratio depends significantly upon $n_{\mathrm{C}} / n_{\mathrm{H}}$ only because part of the $L \alpha$ emission is due to collisional excitation of $\mathrm{H}^{0}$ atoms. If $L \alpha$ were purely a recombination line (intensity =1 in Fig。 7), then it would represent merely the number of recombination events, which for $T \ll 10^{5} \mathrm{~K}$ play only a minor role in cooling the gas (see Sec. II). The temperature would necessarily adjust it-

\footnotetext{
${ }^{6}$ Modified to take into account charge exchange, and partial collisional deexcitation of C III] $\lambda 1909$ at $n_{e}=10^{9} \mathrm{~cm}^{-3}$.
}

self so that carbon lines, plus other heavy-ion lines, provide adequate cooling. C IV $\lambda 1549$ is observably the most important of these lines, while C III ] $\lambda 1909$ is also quite prominent. The $\mathrm{CIV} / L \alpha$ intensity ratio would therefore depend almost entirely upon the ratio of required cooling rate to hydrogen recombination rate; this ratio is essentially the heating rate per photoionization event, which is a measure of the average photon energy and has little to do with the carbon abundance. But in reality, for low or moderate values of $U_{\mathrm{H}}$, enough $\mathrm{H}^{0}$ atoms are mixed in with the $\mathrm{H}^{+}$to allow collisionally excited $L \alpha$ to assume a significant and abundance-dependent share of the cooling burden, making the observed line ratios dependent upon the carbon abundance. Unfortunately, this dependence is not as strong as we would like (cf. the proportionality relations-Davidson, 1973), because of the limited extent of the zone where C IV $\lambda 1549$ production and collisional $\mathrm{H}^{0}$ excitation occur together. Much of the collisionally excited $L \alpha$ originates in the $\mathrm{H}^{+} / \mathrm{H}^{0}$ transition zone, where it dominates the cooling; much of the CIV emission originates in a more thoroughly ionized zone, where it dominates the cooling; and only an intermediate zone gives a strongly abundance-dependent contribution to the spectrum.

Moreover, the actual amount of necessary cooling depends upon the ionizing continuum. In the exemplary model described in Sec. III.B, $n_{\mathrm{C}} / n_{\mathrm{H}}$ was more than 3 times the value used in Fig. 7. Aside from minor calculative differences, this apparent discrepancy arises because (1) the ionizing spectrum with $s=0.8, \varepsilon_{0}=0.7$ $\mathrm{keV}$ has more photons around $100 \mathrm{eV}$ than the $s=1$ power law, and such photons cause effective heating; (2) C III ] $\lambda 1909$ is reduced by about $40 \%$ by collisional deexcitation at the density adopted by Baldwin and Netzer for PHL 938, and this leads to a reduced value of $U_{\mathrm{H}}$, which in turn reduces the fraction of carbon which is C IV; and (3) both the $L \alpha / \lambda 1549$ and the $\lambda 1549 / \lambda 1909$ intensity ratios are close to 2 in PHL 938, rather than 2.5 or 3 . To further emphasize the difficulties of the situation, we note that some early estimates (Davidson, 1973) made $n_{\mathrm{C}} / n_{\mathrm{H}}$ surprisingly small, partly because of calculative simplifications but mainly because the adopted ionizing continuum had a rather low cutoff parameter $\varepsilon_{0}=0.2 \mathrm{keV}$ (which may or may not be realistic), while the $L \alpha / \lambda 1549$ and $\lambda 1549 / \lambda 1909$ line ratios were then thought to be 3 or 4 rather than 2 or 3 .

Returning our attention to Fig. 7, we see that for $U_{\mathrm{H}} \approx 10^{8} \mathrm{~cm} \mathrm{~s}^{-1}$, OVI $\lambda 1034$ and $\mathrm{NV} \lambda 1240$ are much weaker than observed. If the adopted continuum is not far wrong, these high-ionization lines must originate in another component of gas, which either has higher values of $U_{\mathrm{H}}$, or else is ionized and heated by some additional mechanism. In order to make $\mathrm{NV} \lambda 1240$ about as bright as OVI $\lambda 1034$, while both are comparable to $L \alpha$ in the second component, it is probably necessary to increase the nitrogen/oxygen abundance ratio [Osmer and Smith (1976) noted this point]. The high-excitation component of gas may be optically thin, except perhaps to $\mathrm{He}^{+}$-ionizing photons just above $54.4 \mathrm{eV}$ and to radiofrequency waves (see Sec. VII.C below). As remarked by Davidson (1973), an optically thin secondary component is also useful for providing He II $\lambda 1640$ emission. Note: we do not insist that the two components are well 
defined and distinct, but rather, that "two components" merely provide the simplest representation of what may be a disagreeably complex situation, involving some continuous distribution of values of $U_{\mathrm{H}}$ and $n_{e}$.

By going into the numerical details of a two-component model (e.g., see Davidson, 1977b, or Baldwin and Netzer, 1978), one finds that the major, low- $U_{\mathrm{H}} \mathrm{com}-$ ponent may have $U_{\mathrm{H}}$ around $10^{8} \mathrm{~cm} \mathrm{~s}^{-1}$ or possibly less, while the required carbon/hydrogen abundance ratio is larger than it was in a single-component model which sought to explain only the $\mathrm{CIII}] / \mathrm{C} \mathrm{IV} / L \alpha$ ratios. This is the main reason why, for example, a model discussed by Scargle, Caroff, and Tarter (1974) was able to have a carbon abundance significantly larger than the rather low single-component values mentioned by Davidson (1973). Scargle, Caroff, and Tarter postulated a particular, widespread spherical distribution of values of $U_{\mathrm{H}}$. Netzer (1976) later described a somewhat more sophisticated spherical model; while MacAlpine's (1972) earlier calculations had also been spherical. At the level of approximation allowed by available data, these spherical models were practically equivalent to two-component models, with high $U_{\mathrm{H}}$ in the inner regions and low $U_{\mathrm{H}}$ near the outer edges. It has sometimes been said that a two-component model is undesirable because it involves more parameters than, say, a spherical-distribution model; but this is not true, because the latter type of model must have at least one or two parameters which specify the radial dependence of the gas distribution. (These parameters have often been made implicit and therefore inconspicuous, by arbitrarily supposing the gas to have uniform density, in some sense which may involve a constant "filling factor.")

Incidentally, the three sets of spherical models that we have just mentioned exemplify three alternative choices regarding optical depths. Scargle, Caroff, and Tarter (1974) assumed the gas to be optically thin. This simplifies the calculations, but is implausible because of the observed $\mathrm{Mg}$ II emission mentioned earlier, because of the lack of observed Lyman continuum emission from the ionized gas (Davidson, 1976), and because of some photon-counting arguments to be cited in Sec. V. MacAlpine (1972) supposed that the gas is optically thick to ionizing photons, but (mainly for calculative convenience) he assumed that each of the numerous clouds is individually thin. We are not enthusiastic about this picture either-partly because Lyman continuum absorption is usually not observed (Oke, 1974; Osmer and Smith, 1977), partly because the individual clouds would have to be amazingly thin in the geometrical sense (see remarks near the end of Sec. III.B above), and partly because we require large optical depths in the Balmer lines (see Sec. IV.C below). The spherical configuration adopted by Netzer (1976) consisted of many clouds which were each optically thick to ionizing photons; the "covering factor" may be small so that we can see between clouds into the center of activity where the continuum originates.

Next, as discussed by Shields (1976), one can try to estimate the oxygen/nitrogen/carbon abundance ratios by comparing certain faint ultraviolet N III], N IV], OIII ], and OIV] lines with the CIII ] and C IV emission. Shields pointed out that these lines all originate in roughly the same ionization zones, and all have roughly similar temperature dependences; so in some ways their implications are not strongly model dependent. However, the weak lines are too faint to have been reliably measured yet, and some of them may be collisionally deexcited to an uncertain degree (see Baldwin and Netzer, 1978). The most useful weak line is perhaps NIV] $\lambda 1486$, whose ratio with $\operatorname{CIV} \lambda 1549$ is quite a good relative-abundance indicator, and whose critical deexcitation density is several times higher than that of C III ] $\lambda$ 1909. At present there is a suspicion, foreshadowed by Shields' discussion and exemplified in the PHL 938 model described in Sec. III.B, that the nitrogen/carbon ratio is larger than "normal." Where nitrogen is similarly overabundant in some objects in our own Galaxy, it is thought to be due to secondary nucleosynthesis, involving nuclear processing in stellar material which has already been provided with appreciable concentrations of heavy elements. In miscellaneous samples of galactic material, $n_{\mathrm{N}} /\left(n_{\mathrm{C}}+n_{\mathrm{O}}\right)$ is often correlated with $\left(n_{\mathrm{C}}+n_{\mathrm{O}}\right) / n_{\mathrm{H}}$, in a way that has a qualitative explanation involving star formation and nucleosynthesis rates (see Peimbert, 1975; Pagel, 1976; Searle, 1976; Pagel et al., 1978; Shields and Searle, 1978; and references therein). Thus we surmise that an unusually large nitrogen/carbon ratio implies unusually large heavyelement abundances in quasars. But this is only a suspicion, without firm observational or theoretical bases.

What about the form of the ionizing continuum? As explained by Davidson (1977b), if $F(\varepsilon)$ decreases less steeply than $\varepsilon^{-1}$, then a larger carbon abundance and a smaller value of $U_{\mathrm{H}}$ are indicated for the major component of gas. A flatter spectrum, with a high-energy cutoff somewhere beyond $200 \mathrm{eV}$, has some appeal because it makes the high-excitation OVI, NV, and HeII lines easier to explain; in an extreme case, a singlecomponent model might suffice. It is slightly disturbing that there is no evidence for a correlation between observed continuum slopes and line intensities (see Fig. 3 of Baldwin and Netzer, 1978), and in Sec. V we shall worry about whether the observed continua as well as the line intensities may be affected by wavelength-dependent extinction along the line of sight to each quasar.

Occasionally there has been speculation that much of the ionizing radiation in quasars is emitted by some very hot thermal gas $\left(T>10^{5} \mathrm{~K}\right)$. This would be discomfiting, because such gas might produce strong ionizing lines as well as a continuum. For the moment, we shrink from numerical consideration of this possibility_but see Daltabuit, MacAlpine, and Cox (1978).

In summary, the preceding arguments are relevant and necessary, but dissatisfying: the winds of argument blow through too many loopholes, and the field is posted with too many caveats. We can assign limits to the important parameters, but these limits are partly subjective, in the sense that they recede somewhat if one is willing to postulate special circumstances.

How scarce can the heavy elements be? In an early discussion (Davidson, 1973), $n_{\mathrm{C}} / n_{\mathrm{H}}$ in quasars was proposed to be smaller than the solar value by a factor of the order of 6 . But this suggestion followed from "observed" CIII ]/CIV and CIV/L $\alpha$ intensity ratios which were smaller than more recent values, from the use 
of an ionizing continuum which was steeper than currently fashionable continua, from a neglect of collisional deexcitation of CIII ] $\lambda 1909$, and from the use of a one-component model, which seemed acceptable because the OVI and $\mathrm{NV}$ lines were not, at that time, generally known to be intense. Today, the observational data make it difficult to allow a carbon abundance smaller than perhaps half the solar value. A smaller value would not permit the carbon lines to assume their observed share of the cooling.

How overabundant might the heavy elements be? Perhaps several times more abundant than in the Sun, as in the model described in Sec. III.B; but this limit is even more difficult to establish than the lower limit. Imagine, for example, that electron densities are actually as high as $10^{10} \mathrm{~cm}^{-3}$, or even a bit higher; then $\mathrm{C}$ III ] $\lambda 1909$ emission would suffer appreciable collisional deexcitation, requiring a larger carbon abundance to provide the observed intensities. We suspect that one cannot push this speculation very far, because the dependences are such that it would become very difficult to explain why the relative intensities of $\mathrm{C}$ III $\lambda$ 977, C III ] $\lambda 1909$, $\mathrm{CIV} \lambda 1549$, and $L \alpha$ do not vary more from quasar to quasar. It is difficult to make such an argument definite-but given the observed presence of semiforbidden lines in quasars' spectra, and supposing that "God is not malicious," we are prejudiced against special models, in which these lines are severely affected by collisional deexcitation but nevertheless remain prominent because the elemental abundances are carefully adjusted to give compensating effects.

We remark, in passing, that one of the most interesting facts in astronomy is the prevalence of heavy-element abundances which are either like those in the Sun, or scarcer. However, some galactic nuclei may have larger concentrations of heavy elements (see Shields and Searle, 1978). This seems consistent with recent modeling tendencies regarding quasars.

Concerning the ionization parameter $U_{H}$ : Most of the $L \alpha$ emission probably originates in the low $-U_{\mathrm{H}}$ component of gas. There, $U_{\mathrm{H}}$ is of the order of $10^{8} \mathrm{~cm} \mathrm{~s}^{-1}$. This means that the thermal energy density of the ionized gas is between 10 and 100 times the ionizing radiation energy density. This may have some gasdynamical implications, but also, it is something to wonder about: is there a reason for such a factor to occur?

Finally, we close this section with a very large caveat: in Sec. V, we shall explain why much of the above discussion, and some of the observational data, should be taken with a grain of salt, or more likely, with many fine absorbing grains of dust.

\section{LINE TRANSFER}

\section{A. General considerations}

Until now we have supposed that nonionizing photons always escape from nebulae. But this is not necessarily true for "resonance lines" (permitted transitions whose lower levels are ground levels), which can have very large optical depths in a photoionized cloud. Consider, for example, the special case of $L \alpha$. Suppose that its local linewidth is due to the Doppler effect, involving thermal motions of atoms and perhaps subsonic turbulence. In hydrogen gas, the relative linewidth $\Delta \lambda_{L \alpha}$ / $\lambda_{L \alpha}$ is then of the order of $w / c \sim 10^{-4}$ (where $w$ is the speed of sound). Since the oscillator strength for $L \alpha$ is about the same as the integrated oscillator strength for the Lyman continuum, the ratio of $L \alpha$ optical depth to photoionization optical depth is very roughly $\tau_{L_{\alpha}} / \tau_{\mathrm{H}}$ $\sim \lambda_{L \alpha} / \Delta \lambda_{L \alpha} \sim 10^{4}$. Figure 5 shows that $\tau_{\mathrm{H}} \simeq 50$ is easily possible in a photoionized region, giving $\tau_{L \alpha} \sim 10^{6}$ 。 Diffusion of resonance photons through such optical depths is a nontrivial problem.

The first realistic approaches to this problem, so far as we know, were those by Holstein (1947), by Zanstra and by Sobolev (see Zanstra, 1949, 1951). Their results, and the work of Koelblad (1956), Osterbrock (1962), and Capriotti $(1965,1966)$, justify a very simple example, which gives a surprisingly correct impression of the general results. Imagine a simple resonance emission line in a spherical gas cloud. The line opacity is uniform throughout the cloud; the cloud's geometrical radius $r$ corresponds to a line-center optical depth $\tau_{0} \gg 1$. The linewidth is due to thermal motions, so that if we measure the photon wavelength displacement $\eta(\eta=0$ at line center) in appropriate units, then the optical depth profile is simply

$$
\tau(\eta)=\tau_{0} e^{-\eta^{2}}
$$

Suppose that every resonance absorption event is followed by reemission, so that we have pure resonance "scattering." Henyey (1940) pointed out that absorption at $\eta$ leads to reemission at $\eta^{\prime}$ with probability given by a calculable "redistribution function" $R\left(\eta, \eta^{\prime}\right)$. In the thermal-width case, it turns out that we obtain practically correct results by pretending that $\eta^{\prime}$ is uncorrelated with $\eta$-the "complete redistribution approximation." The probability of reemission in interval $d \eta^{\prime}$ then becomes

$$
\phi\left(\eta^{\prime}\right) d \eta^{\prime}=\pi^{-1 / 2} e^{-\eta^{2}} d \eta^{\prime},
$$

regardless of $\eta$. We can also pretend that the scattering is isotropic. Imagine now that a resonance photon is initially created at the center of the spherical cloud. After almost every scattering event, the photon's $|\eta|$ will be small enough so that its mean free path to the next scattering event is not larger than several times $r / \tau_{0} \ll r$. Diffusion in space will require very many scattering events before the photon has traveled appreciably far from the center of the sphere; and before this has happened (as explained by Osterbrock, 1962), there occurs a single scattering event, involving an ion in the "tail" of the Maxwellian velocity distribution, which causes $|\eta|$ to suddenly become large enough so that $\tau(\eta) \lessgtr 1$, allowing the photon to escape "in one jump." Essentially, we can neglect excursions in space (except of course the last one) and consider only excursions in wavelength, each of which is negligibly correlated with previous events. The probability of escape, per scattering event, is approximately

$$
\begin{aligned}
\int \phi(\eta) e^{-\tau(\eta)} d \eta & \approx \pi^{-1 / 2} \int_{-\infty}^{+\infty} \exp \left(-\eta^{2}-\tau_{0} e^{-\eta^{2}}\right) d \eta \\
& \approx \frac{\sqrt{2}}{e \sqrt{\ln \tau_{0}} \tau_{0}} \approx \frac{1}{1.9 \sqrt{\ln \tau_{0}} \tau_{0}} .
\end{aligned}
$$


Most escape events occur near $\eta \simeq \pm \sqrt{\ln \tau_{0}}$. The average number of scattering events before escape is therefore

$$
Q=K_{1} \tau_{0},
$$

where $K_{1} \approx 1.9 \sqrt{\ln \tau_{0}}$ is close to 6 for a wide range of $\tau_{0}$. (Note that when escape occurs after $Q$ scatterings, diffusion in space has typically removed the photon from the center of the sphere by a relatively small distance, only several times $\tau_{0}^{-1 / 2} r$.)

The average distance between scattering events being somewhat larger than $r / \tau_{0}$, the total path length traveled by the photon before escape is

$$
l_{\mathrm{esc}}=K_{2} r \text {, }
$$

where $K_{2}$ is moderately larger than $K_{1}$, and of course the flight time to escape is $l_{\text {esc }} / c$.

If resonance line photons are created throughout the sphere, we find that the appropriate volume-averaged $\bar{K}_{1}$ and $\bar{K}_{2}$ are somewhat less than the central values; and we can use the same equations, crudely, for a plane or cylindrical geometry if $\tau_{0}$ and $r$ are half-thicknesses. These results have several implications:

(1) Density of trapped resonance-line photons: If resonance scattering did not occur, the average escape time would be about $r / c$; with scattering, this is multiplied by $\bar{K}_{2}$. So the equilibrium density of resonance photons is enhanced by a rather modest factor of the order of $\bar{K}_{2}$. Therefore, despite the terrific optical depth for $L \alpha$ in a photoionized region, the radiation pressure of trapped $L \alpha$ photons is not enormously greater than that of the ionizing continuum and may even be smaller.

(2) Continuum absorption of resonance photons: Suppose that resonance photons can be destroyed by some continuum absorption process (e.g., photoionization; or absorption by dust grains) with optical depth $\tau_{c} \ll \tau_{0}$. In the path length $l_{\text {esc }}$, the effective absorption optical depth is $\bar{K}_{2} \tau_{c}$.

(3) Branching alternatives or collisional deexcitation: After an ion has been excited by resonance absorption, it will probably decay by reemitting a resonance photon. But there may be some small probability of alternative decay to an intermediate level, or of ionization of the excited ion, or of collisional deexcitation. Then the resonance scattering process gives $Q$ chances for the alternative process to occur. For example, if the critical electron density for collisional deexcitation of the resonance excited level, neglecting scattering, is $n_{c}$ (typically $10^{15}$ or $10^{16} \mathrm{~cm}^{-3}$ ), then the effective critical density is $n_{c}^{\text {eff }} \simeq n_{c} / Q$.

In principle, these agreeably simple results may seem dangerously oversimplified. The actual redistribution function $R\left(\eta, \eta^{\prime}\right)$ does in fact cause $\eta^{\prime}$ to be somewhat correlated with $\eta$ (Unno, 1952). Worse, for largish $|\eta|$ (typically $|\eta| \gtrless 3$, important for $\tau_{0} \gtrless 10^{4}$ ), the line's wings have a Lorentzian natural profile rather than a Gaussian Doppler profile, so that $\tau(\eta)$ becomes proportional to $\eta^{-2}$ rather than $e^{-\eta^{2}}$, and $\eta^{\prime}$ becomes strongly correlated with $\eta$. Then it turns out that excursions in space as well as in $\eta$ are important. This problem has been solved numerically by Avery and House (1968), Auer (1968), Panagia and Ranieri (1973), and Bonilha et al. (1979), who used Monte Carlo methods, and by Adams (1971, 1972, 1975a, b), Harrington (1973), and Hummer and Kunasz (1979), who used different methods. Their results show, fortuitously but conveniently, that Eqs. (4.4) and (4.5), with moderate values of $\bar{K}_{1}$ and $\bar{K}_{2}$, are indeed realistic for $10^{2} \lessgtr \tau_{0} \lessgtr 10^{7}$. $\bar{K}_{1}$ and $\bar{K}_{2}$ vary only through factors of the order of 3 over this range in $\tau_{0}$. Adams $(1975 b)$ found that $\bar{K}_{2}$ becomes proportional to $\tau_{0}^{1 / 3}$ as $\tau_{0}$ increases beyond $10^{6}$ 。

If $\tau_{0}$ and $r$ in Eqs. (4.4) and (4.5) represent the halfthickness through the appropriate gaseous region, $\bar{K}_{1} \simeq 3$ and $\bar{K}_{2} \simeq 6$ are useful volume-averaged estimates for many practical cases.

We suspect that the Doppler width of a line partly involves local subsonic turbulence; this can appreciably widen a line of a heavy ion species, supplementing the ions' thermal speeds by bulk motions with a fair fraction of the larger sound speed $w$. It is usual to suppose that a turbulent velocity dispersion mimics a Maxwellian distribution (Gaussian in each direction); but there is no assurance that this is correct at the edges of the distribution in $\eta$. In some early discussions, velocity dispersions for line scattering near quasars were taken to be very large because the observed emission lines are so broad; but for various reasons, including analogies with more familiar galactic objects, we now prefer to think that each localized cloud of gas has a relatively small internal velocity dispersion, while an observed linewidth corresponds to relative velocities of thousands of $\mathrm{km} \mathrm{s}^{-1}$ between many different clouds.

Ferland and Netzer (1979) have combined a Monte Carlo resonance scattering program with photoionization calculations and have remarked that it is necessary to be careful with $L \alpha$ and with other resonance lines that partly originate in gas where $n\left(\mathrm{H}^{+}\right) / n\left(\mathrm{H}^{0}\right)$ is not very large. For each resonance line they defined a "generating function" $G\left(\tau_{0}\right)$, proportional to the rate of creation of resonance photon's per unit of optical depth. Some examples of $G\left(\tau_{0}\right)$, for C IV $\lambda 1549$ and $L \alpha$ in typical plane-parallel photoionized regions, are shown in Fig. 8. The generating functions for C IV $\lambda 1549$ are fairly well behaved, so one can easily obtain valid results for this line by using average escape probabilities $Q^{-1}$, estimated from published scattering calculations or guessed from Eq. (4.4). Because there is a limit to the column density of $\mathrm{C}^{3+}$ in a particular model, the $\lambda 1549$ photons can escape through the maximum- $\tau_{0}$ side as well as the

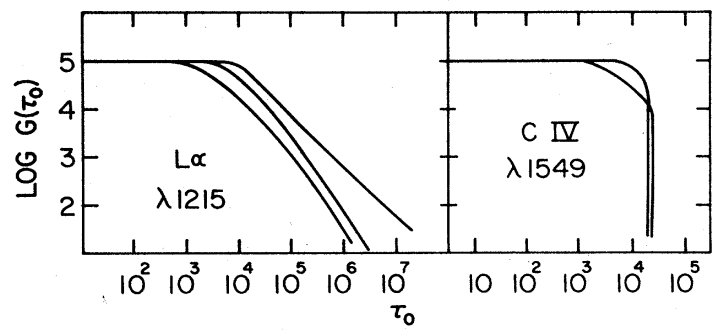

FIG. 8. Generating functions for the $L \alpha$ and C IV $\lambda 1549$ resonance lines in thick photoionized regions, according to Ferland and Netzer (1979). These functions describe the production of resonance-line photons per unit optical depth $\tau_{0}$ at line center. Note that the C IV $\lambda 1549$ production region is distinctly limited in $\tau_{0}$, but collisional excitation in the $\mathrm{H}^{0}$ zone produces some $L \alpha$ emission even for $\tau_{0}>10^{5}$. 
$\tau_{0}=0$ boundary. The cases of O VI $\lambda 1034$ and $N V \lambda 1240$ should be similar.

The $L \alpha$ line is different. Throughout most of the $\mathrm{H}^{+}$ region, for $\tau_{0} \leqslant 10^{4}$ in Fig. $8, L \alpha$ photons result from recombination and $G\left(\tau_{0}\right)$ is automatically rather uniform. But near and beyond the ionization front, where $L \alpha$ emission is caused by collisional excitation of $\mathrm{H}^{0}$ atoms, $G\left(\tau_{0}\right)$ is sensitive to $U_{\mathrm{H}}$ and to the slope of the ionizing continuum. Comparable numbers of $L \alpha$ photons can be created in the two regimes. Ferland and Netzer found that the recombination $L \alpha$ photons cannot penetrate the thick partial- $\mathrm{H}^{0}$ zone, but are reflected back to escape through the $\tau_{0}=0$ boundary. The collisionally excited $L \alpha$ photons also tend to escape in the same direction. A proper representation of $G\left(\tau_{0}\right)$ for $L \alpha$ is important, because two of the processes discussed below (Balmer line emission, and collisional deexcitation of $L \alpha)$ depend upon it.

Minor details: $L \alpha$ connects only the $2 p$ and $1 s$ levels. Collisions with electrons, ions, and atoms cause transitions back and forth between $2 p$ and $2 s$ (Seaton, 1955). Below, when we cite approximate arguments regarding $L \alpha$ scattering, we shall usually ignore the existence of the $2 s$ level, and no serious error will result; but it is understood that the $2 s$ level is included in most authors' detailed calculations. A few other processes, such as induced emission, are similarly glossed over in this discussion, because they are inessential for our purposes.

\section{B. Possible suppression of resonance emission lines}

Now we shall be slightly more specific about some possibilities mentioned above. First, there is the possibility that resonance photons may be absorbed by continuum processes. Absorption by solid grains is potentially so important that we discuss it separately in Sec. V. This leaves ionization of excited ions by the resonance photons. As discussed in Sec. IV.C, the population of $\mathrm{H}^{0}$ atoms in the $n=2$ level is enhanced by $L \alpha$ scattering, and such atoms can be ionized by UV photons with $\lambda<3645 \AA$. Mg II $\lambda 2798$ is the resonance line which is most likely to be destroyed in this way, because the relevant photoionization cross section is largest close to the $3645 \AA$ limit, and also because the excited $\mathrm{H}^{\circ}$ atoms are most numerous in the $\mathrm{H}^{+} / \mathrm{H}^{\circ}$ transition region where the $\mathrm{Mg} I \mathrm{I}$ emission originates. According to Ferland and Netzer (1979), in some extreme models there can be enough excited $\mathrm{H}^{\circ}$ atoms to give a continuum optical thickness of the order of 0.1 near $2798 \AA$. The effective thickness for resonance-scattered photons is enhanced by a factor of the order of $\bar{K}_{2} / 2$, or perhaps $\bar{K}_{2}$ if escape toward the large- $\tau_{0}$ direction is impossible; so it is conceivable that $30 \%$ or more of the $\mathrm{Mg} \mathrm{II} \lambda 2798$ emission might thus disappear.

Another possibility concerns $\mathrm{He}^{0}$ atoms in the metastable $2{ }^{3} S$ level (see Sec. IV.D), which can be photoionized by $\mathrm{CIV} \lambda 1549$ and $L \alpha$ photons. Davidson, Fastie, and Hartig (1977) suggested that $L \alpha$ is reduced this way. But likely column densities of $\mathrm{He}^{0} 2^{3} \mathrm{~S}$ atoms, as estimated by Feldman and MacAlpine (1978) and Netzer (1978), seem too small to destroy much of the CIV or $L \alpha$ emission-as noted by Krolik and McKee (1978). Other excited ions are probably even less important, although we may have overlooked some possibilities.

Next, we mention collisional deexcitation. C IV $\lambda 1549$ is a convenient and important example. The rate for deexcitation of the excited $2 p$ level by thermal electrons is $\left(10^{-7} \mathrm{~cm}^{3} \mathrm{~s}^{-1}\right) n_{e}$ at temperatures near $15000 \mathrm{~K}$. If the radiative decay rate is $A_{j i}$, but $Q$ scattering events must occur before a resonance photon escapes, then the effective radiative rate is $A_{j i} / Q \approx\left(10^{8.4} \mathrm{~s}^{-1}\right) Q^{-1}$. The effective collisional and radiative deexcitation rates are equal at an electron density $n_{c}^{\text {eff }} \approx\left(10^{15.4} \mathrm{~cm}^{-3}\right) Q^{-1}$ 。 To guess $Q$, we need $\tau_{0}$. Fine structure in the upper level splits the $\lambda 1549$ line into two components, and for simplicity we shall use the optical depth in the brighter component. Adopting a thermal linewidth, we find a halfthickness $\tau_{0} \simeq 10^{4}$ in the carbon-rich, $U_{\mathrm{H}} \approx 10^{8} \mathrm{~cm} \mathrm{~s}^{-1}$ model described in Sec. III.B; $\bar{K}_{1} \approx 3$ then gives $Q \approx 10^{4.5}$ and $n_{c}^{\text {eff }} \approx 10^{10.9} \mathrm{~cm}^{-3}$ for $\mathrm{CIV} \lambda 1549$. This value would be decreased for larger $U_{\mathrm{H}}$.

Shuder and MacAlpine (1979) and Ferland and Netzer (1979), considering possible deexcitation of C IV 1549 in more detail, have found that the $\lambda 1549$ intensity is unlikely to be reduced very much except perhaps in extreme, unconventional photoionization models. Ferland and Netzer have argued that the emergent intensity is not strongly reduced even at high densities approaching $n_{c}^{\text {eff }}$, because this line is the primary coolant in regions where $\mathrm{C}^{3+}$ is abundant. If collisional deexcitation of C IV $\lambda 1549$ reduces the cooling efficiency, then the local equilibrium temperature must be increased so that the resulting $\lambda 1549$ intensity is scarcely affected after all. This example shows the danger of adopting any fixed temperature when thinking about this subject. (The result for $\lambda 1549$ becomes more complicated in models with "flat" ionizing continua, where OVI $\lambda 1034$ and $\mathrm{NV} \lambda 1240$ can assume much of the cooling burden.)

Krolik and McKee (1978) and Zirin (1978) suggested that quasars' hydrogen lines are largely "thermalized," by which they meant that collisional excitations and deexcitations from the $n=2$ level are very important. Collisional excitations to $n=3$ are most important, were discussed by Shields (1974) and Netzer (1977a), and will be mentioned in Sec. IV.C below; but here, let us consider collisional deexcitations from the $n=2$ level because these might reduce the $L \alpha$ intensity. The critical electron density, at which collisional deexcitations occur as fast as escaping $L \alpha$ photons, is

$$
n_{c}^{\text {eff }} \approx\left(10^{16.9} \mathrm{~cm}^{-3}\right) Q_{L \alpha}^{-1} \approx\left(10^{16.4} \mathrm{~cm}^{-3}\right) \tau_{L \alpha}^{-1},
$$

where $\tau_{L \alpha}$ is the total line-center optical depth measured from the illuminated boundary (we have assumed that $L \alpha$ photons cannot escape in the direction toward large $\tau_{L \alpha}$ ). In a photoionization model, most of the recombination $L \alpha$ emission comes from zones where $\tau_{L \alpha}<10^{5}$ and therefore is not strongly reduced by collisional deexcitation unless $n_{e}$ exceeds $10^{11} \mathrm{~cm}^{-3}$.

Collisionally excited $L \alpha$ is produced near the ionization front and beyond, where $\tau_{L_{\alpha}}$ becomes very large but where $n_{e}$ is probably smaller than in the $\mathrm{H}^{+}$zones. Where it is produced, collisionally excited $L \alpha$ accounts for most of the cooling, so we have the same effect as for CIV $\lambda$ 1549: any reduction in cooling efficiency simply causes the temperature to rise until the required cooling rate is obtained. The temperature rise in this 
case is only slight; for example, a rise from $T=10000$ $\mathrm{K}$ to $T=12500 \mathrm{~K}$ would increase the basic $\mathrm{H}^{0}$ excitation rate by a factor of 10 . If $n_{e} \gtrless\left(10^{15.5} \mathrm{~cm}^{-3}\right) \tau_{L \alpha}^{-1}$, collisional excitation from the $n=2$ level to higher levels becomes fast enough so that Balmer lines account for a noticeable fraction of the local cooling; in effect, some energy flux is then diverted from $L \alpha$ to the $H \alpha$ line. A photoionization calculation which ignores this effect will nevertheless give almost the correct total for the hydrogen line intensities, provided that the density is not extremely high.

Ferland and Netzer (1979) and Shuder and MacAlpine (1979) have verified some of the above points by including collisional deexcitation in photoionization calculations. The $L \alpha$ output cannot be reduced by more than $30 \%$ or $40 \%$ if $n_{e} \lessgtr 10^{10} \mathrm{~cm}^{-3}$, and the actual effect is probably smaller. $\mathrm{Mg}$ II, Fe II, and C III] emissions may be enhanced by the possible rise in temperature in the $\mathrm{H}^{0}$ zone-unless they also are collisionally deexcited. These effects were included in the calculation discussed in Sec. III.B, but do not amount to much more than the uncertainties in ionic parameters. Often, a photoionization calculation which does not include collisional deexcitation of resonance lines will give fairly correct results for the bright ultraviolet line intensities, even if collisional deexcitation does in fact play a moderate role (this is an example of rules (i) and (ii), cited in Sec. I.D). The fainter lines may be affected, though.

Resonance-excited ions might be photoionized by the incident continuum. For example, if the Balmer continuum $(\varepsilon>3.4 \mathrm{eV}, \lambda<3645 \AA$ ) is intense enough to ionize a hydrogen atom in the $n=2$ level in less time than $Q / A_{j i}$, this would reduce the $L \alpha$ intensity and there would be other effects also. It is easy to show that this process is less important than collisional deexcitation if $U_{\mathrm{H}}$ is less than about $10^{8.7} \mathrm{cms}^{-1}$. However, the effect may provide a small amount of extra heating energy, from the Balmer continuum, in the less-ionized zones.

\section{The hydrogen line spectrum}

In ordinary galactic nebulae, hydrogen emission lines of the visual-wavelength Balmer series ( $H \alpha \lambda 6563$, $H \beta \lambda 4861, \ldots)$ and the infrared Paschen series $(P \alpha \lambda 18751, P \beta \lambda 12818, \ldots)$ are by-products of recombination events. These lines' intensity ratios can be predicted from relative rates of recombination to the various levels, branching ratios of decays toward the ground level, and less important parameters (Seaton, 1959; Brocklehurst, 1971). Radiative transfer does not present much difficulty for ordinary nebulae, which are optically thin in the Balmer and higher series of lines but so thick in the Lyman lines that $L \beta, L \gamma$, etc., are mostly degraded to $L \alpha$ plus other lines (this is called "case B"; see remarks near the end of Sec. II.C). The calculated intensity ratios $I(H \alpha) / I(H \beta)$ and $I(H \beta) / I(H \gamma)$ are rather insensitive to densities and temperature, and generally match observed nebular spectra quite well if certain well-understood observational corrections are made.

However, when broad Balmer lines are observed in the spectra of low-red-shift quasars and Seyfert galaxies, their intensity ratios do not always match the ordinary nebular values: $I(H \alpha) / I(H \beta)$ is often far too large while $I(H \beta) / I(H \gamma)$ is slightly too large (Baldwin, 1975; Weedman, 1977, and references therein; Osterbrock, 1977). Possible explanations have been discussed by many authors, including Osterbrock and Parker (1965), Oke and Sargent (1968), Anderson (1970), Wampler (1971), Adams and Petrosian (1974), and others cited below. Wavelength-dependent extinction by interstellar dust is an obvious possibility - but a reasonable wavelength dependence cannot explain both the $H \alpha / H \beta$ and the $H \beta / H \gamma$ ratios simultaneously. Collisional excitation from the ground level (see Parker, 1964) is also inadequate, even in combination with extinction. Two more promising ideas are that collisional excitation from the $n=2$ level (mentioned near the end of Sec. IV.B) is moderately important, and/or that the emission regions are optically thick in the Balmer lines. These effects may happen if the density of $\mathrm{H}^{0}$ atoms in the $n=2$ level is greatly enhanced by trapped $L \alpha$ photons.

We can guess whether a plane-parallel photoionized region like that described in Sec. III.B is optically thick to Balmer-line photons, as follows. First, let the columnar $L \alpha$ creation rate in the photoionized regioni.e., $L \alpha$ photons created per unit area of illuminated surface-be $\zeta F_{\mathrm{H}}$, where $F_{\mathrm{H}}=n_{e} U_{\mathrm{H}}$ as previously defined and $\zeta$ is of the order of unity. (Realistic models, with $L \alpha$ produced by collisional excitation as well as recombination, usually have $\zeta=1$ to 5 .) Let $Q_{L \alpha}$ denote the average number of scattering events undergone by a $L \alpha$ photon before escape; during each of these "scattering" events, a hydrogen atom lingers in the $2 p$ level for a time $A_{L \alpha}^{-1} \approx 1.6 \times 10^{-9} \mathrm{~s}$. The column density of excited atoms is therefore

$$
N\left(\mathrm{H}^{0} 2 p\right) \approx \zeta F_{\mathrm{H}} Q_{L \alpha} A_{L \alpha}^{-1} \text {. }
$$

According to Eq. (4.4), $Q_{L \alpha} \approx \bar{K}_{1} \tau_{L \alpha}$, where $\tau_{L \alpha}$ is the total line-center optical thickness of the $\mathrm{H}^{+}$region because we suppose that $L \alpha$ photons can escape only through the illuminated boundary. For a thermal linewidth at $T \approx 15000 \mathrm{~K}$, the line-center cross section for $H \alpha$ absorption is $\sigma_{H \alpha} \approx 10^{-12.4} \mathrm{~cm}^{2}$. Thus

$$
\begin{aligned}
\tau_{H \alpha} & \approx \sigma_{H \alpha} A_{L \alpha}^{-1} \zeta F_{\mathrm{H}} \bar{K}_{1} \tau_{L \alpha} \\
& \approx\left(\frac{\zeta F_{\mathrm{H}}}{10^{20.6} \mathrm{~cm}^{-2} \mathrm{~s}^{-1}}\right) \tau_{L \alpha} \\
& \approx\left(\frac{\zeta F_{\mathrm{H}}}{10^{24.4} \mathrm{~cm}^{-2} \mathrm{~s}^{-1}}\right) \tau_{\mathrm{H}} .
\end{aligned}
$$

(Here $\tau_{\mathrm{H}}$ is the photoionization optical depth as previously used.) For a photoionized region like that discussed in Sec. III.B, with $U_{\mathrm{H}} \approx 10^{8} \mathrm{~cm} \mathrm{~s}^{-1}$ and $n_{e} \approx 10^{9.4}$ $\mathrm{cm}^{-3}$, this gives $\tau_{H \alpha} \approx 0.002 \tau_{L \alpha} \approx 20 \tau_{\mathrm{H}}$. The appropriate value of $\tau_{H}$ to limit the region of interest, although indefinite, will exceed unity and may be considerably larger (see Fig. 5); so it is evident that Balmer-line optical thicknesses in quasars' photoionized regions are probably large.

The parameters $\tau_{\mathrm{H}}$ and $\zeta$ are mildly dependent upon $U_{\mathrm{H}}$ and upon the shape of the ionizing continuum, and also, if each linewidth $\Delta \lambda$ is increased, then $\tau_{H \alpha}$ is proportional to $\Delta \lambda^{-2}$. Aside from these dependences, $\tau_{H \alpha}$ is basically a measure of the local photon flux $F_{H}$, which 
probably varies as $R^{-2}$ where $R$ is distance from the hypothetical central source. Since we can roughly estimate a quasar's luminosity, a characteristic value of $F_{H}$ gives us a characteristic radius for the arrangement of Balmer line-emitting regions. This adds interest to an actual estimate of $\tau_{H \alpha}$ derived from line ratios.

Capriotti (1964a, b; 1966) investigated the ratios of optically thick Balmer lines which might occur in lowdensity nebulae $\left(n_{e} \lesssim 10^{5} \mathrm{~cm}^{-3}\right)$; he found that $I(H \alpha) /$ $I(H \beta)$ rose with increasing $\tau_{H \alpha}$, but $I(H \beta) / I(H \gamma)$ decreased. Cox and Mathews (1969) later studied cases with some leakage of Lyman lines. In all of these calculations, absorption processes from levels $n \geqslant 3$ (i.e., Paschen and higher series) were neglected. This was justifiable at low densities, but as the populations of excited levels increase with density, Paschen line absorption can become important near quasars.

Netzer $(1975,1977 a)$ calculated ratios of hydrogen lines at quasarlike gas densities, including absorptions and collisional transitions from many levels; Krolik and McKee (1978), adding some elaborations and considering more extreme cases, later found essentially similar results. These calculations were based upon "mean escape probabilities" for each line, derived from average numbers of scattering events undergone by photons before escape from uniform, plane-parallel slabs (Capriotti, 1965). For recombination lines, Ferland and Netzer (1979) have estimated that the most appropriate "average" value of $\tau_{H \alpha}$, for use in an equation like (4.4), corresponds roughly to the middle of the photoionized region and is smaller than the total $H \alpha$ optical thickness by a factor of 10-30. The effective value of $\tau_{H \alpha}$ for use in conventional quasar-related models is therefore between 50 and 500, accompanied by a $\tau_{L \alpha}$ of the order of $10^{4.7}$. Of course, $\tau_{\mathrm{HB}} \approx 0.2 \tau_{H \alpha}$, $\tau_{H \gamma} \approx 0.06 \tau_{H \alpha}$, etc.

In a gas which is optically thick in the Balmer lines, an obvious effect is the conversion of $H \beta$ to $P \alpha$ and $H \alpha$, because after every $H \beta$ absorption event, the hydrogen atom which is left in the $n=4$ level has a $52 \%$ probability of decaying to $n=3$ (emitting a $P \alpha$ photon) rather than to $n=2(H \beta)$. This increases $I(H \alpha) / I(H \beta)$, and since $\tau_{H \gamma}<\tau_{H \beta}, I(H \beta) / I(H \gamma)$ is decreased. But as Netzer (1975) pointed out, the Paschen optical depth $\tau_{P \alpha}$ may become significant if $\tau_{H \alpha}$ is sufficiently large, because trapped $H \alpha$ photons increase the $n=3$ population. If $P \alpha$ photons cannot escape freely, the emergent $H \beta$ intensity is increased at the expense of $P \alpha$ and $H \alpha$. Figure 9 shows typical behavior of emergent $H \alpha, H \beta, H \gamma$, and $P \alpha$ intensities in cases where these lines result almost entirely from recombination; if the effective value of $\tau_{H \alpha}$ is of the order of 100 -as indeed it should be, in photoionization models with $10^{9}<n_{e}<10^{10} \mathrm{~cm}^{-3}$-then the effective value of $\tau_{P \alpha}$ is of the order of 2 and $I(H \alpha) /$ $I(H \beta) / I(H \gamma) \sim 5 / 1 / 0.35$. These line ratios are just like those observed in typical quasars and Seyfert galaxies.

Some of the Balmer and Paschen emission may result from thermal collisional excitations of $\mathrm{H}^{0}$ atoms. Such excitations directly from the ground level are negligible with ionizing continua like $F(\varepsilon) \sim \varepsilon^{-1}$ (Netzer, 1977a), but may be important for "flatter" or "harder" continua, whose soft $x$ rays cause more heating beyond the $\mathrm{H}^{+} / \mathrm{H}^{0}$

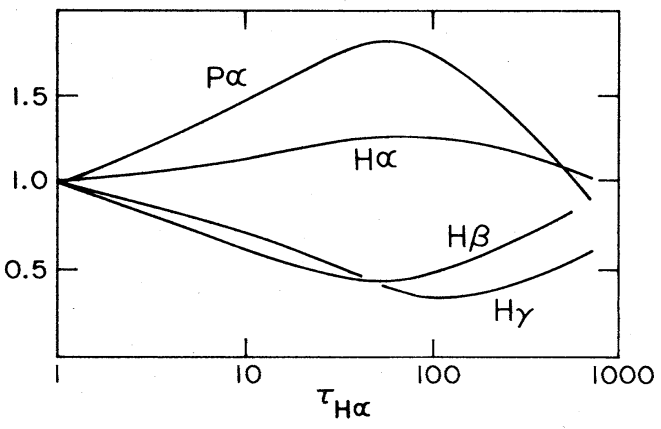

FIG. 9. Schematic dependences of the $H \alpha, H \beta, H \gamma$, and $P \alpha$ recombination line intensities upon $\tau_{H \alpha}$, the optical depth in the $H \alpha$ line. Each ordinate is roughly a line intensity relative to the traditional nebular "Case B" value. Possible contributions due to collisional excitation of $H^{0}$ are not included here.

ionization front. More generally important at high densities and moderate temperatures are excitations from the $n=2$ level, as mentioned by Shields (1974), Netzer (1977), Krolik and McKee (1978), and Ferland and Netzer (1979). Such excitations are important if the electron density exceeds $10^{9} \mathrm{~cm}^{-3}$. However, the process cannot be calculated very reliably, because it depends upon the $n=2$ population. According to Ferland and Netzer, hydrogen lines tend to result from recombination where $\mathrm{H}^{+}$predominates, and from collisional excitation where $\mathrm{H}^{0}$ predominates; to some extent the two regions may be considered separately. A homogeneous, uniform-temperature model is generally not a valid approximation to this type of situation.

The collisional excitations mentioned above are caused by thermal electrons. But in a case where soft-x-ray photons are numerous enough, and in a region where $n_{e} \ll n\left(\mathrm{H}^{0}\right)$, additional effects may be caused by nonthermal electrons with energies of the order of $100 \mathrm{eV}$. Such electrons are produced by soft- $\mathrm{x}$ - ray photoionizations (especially of helium atoms), and may lose appreciable fractions of their initial energies by ionizing and exciting $\mathrm{H}^{0}$ and $\mathrm{He}^{\circ}$ (Spitzer and Tomasko, 1968; Spitzer and Scott, 1969; Dalgarno and McCray, 1972; Shull, 1979). This effect is an exception to the rule that elastic collisions usually cause effectively instantaneous thermalization of most superthermal particles in nebular situations. Direct excitation of lines in this manner has not been included in most photoionization calculations.

In summary, the observed intensity ratios of the Balmer and Paschen lines in quasars' spectra seem to be quite reasonable for photoionized regions with densities $n_{e} \gtrless 10^{9} \mathrm{~cm}^{-3}$, but theoretical complexities presently deter us from making very specific additional statements. There are observational difficulties, too, including the possibility that wavelength-dependent extinction may occur outside the emission regions (see Sec. V).

The various Balmer and Paschen line profiles may be different, if each line preferentially originates in a different part of the gas because of the interplay of all the effects described above. Such differences in line shapes would not be large and have not yet been established. In objects whose lines vary in time, one can look for 
changes in the intensity ratios in order to check their consistency with possible changes in $\tau_{H \alpha}$ (see Boksenberg and Netzer, 1977; Tohline and Osterbrock, 1976; Phillips, 1978a).

It is essential to remember that each detailed calculation, such as we have been mentioning, refers only to a small region (cloud?) of gas, while a real quasar's spectrum must be the total of many such localized spectra. The distributions of the gas, in density as well as location, are indeterminate. This fact helps to cause "observational entropy," which leads to the second rule cited in Sec. I.D.

Finally, we should mention the $L \beta$ line, which for reasons mentioned above must be faint. The presence or absence of $L \beta \lambda 1026$ in a quasar's spectrum is very difficult to establish, because of the usual presence of OVI $\lambda 1034$ emission. Bahcall (1966) discussed the possible escape of $L \beta$ emission from quasars' clouds; Adams (1975a) and Netzer (1976) later considered the problem with some refinements and similar results. It appears that $\tau_{L_{\beta}} \lesssim 10$ would be required in order to permit $I(L \beta) / I(L \alpha) \gtrsim 0.1$. Such a small optical depth is possible only if the local velocity dispersion (i.e., in each small fraction of each ionized zone) is very large (thousands of $\mathrm{km} \mathrm{s}^{-1}$ ). As remarked by Netzer (1976), the $L \gamma$ line would then be almost half as bright as $L \beta$; and $L \gamma$ has not been observed. Netzer also discussed the possibility that $L \beta$ can be enhanced in clouds with more conventional internal velocity dispersions, if $\tau_{H \alpha}$ is large; but even in the most favorable cases $I(L \beta) / I(L \alpha)$ $\leqslant 0.05$. Because the $O$ VI $\lambda 1034$ line is brighter, and because rest wavelengths shortward of $L \alpha$ are observationally difficult, the $L \beta$ line is of little use at the present time.

\section{The helium line spectrum}

Several helium lines-HeI $\lambda \lambda 4471,5876,7065$, HeII $\lambda \lambda 1640,4686$ - have been observed in quasars' spectra and may be used to estimate the helium abundances there. The He $\amalg$ lines may also provide information about possible wavelength-dependent extinction, while the He I line ratios give us some hints about radiation densities in the line-emitting regions.

The He II lines are mainly due to recombination and should not be significantly affected by self-absorption; their standard "case B" emissivities, as used for galactic nebulae (Seaton, 1959; Brocklehurst, 1971) but extended to higher densities, are probably adequate. ${ }^{7}$ These lines provide an indication of the number of $\mathrm{He}^{+}$ionizing photons $(\varepsilon>54.4 \mathrm{eV})$ which are absorbed in the emission regions (Williams, 1971). Moreover, the intrinsic ratio $I(\lambda 1640) / I(\lambda 4686)$ should be close to 7

\footnotetext{
${ }^{7}$ Chan and Burbidge (1975) sugge sted that $L \alpha \lambda 1215$ depopulates the $n=4$ level of $\mathrm{He}^{+}$by causing stimulated emission. This would reduce the HeII $\lambda 4686$ intensity. However, as remarked by Davidson (1977), even if the wavelength coincidence were perfect, the necessary $L \alpha$ density would require unlikely gas densities $\left(n_{e}>10^{11} \mathrm{~cm}^{-3}\right)$; and Krolik and McKee (1978) have doubted that the effect would work even at high densities. Moreover, the relevant wavelengths (hydrogen $L \alpha$, and the $H \beta$ analog of $\mathrm{He}^{+}$) differ by $0.5 \AA$, which is equivalent to a velocity difference of more than $100 \mathrm{~km} \mathrm{~s}^{-1}$.
}

or 8 , with only slight dependence on temperature or density; this fact should be useful in assessing the wavelength-dependent influence of intervening material upon quasars' spectra. In some low-red-shift quasars, the $\lambda 4686$ line is surprisingly faint (Bahcall and Oke, 1971; Baldwin, 1975; Chan and Burbidge, 1975).

The HeI lines are perhaps more interesting, because they represent a larger fraction of the helium and also because they are subject to complicated transfer effects. Two types of helium recombination lines are observed in ordinary nebulae: those resulting from transitions between singlet levels, and those involving triplet levels. We shall not discuss the singlet lines, because they are relatively faint and should behave qualitatively like hydrogen lines. The triplet lines are different, because the lowest triplet level $\left(1 s 2 s^{3} S\right.$ or $2{ }^{3} S$ ) is metastable; forbidden decay from there to the ground level $\left({ }^{1} S\right)$ usually requires a long time, about $8000 \mathrm{~s}$ (Griem, 1969; Drake and Dalgarno, 1969; Drake, 1971). At large densities $\left(n_{e} \gtrsim 10^{4} \mathrm{~cm}^{-3}\right), 2{ }^{3} S$ is depopulated mainly by collisionally induced transitions to $2{ }^{1} S$ and $2{ }^{1} \mathrm{P}$. The equilibrium population of the metastable level can be large enough to cause optical depth effects.

Figure 10 shows the lowest $\mathrm{He}^{0}$ triplet levels. Some galactic nebulae are optically thick in the $\lambda 3889$ line, so that decays from $3{ }^{3} \mathrm{P}$ to $3^{3} \mathrm{~S}$ convert some trapped $\lambda 3889$ photons to $\lambda \lambda 43000,7065,10830$ emission. Observed relative intensities of $\lambda 3889, \lambda 7065$, and other lines then provide enough information to estimate $\tau(\lambda 3889)$ and to make appropriate allowances when deducing the helium abundance; see Robbins (1968, 1970), Robbins and Robinson (1971), and Brocklehurst (1972). Such nebulae are also thick in the $\lambda 10830$ line.

However-as the reader will probably not be surprised to learn-additional complications occur near quasars. Many quasars' and Seyfert galaxies' HeI $\lambda 5876$ intensities, when compared with $H \alpha$ or $H \beta$, imply larger-than-"normal" helium abundances $\left(n_{\mathrm{He}} / n_{\mathrm{H}} \gtrless 0.2\right)$ if ordinary nebular assumptions are made (Baldwin, 1975; Osterbrock, 1977). If some of the hydrogen emission is collisionally excited, as discussed in Sec. IV.C, the results become more extreme. Today it is believed that the helium is not greatly overabundant, but rather that a quasar's $\lambda 5876$ emission is enhanced by processes that are negligible in lower-density nebulae.

Shields, Oke, and Sargent (1972) and Shields (1974) pointed out that soft-x-ray photoionization is more effective for helium than for hydrogen, so that helium/

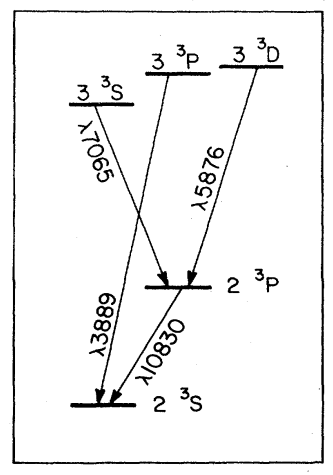

FIG. 10. The lowest triplet levels of $\mathrm{He}^{0}$. 
hydrogen recombination line ratios can be misleadingly large in $\mathrm{H}^{0}$ regions excited by soft $\mathrm{x}$ rays. But this process would enhance most of the helium lines togeth$\mathrm{er}$, and in particular, the HeI $\lambda 4471$ line $\left(2{ }^{3} P-4{ }^{3} D\right)$ should have almost $40 \%$ as large an intensity as $\lambda 5876$ if both are straightforwardly due to recombinations. Although the $\lambda 4471$ line is too faint to observe accurately in most quasars, it appears that $I(\lambda 4471) /$ $I(\lambda 5876)<0.3$ in some cases (Baldwin, 1975). Therefore selective enhancement of $\lambda 5876$ seems to be required.

Mac Alpine (1976) proposed collisional excitation as a suitable mechanism for enhancing $\lambda 5876$. Collisional excitation of ground-level helium atoms by thermal electrons is almost certainly negligible, while collisional excitation from the metastable $2{ }^{3} S$ level to $3{ }^{3} \mathrm{D}$ (see Fig. 10) is expected to have a small rate coefficient. Therefore MacAlpine suggested that the excitations are based on the $2^{3} P$ level. If the re are enough metastable $\mathrm{He}^{0} 2{ }^{3} \mathrm{~S}$ atoms, the gas is optically thick in the $\lambda 10830$ line; trapped $\lambda 10830$ photons then enhance the $2{ }^{3} P$ population and the rate for $2{ }^{3} P-3{ }^{3} D$ excitation by electron collisions is appreciable. In order to obtain the desired enhancement of $\lambda 5876$, MacAlpine invoked a trapping factor (number of scatterings before escape) $Q(\lambda 10830) \sim 300$, optical depth $\tau(\lambda 10830) \sim 100$, and electron density $n_{e} \sim 10^{9.4} \mathrm{~cm}^{-3}$. He remarked that the enhancement factor can give information about the gas distribution, because it depends upon the local flux parameter $F_{\mathrm{H}}=n_{e} U_{\mathrm{H}}$. Feldman and MacAlpine (1978) have made detailed calculations involving 11 levels of $\mathrm{He}^{\mathrm{o}}$, with particular reference to the spectra of certain Seyfert galaxies, and have essentially verified MacAlpine's earlier estimates. Netzer (1978) incorporated multilevel helium into specific photoionization calculations.

The enhancement of He I $\lambda 5876$ is more complicated than that of $H \alpha$, but has similar results: The enhancement factor depends upon the thickness of the $\mathrm{He}^{+}$zone in such a way as to be dependent upon $F_{H}$ and less sensitive to $n_{e}$. One can use photoionization models to guess $U_{\mathrm{H}}$ and therefore to assign a characteristic value of $n_{e}$ to any particular $F_{\mathrm{H}}$. The slope of the ionizing continuum is important; for a given value of $F_{\mathrm{H}}, \lambda 5876$ is enhanced more strongly if there are relatively more higher-energy photons.

In order to enhance HeI $\lambda 5876$ by a factor of 2 , which would roughly match observations, $F_{H}$ must be almost $10^{18} \mathrm{~cm}^{-2} \mathrm{~s}^{-1}$. With reasonable values for $U_{\mathrm{H}}$, this implies $n_{e} \sim$ several times $10^{9} \mathrm{~cm}^{-3}$. These values are like the photoionization model in Sec. III.B and are also consistent with the Balmer line ratios as discussed in Sec. IV.C; the results of Feldman and MacAlpine (1978) and Netzer (1978) seem reasonably consistent. The proposed value of $F_{\mathrm{H}}$ would occur about 10 or 20 lightyears from the center of a luminous quasar, or less than 1 light-year from the center of a rather modest Seyfert nucleus. The helium abundance is probably close to "normal," i.e., $n_{\mathrm{He}} / n_{\mathrm{H}} \approx 0.1$, not far above the value produced in the primordial fireball. But this statement is not very certain, and we should acknowledge that some of the above-cited numerical results are not valid in a speculative model that we discuss in Sec. V.F below.
Feldman (1978) has shown that if $U_{\mathrm{H}} \gtrsim 10^{9} \mathrm{~cm} \mathrm{~s}^{-1}$, then the $\mathrm{He}^{0} 2{ }^{3} S$ and $2{ }^{3} P$ levels are noticeably depopulated by photoionization. This tends to reduce $\tau(\lambda 10830)$.

The HeI $\lambda 5876$ line is broader than $H \beta$ in some spectra (see references cited by Netzer, 1978). Netzer has argued that the $\lambda 5876$ emission comes preferentially from regions with larger $F_{\mathrm{H}}$, i.e., from regions closer to the center, as discussed above; so that the difference in linewidths means that velocity dispersions increase inward. This would occur in some types of gravitationally bound systems.

\section{E. Fluorescent processes}

Suppose that an ion species $\mathrm{Z}^{z_{+}}$has a resonance line whose wavelength nearly coincides with a strong emission line produced by some other ion species, so that $\mathrm{Z}^{z+}$ ions are radiatively excited by the emission line. Suppose that the $\mathrm{Z}^{z+}$ ions also have intermediate levels, which provide alternative routes for radiative decay from the radiatively excited level. Then resonance fluorescence occurs: the strong emission line is partly converted to longer-wavelength lines of $\mathrm{Z}^{z+}$.

The classic nebular example involves some $O$ III lines emitted in transitions between several $2 p 3 d, 2 p 3 p$, and $2 p 3 s$ levels, which are about 41,37 , and $33 \mathrm{eV}$ above the $2 p^{2}{ }^{3} \mathrm{P}$ ground level of $\mathrm{O}^{++}$. These spectral lines have wavelengths between 2800 and $3800 \AA$. In nebulae, they should not be significantly produced either by collisional excitation (the excitation energies are too high) or by recombination (oxygen is much scarcer than hydrogen and helium); nevertheless, the lines are observed in many nebular spectra. This was explained long ago by Bowen $(1934,1935)$, as follows: The $\mathrm{He}^{+}$ analog of $L \alpha(\lambda=303.79 \AA)$, which is produced strongly by recombination, can excite $\mathrm{O}^{++}$from its $2 p^{2}{ }^{3} P_{2}$ nearground level to $2 p 3 d^{3} P_{2}^{0}(\lambda=303.80 \AA)$. From this excited level, an $\mathrm{O}^{++}$ion can decay through the other levels mentioned above, producing the observed lines. The Bowen process is numerically complicated, because the HeII $\lambda 304$ recombination photons must coexist with $\mathrm{O}^{++}$ions, and these resonance photons can also cause photoionization of $\mathrm{H}^{\circ}, \mathrm{He}^{\circ}$, and other species. Asecondary process is also possible: $\mathrm{O}$ III $\lambda 374$ photons, emitted in transitions downward from the $2 p 3 s{ }^{3} P^{0}$ level, may be absorbed by $\mathrm{N}^{++}$to give additional fluorescent lines including $\mathrm{N}$ III $\lambda 4640$.

Weymann and Williams (1969) calculated relevant conversion efficiencies and escape probabilities for HeII $L \alpha$ in regions ionized by moderate power-law continua like $F(\varepsilon) \sim \varepsilon^{-1.2}$. Their calculations, although intended to represent forbidden-line gas in Seyfert galaxies, are probably relevant to higher-density gas. Assuming thermal linewidths, they found large efficiencies $(20 \%-45 \%)$ for Bowen conversion of He II $L \alpha$ to OII emission; but they also pointed out that velocity gradients can be important. At present, the Bowen O III emission must be regarded as only a possible indicator of internal motions in quasars' clouds; observational data are too limited for any significant conclusions.

Perhaps more useful are the OI $\lambda \lambda 1304,8446$ lines. In many Seyfert galaxies and quasars, $I(\lambda 8446)$ $\sim 0.05 I(H \alpha)$ (Oke and Shields, 1976; Netzer and Penston, 
1976; Phillips, 1978); and in the spectra of some highred-shift quasars, $I(\lambda 1304) \sim 0.05 I(L \alpha)$ (Osmer and Smith, 1976, 1977; Baldwin and Netzer, 1978). These lines cannot result from collisional excitation or from recombination, because the excitation energies are high, because oxygen is expected to be too scarce, and because certain other OI lines are not seen. The most likely excitation mechanism is that described by Bowen (1947), who noted a near-coincidence between $L \beta$, at $\lambda=1025.72 \AA$, and the $1025.77 \AA$ transition between atomic oxygen levels $2 p^{4}{ }^{3} P_{2}$ (practically ground level) and $2 p^{3} 3 d^{3} D^{0}$. If an oxygen atom is radiatively excited by $L \beta$, it can decay to $2 p^{3} 3 p{ }^{3} P(\lambda=11290 \AA)$, then to $2 p^{3} 3 s^{3} S^{0}(8446 \AA)$, then to the ground level (1304 $\AA$ ). This process is not very effective in ordinary galactic nebulae, where $L \beta$ photons are too easily destroyed by conversion to $H \alpha$ and $L \alpha$. But Shields (1974) pointed out that a large optical thickness $\tau_{H \alpha}$, as discussed above for quasars and Seyfert galaxies, can increase the $L \beta$ density so that resonance excitation of oxygen becomes more effective. Oke and Shields (1976) suggested that the OI $\lambda 8446$ emission in Seyferts may involve either this process, or else radiative excitation by the continuum, which occurs in galactic nebulae (Grandi, 1975). However, the latter process should also form OI $\lambda 7990$ emission, which is not observed inquasars and Seyferts (but see Christensen, 1979). The OI $\lambda \lambda 1304,8446$ lines probably confirm that Balmerline optical depths are large as discussed in Sec. IV.C.

Netzer and Penston (1976) estimated the OI $\lambda 8446$ production efficiency, using mean escape probabilities with uniform distributions of atomic oxygen and $\tau_{H \alpha}$; they found that

$$
\frac{I(\lambda 8446)}{I(H \alpha)} \simeq 0.04 Q_{H \alpha} \frac{n_{\mathrm{O}}}{n_{\mathrm{H}}},
$$

where $Q_{H \alpha}^{-1}$ is a mean escape probability. $Q_{H \alpha}$ is proportional to $\tau_{H \alpha}$, and, according to Sec. IV.C above, is probably of the order of $10^{3}$ in quasars' broad-line emission regions. This gives $I(\lambda 8446) \sim 0.02 I(H \alpha)$ if the oxygen abundance is conventional (solar); but heavy elements like oxygen may be "overabundant" near quasars (see Sec. III). Hence the observed $\lambda 8446$ intensities are explainable without much difficulty. The main limitation of the calculations is the assumption of uniformity, but the orders of magnitude are probably correct. The OI lines provide gratifying confirmation of Secs. III.C and III.D; it now seems probable that relevant electron densities are mostly close to $10^{9.5} \mathrm{~cm}^{-3}, U_{\mathrm{H}}$ is around $10^{8} \mathrm{~cm} \mathrm{~s}^{-1}$, the gas is optically thick in many hydrogen and helium lines, etc.

We shall mention OI $\lambda \lambda 1304,8446$ again in Sec. V, because these lines may be useful indicators of "reddening" or wavelength-dependent extinction by interstellar dust grains. The emission of an OI $\lambda 8446$ photon is almost inevitably followed by emission of a $\lambda 1304$ photon, giving an intrinsic ratio $I(\lambda 1304) /(\lambda 8446) \simeq 6.5$. If the observed ratio is smaller, then there must be preferential extinction of the $\lambda 1304$ line.

$\mathrm{Mg} I \mathrm{II} 2798$ is prominent in quasars' spectra. Grandi and Phillips (1978) have proposed that a feature near $2950 \AA$ is $\mathrm{Mg} \amalg \lambda 2934$, and that both this and at least part of $\lambda 2798$ are fluorescence lines excited by $L \beta$,
NV $\lambda 1240$, or both. There are serious difficulties with $L \beta$ fluorescence: it should entail other emission lines which are not seen, the observed ratio $I(\mathrm{Mg} I \mathrm{II} 2934) /$ $I(\mathrm{O} I \lambda 8446)$ would require an implausibly large magnesium/oxygen abundance ratio, and (because the relevant wavelength coincidence is imperfect) implausibly large local turbulent velocities of $100 \mathrm{~km} \mathrm{~s}^{-1}$ would be required. Since the wavelength coincidence involving NV $\lambda 1240$ is even less perfect, velocity differences of several hundred $\mathrm{km} \mathrm{s}^{-1}$ would be required for excitation by $\lambda 1240$; but Grandi and Phillips suggest that in this case, the velocity differences need notbe localthe $\mathrm{NV}$ emission may be emitted and absorbed in different clouds of gas. The line near $2950 \AA$ has roughly a fourth the intensity of $\mathrm{MgII} \lambda 2798$, and merits further investigation; its wavelength does not straightforwardly match $\lambda 2934$.

\section{DUST AND QUASARS}

\section{A. Correlation of visual and ultraviolet data}

In 1976 or 1977 , it became evident that something important was missing from previous analyses of quasars' spectra. Ultraviolet lines with rest wavelengths $<2000 \AA$ had been measured only in high-red-shift spectra, while visual and infrared lines with $\lambda>3000 \AA$ had been measured only for low-red-shift objects. In most attempts to specify composite sets of line intensities, the visual and ultraviolet spectra had been joined by supposing that $I(L \alpha) / I(H \beta)$ was of the order of 40 as in the simplest photoionization models. However, Baldwin (1977a) showed evidence for a much smaller $L \alpha / H \beta$ ratio. Assuming that low- and high-red-shift quasars are basically alike, he constructed a composite spectrum in which the connection near $2500 \AA$ was made by referring to the continuum brightness and to the $\operatorname{Mg} I \lambda 2798$ line. This led to $I(L \alpha) / I(H \beta) \approx 3$, which has since been confirmed-except perhaps for an inessential factor of 2 or so-by ultraviolet and infrared observations. Wu(1977), Davidsen, Hartig, and Fastie (1977), Boksenberg et al. (1978), and others have used rocket-and satellite-borne instruments to observe ultraviolet spectra of objects whose visual-wavelength spectra are known from ground-based observations. Hyland, Becklin, and Neugebauer (1978), Soifer et al. (1979), and Puetter, Smith and Willner (1979) have observed visual-rest-wavelength lines shifted into the infrared in high-red-shift spectra. In each case, $I(L \alpha) /$ $I(H \beta)$ is not far from 5 or 6 while $I(L \alpha) / I(H \alpha)$ is less than 2. Thus we have the so-called " $L \alpha / H \beta$ (or $L \alpha / H \alpha$ ) problem": the observed ratios are an order of magnitude smaller than the expected values.

In Sec. IV we have already mentioned one possible explanation, proposed by Krolik and McKee (1978) and by Zirin (1978), wherein collisional excitations and deexcitations from $n=2$ enhance the Balmer lines while reducing $L \alpha$. But if the observed $L \alpha / H \alpha / H \beta$ ratios are to be explained entirely in this way, the electron densities must be above $10^{10} \mathrm{~cm}^{-3}$, making the observed C III] $\lambda 1909$ emission an embarrassment.

Moreover, other ultraviolet/visual line ratios also show discrepancies. The HeII $\lambda 1640$ and $\lambda 4686$ recombination lines are analogous to the $H \alpha$ and $P \alpha$ lines 


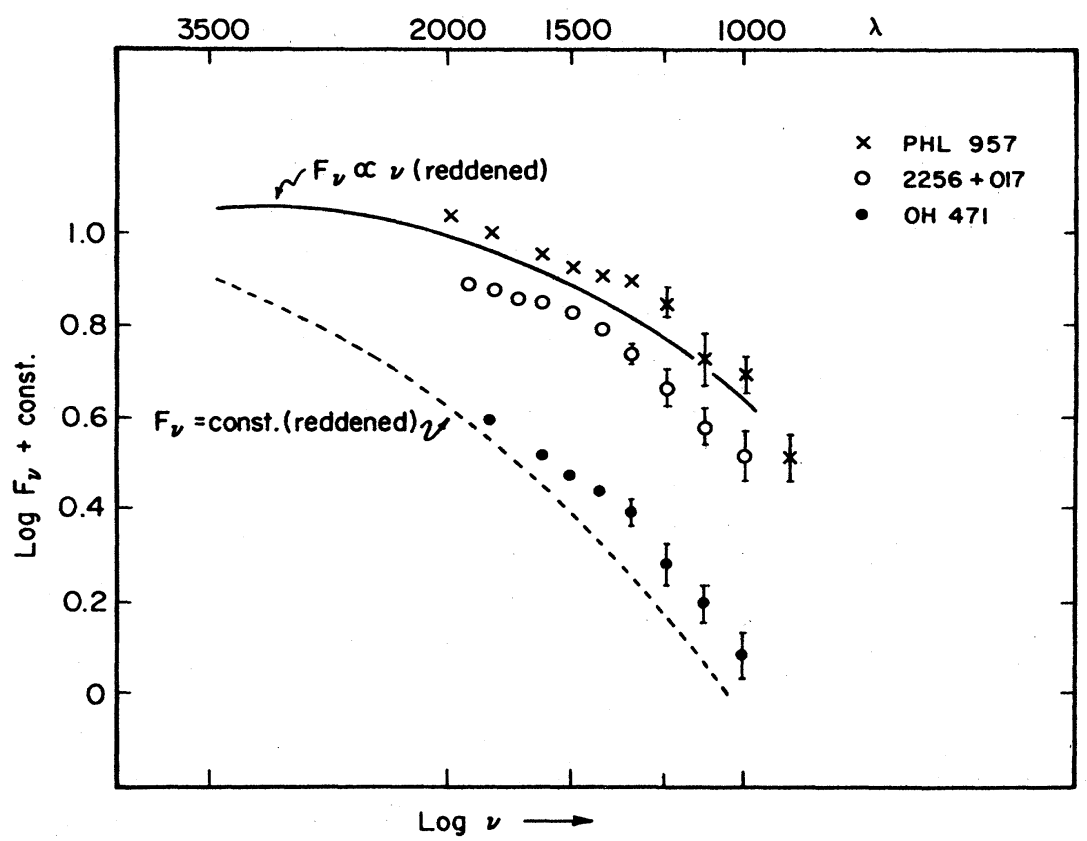

FIG. 11. Quasars' ultraviolet continua as reddened power laws. The crosses, circles, and dots show the apparent continua of three high-redshift quasars, at ultraviolet rest wavelengths. The curves show two hypothetical continua which initially were power laws (flat or rising), but which have suffered rather ordinary amounts of interstellar reddening by dust grains without a $2200 \AA$ feature. The point of this figure is that the $\log -\log$ curvatures of the reddened power laws resemble the curvatures of the observed continua. of hydrogen, but require far higher densities than the hydrogen lines in order to be affected by self-absorption and collisional excitations; $I(\lambda 1640) / I(\lambda 4686)$ should be close to 7 under plausible conditions. But it appears that $I(\lambda 1640) / I(L \alpha) \approx 0.05$ in typical high-red-shift quasars while $I(\lambda 4686) / I(H \beta) \approx 0.15$ in low-red-shift quasars (e.g., see Baldwin, 1975; Baldwin and Netzer, 1978), giving $[I(\lambda 1640) / I(\lambda 4786)]_{\text {apparent }} \approx 2$ if the apparent $L \alpha /$ $H \beta$ ratio is 6. (Cf. Netzer and Davidson, 1979; Shuder and MacAlpine, 1979). Observations by Osterbrock and Koski (1976) and Boksenberg et al. (1978), when combined, give the same result for the particular Seyfert galaxy NGC 4151. Another pair of useful lines is OI $\lambda 1304$ and $\lambda 8446$, mentioned in Sec. IV.E, whose intrinsic ratio should be $I(\lambda 1304) / I(\lambda 8446) \approx 6.5 .^{8}$ Again making a composite estimate, using references cited in Sec. IV.E, we find that $[I(\lambda 1304) / I(\lambda 8446)]_{\text {apparent }}$ is closer to unity than to 6.5 . For the quasar $3 \mathrm{C} 273$ this ratio may be less than unity, although the $\lambda 1304$ intensity is very uncertain (Oke and Shields, 1976; Boksenberg et al., 1978). Therefore, although the HeII and $O$ I lines are faint and difficult to measure, we think that they provide evidence for an overall ultraviolet/ visual discrepancy, a factor of the order of 4 which probably also accounts for much of the $L \alpha / H \beta$ discrepancy.

This ultraviolet/visual deficiency, if real, is evidence that many small solid grains exist somewhere along the line of sight to each quasar. Interstellar dust grains in our Galaxy are known to "redden" the light of distant stars, i.e., they absorb and scatter more at shorter wavelengths. Similar reddening has long been suspected for Seyfert galaxies, partly because of certain [SII] in-

${ }^{8} \mathrm{O}$ I $\lambda 1304$ is a resonance line, but resonance scattering and collisional deexcitation should affect it less than in the case of $L \alpha$, because oxygen is far less abundant than hydrogen and the refore has smaller optical depths. frared/visual line ratios (see Wampler, 1968, 1971; Rees et al., 1969; Osterbrock, 1978). If quasars' emission-line spectra are reddened by intervening dust, their continua are probably also affected. Continuum reddening and line reddening can be different, ${ }^{9}$ but for simplicity we shall assume that they are the same. One argument against reddening of quasars, based on assumed power-law spectra (McKee and Petrosian, 1974), is not very strong because the observed continua are not smooth power laws (see Fig. 1). A broad absorption feature near $2200 \AA$, often associated with interstellar reddening in our Galaxy, is not prominent in quasars; but this does not disprove reddening there, because the physical basis of this feature is not well understood, and besides, a somewhat mysterious "bump" around $3000 \AA$ makes the 2000-4000 $\AA$ continua uncertain for many quasars. If we don't worry about these features, we can fit the observed shapes of typical high-red-shift continua by smoothly reddened power laws - with the important detail that the intrinsic continua $F(\varepsilon) \sim \varepsilon^{-s}$ may have spectral indices $s$ which are zero or negative (Fig. 11).

$P \alpha$ emission has been measured in at least two quasars' spectra (Grasdalen, 1976; Puetter et al., 1978). Since this line has the same upper level as $H \beta$, it has been suggested that the apparent $P \alpha / H \beta$ intensity ratio can be used to measure reddening. The indicated amount of reddening is less than we have suggested in the case

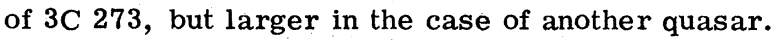
However, as explained in Sec. IV.C, the Balmer and Paschen lines of quasars are probably affected by selfabsorption effects, and also, an infrared/visual red-

\footnotetext{
${ }^{9}$ For example, suppose that reddening occurs near a quasar; imagine a model wherein the line-emitting regions are distributed in a doughnut-shaped volume largely surrounded by dust, but with less dust covering the "hole of the doughnut" where the compact continuum source is.
} 
dening deduction, e.g., from $P \alpha / H \beta$, must be extrapolated to give the much stronger ultraviolet effect. Therefore we shall not weight the $P \alpha / H \beta$ results very highly in this discussion.

There are several possible locales for the hypothetical dust grains. We shall now discuss these locales separately.

\section{B. Dust in our Galaxy}

Dust grains (solid particles with sizes $<1 \mu \mathrm{m}$; "smoke" might have been a better term) are associated with interstellar gas almost everywhere in our Galaxy. The investigation of such dust has become a sizable topic: see the review articles by Wickramasinghe and Nandy (1972), Aannestad and Purcell (1973), Salpeter (1977), and Ney (1977), and references therein, as well as parts of a book by Spitzer (1978).

Some of the properties of interstellar dust can be studied by comparing the spectra of selected nearby stars with those of more distant stars of the same types. For most cases, the wavelength-dependent extinction per unit column density is close to a standard function, shown in Fig. 12. This must be due to dust because the interstellar gas is very transparent at most nonionizing wavelengths. The extinction curve is almost proportional to $\lambda^{-1}$ for visual wavelengths. There is a strong broad feature near $\lambda \sim 2200 \AA$, and the re are also infrared features not shown in the figure. Different types of grains are important at different wavelengths. At some wavelengths, extinction is due to scattering and to absorption in roughly equal amounts, but the infrared and the $2200 \AA$ features are mostly absorption. Absorbed radiation usually heats a grain and is thermally reemitted at infrared wavelengths; some grains, made of silicates, metals, or graphite, can survive at temperatures up to $1000 \mathrm{~K}$ or more. Because grain temperatures are not necessarily related to gas temperatures, dust can exist in photoionized gas.

The galactic dust-to-gas ratio is said to be rather uniform, so that a hydrogen column density $N_{\mathrm{H}}$ gives an extinction optical depth at wavelength $\lambda=5000 \AA$,

$$
\tau_{d}(\lambda 5000) \approx\left(10^{-21.2} \mathrm{~cm}^{2}\right) N_{\mathrm{H}} .
$$

On the average, $n_{\mathrm{H}} \sim 1 \mathrm{~cm}^{-3}$ in our part of the galactic disk, with a half-thickness of about 300 light-years; this would imply $\tau_{d}(\lambda 5000) \geq 0.2$ toward extragalactic objects, except that the actual extinction is patchy because $n_{\mathrm{H}}$ is very nonuniform. Some astronomers have found practically no extinction in directions perpendicular to the galactic disk (e.g., Sandage, 1973; Feltz, 1972), but others have suggested minimum values of $\tau_{d}(\lambda 5000)$ between 0.04 and 0.2 , with 0.1 a likely compromise value (see Holmberg, 1974; Appenzeller, 1975; Knude, 1977; Burstein and Heiles, $1978 \mathrm{a}$, b, and references, therein). Values toward most quasars must be somewhat larger because their directions are not perpendicular to the galactic disk. Ultraviolet extinction is roughly 3 times as strong as at $5000 \AA$ (Fig. 12). Therefore we expect that extinction within our galaxy reduces most low-red-shift quasars' apparent $L \alpha / H \beta$ ratios by $10 \%$ to $60 \%$; perhaps $30 \%$ is typical. The effect is much less for a high-red-shift quasar, because differential extinction is smaller for the longer red-shifted wavelengths.

It is possible to imagine a larger effect, which is not excluded by presently available data. Suppose that there is a component of relatively small grains, which cause extinction mainly at ultraviolet wavelengths, extending to distances well beyond 300 light-years from the galactic plane. We would have no good way of knowing about such dust from pre-1978 observations, but there could be a significant effect on apparent ultraviolet/ visual intensity ratios for extragalactic objects.

\section{Intergalactic dust?}

We should not exclude the possibility that dust may be found anywhere along the line of sight to a quasar. Some absorption lines in quasars' spectra may be due

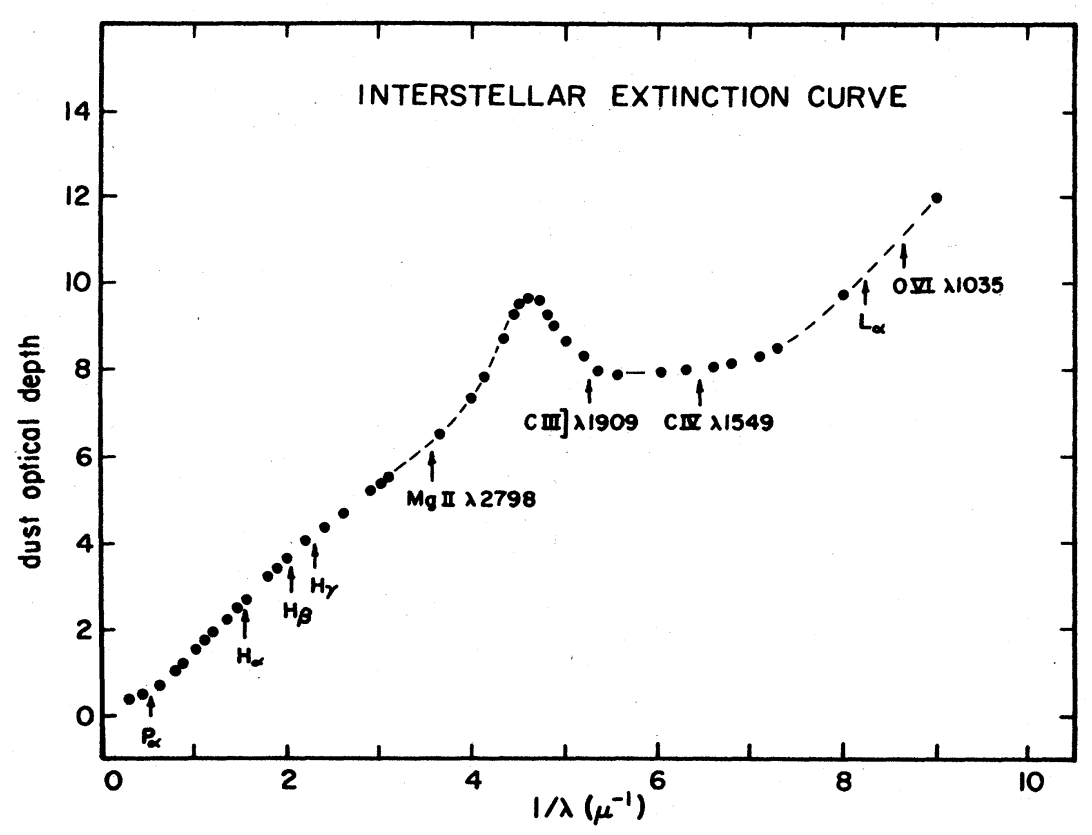

FIG. 12. Inte rstellar extinction in our Galaxy, as a function of wavelength. The data are taken from Schild (1977) and references therein. The extinction optical depth is normalized arbitrarily. 
to intervening galaxies or intergalactic gas clouds; dust grains may also be present there. The resulting extinction effects would tend to be greater for high-red-shift quasars, and this would oppose the red shift dependence of reddening in our Galaxy. The $2200 \AA$ feature, if present, would be smeared out by differential red shifts (as noted by Shuder and MacAlpine, 1979). There might also be tenuous dusty material filling clusters of galaxies around the quasars. See Schmidt (1976).

\section{Dust in quasars' galaxies}

Although the evidence is incomplete, we suppose that quasars occur in galaxies, probably in galactic nuclei. The analogy with Seyfert galaxies makes it plausible that spiral galaxies, containing dusty gas, are involved. ${ }^{10}$ The observed narrow forbidden lines may come from such gas, and a few quasars have observable emission-line "haloes" with large galaxy-sized radii (Kristian, 1973; Wampler et al., 1975; Stockton, 1976; Richstone and Oke, 1977; Green, Williams, and Morton, 1978; Bergeron, 1976). Interstellar dust may therefore be present at distances between 100 and $10^{5}$ light-years from a quasar. To cause extinction by a factor approaching 10 at ultraviolet wavelengths, with a dust-to-gas ratio like that in our Galaxy, the gas column density $N_{\mathrm{H}}$ would be of the order of $10^{21} \mathrm{~cm}^{-2}$ $\sim 10^{3} \mathrm{~cm}^{-3}$ light-years. This is not a terribly large value. Incidentally, the gas is likely to be ionized by the quasar; this is one reason why we should not expect the dust to be exactly like that in our Galaxy.

Some relevant points are: (i) Such extinction would occur in the same wavelength reference system as the emission spectrum. Resonance scattering can therefore increase the extinction of the central parts of the $L \alpha$ and CIV $\lambda 1549$ lines. (ii) Because most observations of quasars include galaxy-sized regions, scattering by dust is only indirectly important and the effective extinction is mostly due to absorption. (iii) The apparent continua probably show that the $2200 \AA$ absorption feature cannot be as strong as it is in our Galaxy, although this feature is perceptible in the spectrum of PHL 938 (Baldwin, 1977a; Drew, 1978).

We can roughly estimate the equilibrium grain temperatures that result from ultraviolet absorption and infrared reemission. For simplicity, suppose that the absorption-emission efficiency of a roughly spherical grain is proportional to $\lambda^{-1}$. Suppose that the quasar's radiation is equivalent in grain-heating effect to an ultraviolet luminosity $L\left(\sim 10^{45}-10^{48} \mathrm{erg} \mathrm{s}^{-1}\right)$ concentrated at a wavelength of $1000 \AA$. Then the equilibrium dust grain temperature at distance $r$ from the quasar is

$T_{d} \approx(170 \mathrm{~K})\left(\frac{L}{10^{46} \mathrm{erg} \mathrm{s}^{-1}}\right)^{0.2}\left(\frac{1000 \text { light-years }}{r}\right)^{0.4}$.

\footnotetext{
${ }^{10}$ Some radio sources with emission lines are at the centers of elliptical or giant elliptical galaxies, which are traditionally less dusty than spirals. If quasars are really in such galaxies, as sugge sted by Sandage (1972), Silk, Spinrad, and Field (1973), Hawkins (1978), Wehinger and Wyckoff (1978), and others, our discussion is weakened but not invalidated-the emission lines obviously show that some diffuse matter is present. See also Rowan-Robinson (1977).
}

The maximum temperatures at which dust can survive, $T_{d} \approx 1000-1500 \mathrm{~K}$, occur at $r \sim 1-100$ light-years, close to the locations of the dense emission-line regions.

Most of the grains' infrared radiation would be at rest wavelengths near $(30 \mu \mathrm{m})\left(T_{d} / 100 \mathrm{~K}\right)^{-1}$. (Note, however, that $T_{d}$ may span a considerable range in any one object.) Some quasars' spectra have "bumps" near $\lambda \sim 3 \mu \mathrm{m}$, and emission around $10 \mu \mathrm{m}$ may also be present, but the important wavelengths between $20 \mu \mathrm{m}$ and $100 \mu \mathrm{m}$ are practically unexplored (see Neugebauer, 1978; Neugebauer et al., 1979; Rieke, 1978). We must admit, however, that data on 3C 273 do not suggest the presence of a very large far-infrared flux (Hildebrand et al., 1977; Elias et al., 1978). Much of a quasar's intrinsic energy output may be in the soft-x-ray range (see below), where grains' absorption efficiencies are low; so we should not expect the total infrared flux to approach the total luminosity. If quasars vary over timescales of hundreds or thousands of years, their apparent infrared fluxes may be reduced by light-travel-time effects.

Polarization studies may be relevant. Dust grains cause polarization in two ways: polarization-deperdent extinction occurs if elongated grains are statistically aligned (see Chap. 8 of Spitzer, 1978), while polarization-dependent reflection is possible even if the grains are spherical. Nonthermal (e.g., synchrotron) processes can also produce polarized continua, but if the emission lines are polarized like the continuum, we may conclude that scattering and/or extinction have occurred. This appears to be the case for some Seyfert galaxies (Angel et al., 1976; Stockman, Angel, and Beaver, 1976), but most quasars are only weakly polarized, and at least parts of their polarizations are not due to dust grains (Stockman and Angel, 1978; Stockman, Angel, and Miley, 1979). Polarization, unfortunately, can only be a positive indicator of extinction or scattering; extinction can occur without polarization.

\section{E. Dust in quasars}

As implied above, some dust grains might exist, at temperatures of the order of $1000 \mathrm{~K}$, within a quasar's line-emitting regions. This possibility was acknowledged by Baldwin (1977a), Davidson (1977b), Davidsen, Hartig, and Fastie (1977), and Hyland, Becklin, and Neugebauer (1978), and has been considered in some detail by Baldwin and Netzer (1978), London (1979), Shuder and MacAlpine (1979), and Ferland and Netzer (1979).

Dust within a photoionized region can very effectively absorb the $L \alpha$ and other resonance lines. As explained in Sec. IV.A, resonance scattering increases the average escape path length by a factor $\bar{K}_{2} \sim 6$. This means that an ultraviolet resonance line can be strongly absorbed while nonresonance ultraviolet lines are only slightly affected and nonresonance visual-wavelength lines are practically unaffected. Since $\bar{K}_{2}$ is roughly the same for all resonance lines, the amount of destruction of such a line depends mainly upon the size of that line's creation zone. If the $L \alpha$ photon density is decreased by absorption, the population of the $n=2$ level of hydrogen is also decreased, and this would 
change the situation discussed in Sec. IV.C. Baldwin and Netzer (1978), Shuder and MacAlpine (1979), and Ferland and Netzer (1979) have concluded that such resonance-line absorption may be non-negligible in quasars but cannot account for factors exceeding 2 or 3 in ratios like $I(L \alpha) / I(H \beta)$. If $L \alpha$ and other resonance lines were more strongly reduced by absorption, then the apparent weaknesses of HeII $\lambda 1640$ and some other ultraviolet lines as well as the continuum, relative to $L \alpha$, would be difficult to understand. The suggested internal dustto-gas ratio, to give a moderate effect as discussed by the above-cited authors, is small-about $10 \%$ of the ratio in our Galaxy.

Internal dust can also absorb some of the ionizing radiation and thus modify the line generating functions. Unfortunately, it is difficult to know how the absorption efficiency varies with photon energy; one cannot safely extrapolate the curve shown in Fig. 12. A correlation between $\mathrm{He}^{+} / \mathrm{H}^{+}$ratios and infrared emission from dust, in some emission regions in our Galaxy, has been interpreted as an effect of differential extinction of $\mathrm{H}^{0}$-ionizing photons $(\varepsilon>13.6 \mathrm{eV}, \lambda<912 \AA)$ and $\mathrm{He}^{0}$ ionizing photons $(\varepsilon>24.6 \mathrm{eV}, \lambda<504 \AA)$; this has led to suggestions that $\tau_{d}$ continues to rise with photon energy at ionizing energies (Mezger, Smith, and Churchwell, 1974; Panagia and Smith, 1978). On the other hand, theoretical considerations suggest that dust grains' absorption efficiencies should not increase steeply at ionizing photon energies (Huffman, 1976, 1977; Jones et al., 1977). Very small grains (sizes $\lesssim 0.02 \mu \mathrm{m}$ ) cause increased extinction somewhat beyond $\varepsilon \sim 10 \mathrm{eV}$, but for $\varepsilon \gg 10 \mathrm{eV}$, the photon energies and frequencies must exceed characteristic solid-material energies and frequencies so much that grains become transparent except for internal photoionization. Baldwin and Netzer (1978) described photoionization calculations with dust such that $\tau_{d} \sim \lambda^{-1}$ and also $\tau_{d} \sim \lambda^{-2}$; Shuder and MacAlpine (1979) considered the case $\tau_{d} \sim$ const. (These are really just absorption efficiencies; scattering by dust was assumed to be mostly forward-directed and therefore unimportant for this problem). The former cases have the most drastic effects, cutting off the ionizing continua at high photon energies. Then, because the $\mathrm{H}^{+} / \mathrm{H}^{0}$ transition zone is less strongly heated by soft $\mathrm{x}$ rays, collisionally excited $L \alpha$ and $\mathrm{Mg} \Pi \lambda 2798$ are severely weakened. However, as noted above, real dust grains are probably not so effective for absorbing soft-x-ray photons.

Beyond the ionization front of a photoionized region, the $\mathrm{H}^{\circ}$ region may be extensive and may contain dust. In such a case, ultraviolet emission lines and perhaps even visual-wavelength lines can escape only through the illuminated (inner?) boundary of each cloud or filament. Ferland and Netzer (1979) tried to take this into account, but the uncertainties in dust properties (especially scattering details) are important.

\section{F. What is the intrinsic spectrum of a quasar?}

According to the preceding discussion, extinction by dust within a quasar's emission regions may be nonnegligible but the He II and O I lines suggest that "external" reddening is probably more important. This is most likely to occur in or near a galaxy surrounding
TABLE II. A possible "corrected" quasar's line spectrum. [Intensities relative to $I(L \alpha) \equiv 100$ ].

\begin{tabular}{lcccc}
\hline \hline Line & & $\begin{array}{c}\text { Apparent } \\
\text { intensity }\end{array}$ & $\begin{array}{c}\text { Corrected } \\
\text { for reddening }\end{array}$ & $\begin{array}{c}\text { Calculated } \\
\text { in model }\end{array}$ \\
\hline $\mathrm{O}_{\mathrm{VI}}$ & $\lambda 1034$ & 20 & 31 & 38 \\
$L \alpha$ & $\lambda 1215$ & 100 & 100 & $100^{\mathrm{a}}$ \\
$\mathrm{N} \mathrm{V}$ & $\lambda 1240$ & 25 & 24 & 27 \\
$\mathrm{O}_{\text {IV] }}$ & $\lambda 1402$ & 10 & 7 & 2 \\
$\left.\mathrm{~N}_{\text {IV }}\right]$ & $\lambda 1486$ & 3 & 2 & 3 \\
$\mathrm{C}_{\text {IV }}$ & $\lambda 1549$ & 40 & 23 & 30 \\
$\mathrm{He}$ II & $\lambda 1640$ & 5 & 2.6 & 3.5 \\
$\mathrm{O}_{\text {III] }}$ & $\lambda 1663$ & 3 & 1.5 & 1 \\
$\mathrm{C}_{\text {III] }}$ & $\lambda 1909$ & 18 & 7 & 5 \\
$\mathrm{Mg}_{\text {II }}$ & $\lambda 2798$ & 23 & 5 & 4 \\
$\mathrm{He}$ II & $\lambda 4686$ & 2.5 & 0.37 & 0.44 \\
$H \beta$ & $\lambda 4861$ & 17 & 2.5 & 1.7 \\
$\mathrm{He}$ I & $\lambda 5876$ & 4 & 0.5 & 0.1 \\
$H \alpha$ & $\lambda 6563$ & 77 & 9.6 & 7.7 \\
$P \alpha$ & $\lambda 18751$ & 6 & 0.5 & 0.4 \\
\hline \hline
\end{tabular}

${ }^{2} 22 \%$ of $L \alpha$ is due to recombination; $78 \%$ is due to collisional excitation of $\mathrm{H}^{0}$.

the quasar. If we make plausible corrections for reddening, the intrinsic continuum and relative line intensities are changed from those which were used in Sec. III; so photoionization analyses must be reconsidered. Here we shall describe just one suitably modified photoionization model.

Table II shows a typical, composite spectrum of line intensities, obtained by averaging many observed spectra. We have listed observed values as the "apparent" spectrum; then we have corrected these relative intensities for reddening, using an idealized extinction law $\tau_{d}(\lambda)=(3100 \AA / \lambda)$. This is very crude-no discrimination has been made between absorption and scattering by dust, nor has resonance scattering of $L \alpha$ been considered to affect the extinction-for our intent is merely to give a fair impression of how the situation is changed from Sec. III.

To be consistent, we should also correct the observed quasars' continuum shapes $F(\varepsilon)$ for reddening. If this is done using the idealized extinction law, then the resulting intrinsic visual-wavelength continua decrease only gradually toward the ultraviolet, while the corrected ultraviolet continua $(\varepsilon>5 \mathrm{eV}, \lambda<2500 \AA)$ actually increase toward higher photon energies; two different continuum emission processes may be indicated. But it is difficult to be more specific, because the true extinction law is probably more complicated than $\tau_{d} \sim \lambda^{-1}$ and this affects the curvature of the continuum on a $\log F(\varepsilon)-\log \varepsilon$ plot. Moreover, the observed continua are not all alike. We suspect that quasars' intrinsic continua in the $\varepsilon \sim 10 \mathrm{eV}$ region may resemble $F(\varepsilon)$ $\sim$ const or $F(\varepsilon) \sim \varepsilon^{0.5}$; even steeper spectra are possible. Most of the energy is presumably concentrated near a high-energy cutoff. A typical cutoff energy cannot be above $1 \mathrm{keV}$; otherwise, more quasars would be known sources in the several-keV x-ray region.

In the particular photoionization model described here (see Netzer and Davidson, 1979), the assumed continuum shape is $F(\varepsilon) \sim \varepsilon^{+1 / 3} e^{-\varepsilon / \varepsilon_{0}}$, with $\varepsilon_{0}=200 \mathrm{eV}, U_{\mathrm{H}}$ $=10^{8.2} \mathrm{~cm} \mathrm{~s}^{-1}$, and $n_{e} \sim 10^{9.6} \mathrm{~cm}^{-3}$. (The exponent $+1 / 3$ 
would match either thermal radiation from the most simplified, idealized sort of "accretion disk" or else a limiting case involving synchrotron radiation, but there is obviously no need to take this particular number very seriously. The specified value of $\varepsilon_{0}$ was chosen to give a satisfactory set of emission line intensities; if thermal, it would correspond to a maximum temperature just above $10^{6} \mathrm{~K}$.) The calculated plane-parallel, constant-gas-pressure photoionized region is optically thick to ionizing photons, with $\mathrm{C}, \mathrm{N}, \mathrm{O}$, and $\mathrm{Mg}$ about $80 \%$ as abundant as in the model described in Sec. III. B. The intense soft-x-ray flux around $\varepsilon \sim 200 \mathrm{eV}$ has the following effects: (i) The most highly ionized zones, near the illuminated boundary, are fairly hot $(T \sim 25000$ K) despite moderately large heavy-element abundances. OVI $\lambda 1034$ and N V $\lambda 1240$ emission are therefore so bright that a "second component" invoked in Sec. III is unnecessary. (ii) The $\mathrm{H}^{+} / \mathrm{H}^{0}$ transition zone is fairly hot $(T \sim 12000 \mathrm{~K})$, so that most hydrogen lines are strongly enhanced by collisional excitation. (iii) The total luminosity is much larger than the observable visual-ultraviolet continuum luminosity. This means that the observed emission line-to-continuum ratios can be explained if the line-emitting regions" "covering factors" are very small-i.e., even if less than $1 \%$ of the central luminosity is intercepted by photoionized regions. (iv) Although $U_{\mathrm{H}}$ is about the same as in Sec. III, the total pressure of ionizing radiation in each photoionized region is comparable to the gas pressure, rather than a factor of 10 smaller as in Sec. III.

There are only two nontrivial discrepancies in Table II. The calculated C IV] $\lambda 1402$ intensity is deficient by a factor of 3 , but this is only mildly objectionable: the relevant collision strength is uncertain, the ionization fraction of $\mathrm{O}^{3+}$ may be less certain than those of some other ion species, and Si IV emission may also be present. It is more disturbing that the calculated He I $\lambda 5876$ intensity is too small by a factor of 5 . However, this line is difficult to calculate reliably (see Sec. IV.D), and direct excitation of $\mathrm{He}^{0}$ bound levels by secondary electrons was not included here. Such fast electrons, freed in soft-X-ray photoionization events, are important in the low-ionization zones, causing ionization (which was included in the calculation) and excitation (which was not). So it is possible that the calculated He I $\lambda 5876$ intensity is indeed an underestimate.

Aside from these two discrepancies, the strong soft$x$-ray model is remarkably successful in accounting for the observed emission lines; O VI $\lambda 1034$ and $N V \lambda 1240$ in particular are more simply explained than in the traditional models discussed in Sec. III. The required elemental abundances and characteristic value of $U_{\mathrm{H}}$ are not very different from those in the earlier models. Models with dominant soft $\mathrm{x}$ rays were discussed, from different viewpoints, by Tarter and Salpeter (1969), Bergeron and Salpeter (1973), Bergeron and CollinSouffrin (1974), and Shields (1974). This type of model is very flexible, which may be considered either an advantage or a disadvantage. We can vary $U_{\mathrm{H}}, n_{e}$, and the elemental abundances (see Sec. III. C), as well as the power-law spectral index and cutoff energy of the ionizing continuum. (In particular, the total luminosity is reduced if the cutoff $\varepsilon_{0}$ is actually less than $200 \mathrm{eV}$-al- though such a model may need two components to account for the O VI and N V emission.) With special geometries and dust arrangements, and modified extinction curves, it is possible for the reddening to be different for continuum, nonresonance lines, and resonance lines. Many improved observations and arguments will be necessary before we can be confident about any particular model.

One can speculate that the hypothetical soft-x-ray flux may be thermal rather than nonthermal. Soft-xray thermal emission from shock-heated gas can occur in a central-accretion situation, or in collisions between gas clouds at relative speeds of several hundred $\mathrm{km} \mathrm{s}^{-1}$. The latter possibility has been mentioned or discussed by many authors, including Nussbaumer and Osterbrock (1970), Davidson (1972, 1973, 1977b), Daltabuit and Cox (1972), and especially Daltabuit, Cox, and MacAlpine (1978). The hypothetical thermal continuum (most prominent at extreme ultraviolet and soft$x$-ray wavelengths) may fluctuate less dramatically than the nonthermal emission which comes from a compact source. Correlations between red shift and apparentvisual-wavelength variability may be relevant to this point; see Uomote, Wills, and Wills (1976) and Wills (1976).

Arguments against the type of model outlined above are provided by one particular quasar, 3C 273. We have mentioned at the end of Sec. V. B that the apparent $P \alpha / H \beta$ intensity ratio for $3 \mathrm{C} 273$, if interpreted in the simplest way, suggests only slight reddening; we have noted in Sec. V.D that this object does not show a very large peak in its spectrum at infrared wavelengths; and in addition, the He II $\lambda 4686$ recombination line is very weak in $3 C 273$, which seems inconsistent with a model that includes many $\mathrm{He}^{+}$-ionizing photons. However, this quasar is generally thought to be atypical (partly because of the lack of He II $\lambda 4686$ ), and may be extreme in some ways. A very large value of $\tau_{P_{\alpha}}$ may explain the $P \alpha / H \beta$ ratio even if there is some reddening. If $U_{\mathrm{H}}$ is rather low, and the hydrogen lines are collisionally excited, the relative He II weakness may be accounted for; maybe the average electron density is unusually high. Much of the $2-20 \mu \mathrm{m}$ infrared radiation may indeed be emitted by dust grains, and for the sake of completeness, the $40-\mu \mathrm{m}$-wavelength region should be observed. Besides, if $3 \mathrm{C} 273$ fluctuates in luminosity with a timescale of 1000 years or so, and is now seen in an unusually bright state, then the dispersion in light-travel-times would reduce the observable infrared

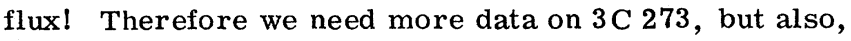
we need to understand how it is like and unlike other quasars. The same statement obviously applies to Seyfert galaxies.

\section{G. Comparing lines with continua}

As mentioned in Sec. III. A, one reason for the popularity of photoionization models is the fact that most quasars' spectra have roughly similar emission-lineto-continuum intensity ratios. Although such a result is not assured, a correlation between continuum brightness and ionized gas is suggestive of photoionization. The detailed interpretation of this correlation is affected if there is reddening by dust.

For clarity, imagine an oversimplified pure photo- 
ionization picture with no dust. Suppose that the hydrogen lines are entirely due to recombination; the continuum has the shape $F(\varepsilon) \sim \varepsilon^{-s}$; the absorption of each ionizing photon eventually leads to the emission of one recombination $L \alpha$ photon; and the $L \alpha / H \beta$ intensity ratio has a standard recombination value. Under these conditions, a measurement of either the $L \alpha$ or the $H \beta$ line, relative to the continuum, would clearly indicate the fraction of ionizing photons which are absorbed in photoionization events. Various complications must occur in practice-for example, part of each line may be due to collisional excitation of $\mathrm{H}^{\circ}$-but estimates of this type have been attempted for quasars by many authors, including Shklovsky (1965), Searle and Sargent (1968), Oke, Neugebauer, and Becklin (1970), Oke (1974), Baldwin $(1975,1977 \mathrm{a})$, Osmer and Smith (1976), Baldwin and Netzer (1978), and Neugebauer et al. (1979). The Balmer lines in low-red-shift spectra have seemed to imply that most of the ionizing continuum is usually absorbed, but the $L \alpha$ emission in high-red-shift spectra has appeared to require only a fraction $(\sim 10 \%$ ? ) of the ionizing photons; this discrepancy is of course a manifestation of the " $L \alpha / H \beta$ problem."

The photoionized regions are probably optically thick to most ionizing photons (see arguments cited in Sec. III; Davidson, 1976; and other aspects mentioned below). Therefore the fraction of ionizing photons which are absorbed can be interpreted as a "covering factor," i.e., the fraction of sky which is covered by the photoionized regions as seen from a hypothetical central source. (We assume that the ionizing radiation is not "beamed" in particular directions.) Another way to guess the covering factor is to observe the continuum near the Lyman edge (the $\mathrm{H}^{0}$ ionization threshold: rest wavelength $912 \AA$ ). If, for example, the average quasar's covering factor is 0.2 , then one might naively expect $20 \%$ of the quasars' spectra to show absorption discontinuities near the Lyman edge, due to the Lyman continuum and perhaps the highest-order Lyman lines [see Bahcall and Sargent (1967) for an early view of this idea]. In fact, some high-red-shift quasars have no Lyman discontinuities in their spectra, while others have discontinuities at absorption-system red shifts which are probably unrelated to the dense line-emitting regions; but discontinuities of the naively expected type are quite rare (see Oke, 1974; Beaver et al., 1976; Baldw in et al., 1976; Osmer and Smith, 1977b; Osmer, 1978; Wright et al., 1978). The average geometrical covering factor seems to be less than 0.2. Independent evidence that covering factors are less than unity, at least in a few cases, is provided by the fact that some quasars are compact radio sources. Dense ionized regions of the types discussed in Sec. III are quite opaque at wavelengths much greater than $1 \mathrm{~mm}$. These covering-factor arguments may be taken as evidence against photoionization models in which resonance lines are suppressed by internal dust absorption or by collisional deexcitation. If, for instance, the apparent $L \alpha$ intensity has been reduced by such effects, but the apparent continuum has not, then a large covering factor is required-which may be inconsistent with the facts that we have just mentioned.

It may be that a quasar whose continuum source is obscured by a dense emission-line region would not be recognizable. Imagine, for example, that the dense gas is dusty and arranged in a disk; such a configuration might be inconspicuous when viewed edge-on. The idea of a disklike or toroidal arrangement of gas has been discussed by various authors, including Shields (1977) and Osterbrock (1978c). Incidentally, in this discussion we do not consider certain peculiar objects like PHL 5200, whose spectra have broad absorption lines on the short-wavelength sides of ultraviolet emission lines. Such objects are probably surrounded by envelopes of ejected gas (which need not be dense), and the "emission lines" may really be due to scattering - their intensities resemble the intensities which have been removed from the continuum in the adjoining absorption features. [See Lynds (1967), Scargle, Caroff, and Noerdlinger (1970), Hewitt and Noerdlinger (1974), Marti and Noerdlinger (1977), Weedman et al. (1979), and refrences cited by Perry, Burbidge, and Burbidge (1978).]

If covering factors are small, why do the Balmer lines seem to indicate covering factors near unity? Two obvious answers are (1) the Balmer lines may be enhanced by collisional excitation, and/or (2) the naive power-law extrapolations of visual continua may be invalid. Both of these explanations are correct in a model like that outlined in Sec. V. F. Moreover, because so much far-UV and soft-x-ray luminosity is available in such a model, the covering factor can be far less than 0.1 .

What happens to most of the ionizing photons? If there is extinction by dust, outside the dense emissionline regions but still within the quasar's galaxy, then gas must also be present along with the dust; perhaps this gas can be identified with the narrow-line-emitting regions. But since we do not observe strong emission lines from such gas, or strong Lyman-continuum absorption, it must not absorb most of the ionizing photons. Without going into details, we note that this places a lower limit on $U_{\mathrm{H}}$ in the hypothetical extinction-causing material: if $U_{\mathrm{H}} \gtrsim 10^{9} \mathrm{~cm} \mathrm{~s}^{-1}$, most of the ionizing photons can be absorbed by the dust rather than by the gas. For several quasars, there is evidence that only small fractions of their ionizing luminosities are being absorbed in photoionization events within $10^{6}$ light-years of the sources (Davidson, 1977a, 1978; Sargent and Boroson, 1979) - although we do not wish to insist here that a quasar's luminosity remains steady as long as $10^{6}$ years! See also Arons and Wingert (1972) and Osmer (1978). (Strong continuum fluctuations in about 1000 years, as mentioned above in connection with the infrared, might explain the absence of strong forbidden lines in another way-involving light-traveltimes. This explanation would require quasars to be "turned off" most of the time.)

A related problem concerns correlations between luminosities and emission-line-to-continuum ratios. Baldwin (1977b) suggested that quasars' $L \alpha$ and/or C IV $\lambda 1549$ intensities, measured relative to the underlying continua, tend to be smaller for more luminous quasars; this statement appears to have been confirmed for the C IV line, but is not really settled yet (Osmer, 1977; Baldwin et al., 1978; Smith, 1978). It is easy to invent speculative explanations of such correlations (whether or not the correlations are real!), but it is 
difficult to decide which explanations are correct. The suspected correlations cannot continue down to the lower luminosities of Seyfert galaxies, if photoionization models are valid, because then the line-to-continuum ratios would require covering factors larger than unity.

The idea of significant extinction by dust, which has been favored in this section, may require quasars to produce embarrassingly monstrous luminosities. Many of them appear to have ultraviolet luminosities $\varepsilon L_{\varepsilon}$ $=\lambda L_{\lambda} \sim 10^{47} \mathrm{erg} \mathrm{s}^{-1}$, and a few extraordinary objects sometimes approach $10^{48} \mathrm{erg} \mathrm{s}^{-1}$ (Eachus and Liller, 1975; Ulrich, 1976; Osmer and Smith, 1977a,b; Wright and Kleinmann, 1978; Gottlieb and Liller, 1978). The proposed corrections for extinction would raise these ultraviolet values to several times $10^{48} \mathrm{erg} \mathrm{s}^{-1}$, and may lead to soft-x-ray luminosities of the order of $10^{50}$ erg $\mathrm{s}^{-1}$ ! By adjusting our models (e.g., by moving the intrinsic peak to $\varepsilon \sim 20 \mathrm{eV}$ rather than $200 \mathrm{eV}$ ), we can probably reduce the maximum luminosity to about $10^{49}$ $\mathrm{erg} \mathrm{s}^{-1}$, but in any case these values are too large for comfort. The required compact objects would probably have the masses of galaxies, with "fuel" consumption rates up to 1000 solar masses per year. The overall sizes of the emission-line regions would be larger than those suggested in Sec. III. B. Nevertheless, we do not think that these numbers are quite large enough to amount to a proof of impossibility.

\section{SOME ADDITIONAL SPECTRAL FEATURES}

\section{A. Forbidden lines}

We shall not discuss quasars' narrow forbidden lines in much detail, for several reasons: they are not associated with the mysterious centers of activity as closely as the broad lines are; they do not carry as much energy as the permitted lines; they are very density dependent; and most of their implications can be derived by the traditional methods of nebular analysis which have been extensively reviewed in the astronomical literature. We have already cited some of these reviews in Sec. II. A; for applications to Seyfert galaxies and quasars, see Osterbrock (1978b) and references therein. Some relevant photoionization calculations have been described by Williams and Weymann (1968), Tucker and Tarter (1968), Yankulova (1974), Shields (1974), Shields and Oke (1975), and Boksenberg and Netzer (1977).

Quasars' forbidden lines must originate in relatively low-density gas $\left(n_{e}<10^{7} \mathrm{~cm}^{-3}\right)$ and are narrower than the permitted lines (widths correspond to several hundred $\mathrm{km} \mathrm{s}^{-1}$ vs several thousand $\mathrm{km} \mathrm{s}^{-1}$ ). In some spectra, narrow Balmer lines, evidently produced in the forbidden-line gas, can be seen superimposed on the broad Balmer lines. The forbidden lines represent a wide variety of stages of ionization, including $\left[\mathrm{O}_{\mathrm{I}}\right]$, [S II $],[\mathrm{N} \mathrm{II}],[\mathrm{O} \mathrm{II}],[\mathrm{O} \mathrm{III}],[\mathrm{Ne} \mathrm{III}]$, $[\mathrm{Ne} \mathrm{V}],[\mathrm{FeVII}],[\mathrm{Fe} \mathrm{X}]$, and $[\mathrm{Fe} \mathrm{XI}]$. As one might expect, the forbidden-line regions are not very close to the centers of activity. In some nearby Seyfert galaxies (e.g., NGC 7469; see Ulrich, 1972), the forbiddenline zones can be partly resolved and are several hundred light-years across; these dimensions should probably be scaled up for more luminous quasars. Some quasars are known to have galaxy -sized forbidden-line "haloes" (Wampler et al., 1975; Stockton, 1976; Richstone and Oke, 1977; Bergeron, 1976). Furthermore, some Seyfert galaxies' forbidden lines remain constant while their broad lines vary; this probably means that the forbidden-line regions are much larger than the broad-line regions. Dynamical arguments, involving the velocity dispersions implied by the linewidths, lead to similar conclusions. Like the denser broad-line gas, the forbidden-line regions probably do not fill the available volumes and may consist of many clouds, filaments, or sheets.

The forbidden lines are not as bright as the broad lines, either because the narrow-line regions have a smaller overall covering factor than the broad-line regions, or because the forbidden-line gas is optically thin to ionizing radiation, or because of dust, or because of fairly long-timescale continuum variability. Nevertheless, considering the density dependences of emissivities, the amount of gas represented by forbidden lines must greatly exceed that which produces the broad lines. A quasar's dense regions, discussed in Secs. III. and IV, may contain only 100 or 1000 solar masses of diffuse photoionized material (of course, there may also be some related nonionized gas); the forbidden-line regions may be $10^{2}$ to $10^{6}$ times more massive. The intensity ratios of certain forbidden lines, particularly [O III ] $\lambda 5007 / \lambda 4363$ and [S II] $\lambda 6731 / \lambda 6717$, give information about temperatures and densities (see references cited above); $T \sim 10^{4}$ to $3 \times 10^{4} \mathrm{~K}, n_{e} \sim 10^{3}$ to $10^{6} \mathrm{~cm}^{-3}$ are typical guesses, which allow photoionization as a possible dominant heating mechanism. Heating by shock fronts may also be important. Photoionization models, adjusted to produce the high-ionization forbidden lines, generally do not produce adequate [O II] and [S II] emission. Some possible ways of enhancing the low-excitation lines through geometrical or time-dependence assumptions have been described by Shields and Oke (1975); but it should be noted that low-ionization line intensities are difficult to explain even in ordinary nebulae in our Galaxy. Phillips (1978a) has remarked that high-ionization forbidden lines ( [O III] in particular) are in some cases slightly blue-shifted relative to lower-ionization forbidden lines. This presumably indicates that the lines preferentially originate in different zones. (If the relative shift is always in the same sense, this also leads us to suspect that a typical configuration is symmetric, with some dust to obscure emission in particular places and/or directions.) It has been generally difficult or impossible to deduce elemental abundances from quasars' and Seyferts' forbidden lines.

If the $\mathrm{Fe}^{9 .+}$ and $\mathrm{Fe}^{10+}$ ions (required to give observed [Fe $\mathrm{X}]$ and [Fe XI] lines) are photoionized, then there must be significant soft-x-ray fluxes at photon energies near $250 \mathrm{eV}$. Alternatively, as Oke and Sargent (1968) suggested, there may be enough hot $\left(T \sim 10^{6} \mathrm{~K}\right)$, lowdensity $\left(n_{e} \sim 100 \mathrm{~cm}^{-3}\right)$, collisionally ionized gas to produce such lines. It is interesting that the [Fe XI] $\lambda 7892$ line is broader than the other forbidden lines in some spectra (Grandi, 1978). The critical density for collisional deexcitation of this line is of the order of $10^{9}$ $\mathrm{cm}^{-3}$, so it may originate in at least the lower-density 
parts of the broad-line regions.

Incidentally, one should be skeptical of any photoionization calculations of iron lines. There are too many stages of ionization of iron, each very poorly known regarding ionization and excitation cross sections, recombination rates, charge-exchange reactions, etc., for this element to be treated decently.

\section{B. Fe II lines}

Wampler and Oke (1967) identified some broad emis sion features in the spectrum of the quasar $3 \mathrm{C} 273$ as blends of several permitted Fe II multiplets. The strongest blends are around $\lambda \sim 4570,5190$, and $5320 \AA$. Sargent (1968) identified similar features in the spectrum of a Seyfert galaxy, and Fe II emission has since been observed in many quasars' and Seyferts' spectra (see Fig. 3). Extensive observational work on this subject has been reported by Osterbrock (1977) and Phillips (1978a); it appears that perceptible Fe II emission is produced by most "Type 1" Seyfert nuclei but is less common in quasars. The lines are broad and therefore probably originate in the dense central regions. In some cases, the total of the Fe II intensities exceeds the $H \beta$ intensity.

Some nebulae and peculiar stars in our Galaxy have permitted FeII as well as forbidden [FeII] emission in their spectra (e.g., see Thackeray, 1967; Viotti, 1976). We generally do not understand such features in quantitative detail, because $\mathrm{Fe}^{+}$is such a complicated ion that many of its relevant parameters are only vaguely known. The near-ground levels of $\mathrm{Fe}^{+}$have even parity; we shall call them "levels 1." About $3 \mathrm{eV}$ above them are some more even-parity levels 2 , and about $5 \mathrm{eV}$ above the ground level are some odd -parity levels 3 . There are many permitted transitions $1-3\left(\lambda \sim 2300_{-}^{-}\right.$ $2800 \AA$ ) and $2-3$ (mostly $4000-6000 \AA)$, and forbidden transitions 1-2 (mostly 3500-5500 A). The lines observed in quasārs' and Seyferts' spectra correspond to the 2-3 transitions; $1-2$ and $1-3$ transitions are not represented. The absence of the forbidden 1-2 lines probably indicates high densities, $n_{e}>10^{7} \mathrm{~cm}^{-3}$. However, the absence of permitted $1-3$ lines is not quite as easy to explain, considering that they have larger transition rates than the observed lines. Presumably, resonance scattering plays a role here; we can also imagine observational complications involving reddening, the "3000 ̊ bump" (see Sec. VI. C), and perhaps even the $2200 \AA$ dust feature. It should be noted that $\mathrm{Fe}^{+}$, like $\mathrm{Mg}^{+}$, is easily ionized and therefore should be scarce in a photoionized $\mathrm{H}^{+}$region (unless $U_{\mathrm{H}}$ is very low). Most of the $\mathrm{Fe}^{*}$ ions should be found in the $\mathrm{H}^{+} / \mathrm{H}^{\circ}$ transition zones and in $\mathrm{H}^{\circ}$ regions.

What is the excitation mechanism? Ordinary recombination is almost certainly inadequate; the observed Fe II intensities would require such a large iron abundance that other iron-ion features would appear, although some sort of charge-exchange reaction might conceivably have the same effect as very efficient recombination. The most likely mechanisms are collisional excitation, and fluorescence in which continuum photons are absorbed in the 1-3 quasiresonance lines. Phillips (1978b) and Collin-Souffrin et al. (1979) have discussed these processes.
By comparison with $\mathrm{Mg}$ II $\lambda 2798$, Phillips stated that an implausibly large $\mathrm{Fe}^{+} / \mathrm{Mg}^{+}$abundance ratio would be required to produce the observed intensities by collisional excitation. Also, with collisional excitation, the observed relative intensities of various Fe II features are difficult to explain and the ultraviolet $1-\underline{3}$ lines should appear in emission. Nevertheless, this mechanism should not yet be excluded as a possibility (see Boksenberg and Netzer, 1977). Collin-Souffrin et al. (1979) have proposed collisional excitation in a rather hot, nonphotoionized gas.

FeII fluorescence was proposed by Wampler and Oke (1967) and further discussed by Bahcall and Kozlovsky (1969a), Osterbrock (1976), Oke and Shields (1976), and others. The idea is that some of a quasar's continuum photons can be absorbed in Fe II 1-3 quasiresonance lines. Most of the resulting level $\overline{3} \overline{\mathrm{F}} \mathrm{e}^{+}$ions will decay directly to levels $\underline{1}$, but if resonance scattering operates in a favorable manner, the indirect decay routes $3-2-1$ may be important. The desired emission results from $3-2$ decays while the 2-1 transitions are mostly collisional rather than radiative if $n_{e}>10^{7} \mathrm{~cm}^{-3}$. In order to get the observed intensities, each 1-3 line must remove part of the total continuum equivalent to at least $\Delta \lambda>1 \AA$ or $\Delta v$ (Doppler broadening) $>100 \mathrm{~km} \mathrm{~s}^{-1}$.

Adams (1975a) described some 3-level simplified models for this process, and Phillips (1978b) and Collin-Souffrin et al. (1979) have constructed more elaborate multilevel models. The FeII-emission zones in these models are essentially uniform in temperature and density, although the regions of interest are probably $\mathrm{H}^{+} / \mathrm{H}^{0}$ transition zones. Collin-Souffrin et al. remarked that $\mathrm{Fe}^{++}$should be more common than $\mathrm{Fe}^{+}$, even in a $\mathrm{H}^{+} / \mathrm{H}^{0}$ transition zone, because of photoionization from excited levels; but perhaps charge exchange is important.

The picture which seems most favorable for Fe II fluorescence, while being consistent with most of our discussion in preceding sections, has the following features: (i) Local velocity dispersions (within individual clouds, filaments, or sheets) are small, of the order of $10 \mathrm{~km} \mathrm{~s}^{-1}$, and each local region is optically thick in each Fe II 1-3 line. (ii) There are larger-scale velocity dispersions considerably greater than $100 \mathrm{~km} \mathrm{~s}^{-1}$ in some radial directions, so that enough continuum radiation can be absorbed. Phillips mentions hydrogen column densities $N_{\mathrm{H}} \sim 10^{21}$ to $10^{23} \mathrm{~cm}^{-2}$. (iii) The overall velocity dispersion is several thousand $\mathrm{km} \mathrm{s}^{-1}$, in order to give the observed overall linewidths. (iv) Covering factors are much less than unity (at least in the quasars, if not the Seyferts), so that we do not observe the Fe II 1-3 lines in absorption. Such a model may just barely be able to account for the observed Fe II intensities.

According to Phillips (1978b), a further difficulty with fluorescence as the dominant mechanism for Fe II emission is the absence of some Si II lines that should be produced in the same way. At this time, it is difficult to wholeheartedly accept or exclude either fluorescence or collisional excitation of the Fe II emission.

Surprisingly, there are no definite, confirmed correlations between observed Fe II emission and other spectral features; but at least two possibilities have been 
suggested. (i) Fe II emission may be anticorrelated with radio emission (Osterbrock, 1977; Grandi and Osterbrock, 1978); and (ii) Allen (1976) has proposed a correlation between Fe II emission and infrared emission in Seyfert galaxies, more or less by analogy with a similar correlation among objects in our Galaxy. The latter suggestion, if correct, may imply that Seyferts are dustier than quasars.

In the spectrum of the Seyfert galaxy NGC 4151, there are forbidden [Fe II] lines, originating in the less dense narrow-line regions (Boksenberg et al., 1975; Netzer, 1974). This interesting object is peculiar in several ways, which we shall not discuss here.

\section{The "3000 A bump"}

Many quasars' and Seyfert galaxies' spectra have broad "bumps" in the wavelength range 3000-4000 $\AA$; see Oke, Neugebauer, and Becklin (1970), Shields, Oke, and Sargent (1972), Baldwin (1975), Phillips (1978a), and Neugebauer et al. (1979). In some cases this may be the Balmer continuum in emission $(\lambda \lesssim 3645 \AA)$, but in other cases the intensity is too large and the peak wavelength is too small. Among additional suggested contributors to the feature are Fe II emission, and "two-photon" emission produced by $2 s-1 s$ decays of hydrogen. The latter seems implausible because collisional $2 s-2 p$ transitions should be faster than two-photon decays at reasonable densities $n_{e} \gg 10^{4} \mathrm{~cm}^{-3}$; but it may occur in $\mathrm{H}^{0-}$ zones, where decay from $2 p$ is in effect suppressed by enormous $L \alpha$ optical depths.

This somewhat mysterious spectral feature may be relevant to the possibility of extinction by dust grains (Sec. V above). At visual rest wavelengths $(\lambda>4000 \AA)$, extinction is expected to be small or moderate, while the ultraviolet $(\lambda<2000 \AA)$ extinction is uncertain but may be large. Because intermediate wavelengths include the Balmer continuum, possible extra emission, and a possible $2200 \AA$ extinction feature, it is difficult to join the visual and ultraviolet continua smoothly. The $3000 \AA$ bump may disguise the presence of a $2200 \AA$ feature or may even fill it in to some extent. One can even speculate that the $3000 \AA$ feature is partly due to dust. Energy deposited in a dust grain is normally assumed to be quickly thermalized and then reemitted at infrared wavelengths, but perhaps this is not always correct. Perhaps some effects (photoionization and recombination? disruption by fast charged particles? perhaps secondary electrons?) produce more direct radiation-scintillation-from electronic transitions in grains. The photon energies around $3000 \AA$ seem not unreasonable, and transitions in a grain produce diffuse spectral features rather than sharp lines.

\section{SOME ADDITIONAL POSSIBILITIES}

\section{A. Concerning the absence of broad forbidden lines}

Forbidden lines are not very intense in a typical quasar's spectrum, and broad forbidden lines are very faint. Therefore most authors suppose that the broadline emission regions are so dense $\left(n_{e}>10^{7}\right.$ or $\left.10^{8} \mathrm{~cm}^{-3}\right)$ that forbidden lines are suppressed by collisional deexcitation. But this has occasionally been doubted. Two obvious alternative ways to explain reduced inten- sity ratios such as $I([\mathrm{O}$ III $] \lambda 5007) / I(\mathrm{C}$ IV $\lambda 1549)$, as mentioned by Davidson (1972), are (i) the carbon/oxy gen abundance ratio may be large, and/or (ii) the temperature may be high so that the lower excitation energy of $\lambda 5007$ becomes less advantageous, relative to 71549. Possibility (i) is unlikely in view of the prominence of $O$ VI $\lambda 1034$, and possibility (ii) appears inconsistent with the absence of C III $\lambda 977$ (see Sec. III. A), not to mention other implausibility factors. Scargle, Caroff, and Tarter (1974) constructed a low-density model whose forbidden lines were not very prominent; but they did not clearly distinguish between broad and narrow lines, and their calculated forbidden-line intensities were larger than in observed broad-line spectra.

Some interesting mechanisms, particularly relevant to [O III] emission, were proposed by Burbidge et al. (1966), Burgess (1967), and Pfleiderer and Grewing (1967), and have been rediscussed by Shull and McGray (1978). Essentially, the idea is that radiation densities may be so large and forbidden transition rates so slow that an excited $\mathrm{O}^{++}$ion is usually photoionized or photoexcited before it creates a forbidden-line photon. If this happens, the forbidden lines may be weak even at low gas densities; and the relative intensities of forbidden lines, e.g., [O III] $\lambda 4363 / \lambda 5007$, may be altered. However, for these processes to be more effective than collisional deexcitation in suppressing [O III] $\lambda 5007$ emission, the radiation-to-gas ratio $U_{\mathrm{H}}$ must be very large, $U_{\mathrm{H}}>10^{11} \mathrm{~cm} \mathrm{~s}^{-1}$. Such values of $U_{\mathrm{H}}$ would entail radiation pressures which greatly exceed gas pressures, and would be very difficult to reconcile with existing photoionization results.

Although the radiative processes cited above do not invalidate the usual lower limits on gas densities, they do affect the $\mathrm{O}^{3 *} / \mathrm{O}^{++}$ionization ratios in dense regions and should be included in photoionization calculations. As explained in Sec. II. A, because the ionization threshold of $\mathrm{He}^{+}$is $54.4 \mathrm{eV}$, a typical photoionized region has a zone of $\mathrm{H}^{+}$and $\mathrm{He}^{+}$where the local ionizing continuum has a cutoff at $\varepsilon=54.4 \mathrm{eV}$ (although there may be some penetrating photons above $100 \mathrm{eV}$ ). This is the zone where $\mathrm{O}^{++}$traditionally occurs, because $54.9 \mathrm{eV}$ is required to ionize ground-level $\mathrm{O}^{++}$. But only $52.4 \mathrm{eV}$ is required to ionize $\mathrm{O}^{+*}$ from its lowest metastable (forbidden-line) level; and in a quasar-related model, precisely because densities are high, almost $10 \%$ of the $\mathrm{O}^{++}$ions are in this excited level. Therefore it is possible for continuum photons between 52.4 and 54.4 $\mathrm{eV}$ to change some of the $\mathrm{O}^{++}$to $\mathrm{O}^{3+}$. This effect is quite important for conventional power-law spectra with $U_{\mathrm{H}}$ $z 10^{8.5} \mathrm{~cm} \mathrm{~s}^{-1}$ and can affect the intensities of $\mathrm{O}$ III $]$ $\lambda 1663$ and O IV] $\lambda 1402$ (cf. Appendix B of Baldwin and Netzer, 1979). Davidson (1972) hoped for the sake of simplicity that absorption of high-order He II "Lyman series" lines would eliminate this effect, but the required local linewidths are probably excessive.

An argument against low densities, independent of forbidden lines, is provided if broad-line intensities are seen to vary with time scales of a few years. If suspected values of $U_{\mathrm{H}}$ are realistic, then dense emission-line gas must be distributed over regions several light-years across, or less-dense gas must be distri- 
buted over much larger regions. Considering travel times for light, we are not very surprised if dense-region line intensities vary in a few years, but no line variations should appear if densities are low.

\section{B. Can densities be surprisingly high?}

We think that the observed semiforbidden lines, especially C III $\rfloor \lambda 1909$, show that typical electron densities in the broad-line regions do not greatly exceed $10^{10}$ $\mathrm{cm}^{-3}$. However, we cannot absolutely prove this upper limit, and the " $L \alpha / H \beta$ problem" has led to suspicions that higher densities may be important. No self-consistent photoionization models (or, indeed, self-consistent alternative models) are available for such high densities, because serious complications occur for $n_{e}$ $z 10^{11} \mathrm{~cm}^{-3}$ : permitted transitions become collisionally deexcited in a radiative-transfer-dependent manner, highly excited levels and also the bremsstrahlung continuum become more important as resonance lines are saturated, three-body recombination (e.g., $2 e^{-}+\mathrm{H}^{+}$ $\left.\rightarrow \mathrm{H}^{0}+e^{-}\right)$becomes important, etc. We can imagine some likely difficulties in a very high-density model: (i) Somehow, the observed C III $\lambda 1909 / L \alpha$ intensity ratio must be obtained even though $\lambda 1909$ is far more severely reduced by collisional deexcitation than $L \alpha$ is, and this must be done without introducing much $\mathrm{C}$ III $\lambda 977$ emission. If strong nonphotoionization heating is invoked, the resulting high temperatures may violate the $\lambda 977$ limit. The only obvious way of getting adequate C III ] emission is to suppose that carbon is surprisingly abundant, $n_{\mathrm{C}} / n_{\mathrm{H}} \gtrless 10^{-2.5}$. Other semiforbidden lines, including $\mathrm{N}$ III ] $\lambda 1750$ and $\mathrm{N} \mathrm{IV}] \lambda 1486$, must also appear. (ii) The hydrogen lines must have satisfactory ratios. Extreme "thermalization", as proposed by Zirin (1978), might result in $L \beta / L \alpha$ and $H \beta / H \alpha$ ratios which do not resemble the observations. (iii) The He I lines must have satisfactory ratios even though they appear to indicate lower electron densities (see Sec. IV. D). (iv) Aside from fluorescence, there must be no perceptible visual-wavelength emission lines from highly excited levels of carbon, nitrogen, and oxygen ions. Such lines appear in the spectra of some stars with hot gaseous envelopes (see Underhill, 1966). (v) The partial suppression of major emission lines must not cause bremsstrahlung and radiative recombination to assume too much of the cooling burden, because then the total energy-supply problem would become difficult. These points, taken together, show why we are unenthusiastic about the very-high-density possibility. From astronomical experience, we know that the spectra of ordinary low-density nebulae are recognizably different from the spectra of higher-density hot stellar envelopes; and except for the easily understandable lack of forbidden lines, quasars' line spectra look nebular and not stellar.

\section{Additional heating processes}

Because so many far-ultraviolet continuum photons are obviously present, photoionization is virtually certain to be important in a quasar's line-emitting regions; but some additional heating may result from other processes. Purely photoionized regions are remarkably cool for their levels of ionization, so these other processes will probably affect the heating more than they affect the ionization. There are two likely effects: (i) photoionized regions, basically like those discussed in preceding sections, may be slightly hotter than in the standard calculations, and/or (ii) there may be adjoining regions of lower density, which are very hot because their heating is dominated by processes other than photoionization. Low-density, high-temperature gas is likely to be very transparent and can seriously affect, at most, only the high-ionization emission lines; but it may be dynamically important-it can restrain or confine the photoionized regions if instabilities are not too serious.

Energetic, nonthermal charged particles can heat gas by causing excitations and ionizations, and by Coulomb scattering; such heating has been discussed by Osterbrock and Parker (1965), Eilik and Caroff (1976), and others. The least energetic particles are most effective for heating purposes. Protons or electrons with energies below $1 \mathrm{MeV}$ lose energy so quickly that they cannot penetrate far into "thick" photoionized regions with $U_{\mathrm{H}} \gtrless 10^{8}$; such particles, if present in large numbers, may create thin, extremely hot edges or "coronae" around our hypothetical photoionized clouds or filaments. Charged particles far above 10 or $100 \mathrm{MeV}$ do not lose energy to the gas very quickly, so a very large energy density of such particles-implying a pressure greater than that of the gas-would be required to cause significant extra heating of the photoionized regions. Regarding particles of several $\mathrm{MeV}$, we note that a magnetic field of only $10^{-5} \mathrm{G}$ would make their Larmor orbits much smaller than the thicknesses of the photoionized zones; and actual ambient fields are probably much stronger, because the energy density associated with $10^{-5} \mathrm{G}$ is 10 orders of magnitude smaller than the likely thermal energy densities in the lineemitting gas. Therefore effective charged particles can enter the photoionized zones only along magnetic field lines. The magnetic field configuration (which we do not know) must affect the results of any calculation; so we feel uneasy about any calculated model of heating by charged particles. Since large energy densities of several $\mathrm{MeV}$ particles would be necessary to obtain strong effects, and considering that there is no evident reason why any hypothetical particle fluxes should be concentrated in this energy range, we suspect that this form of heating is unimportant in a quasar's line-emitting gas.

Next, there is compressive heating by shock waves. Direct heating of the line-emitting regions by shocks is unattractive. To obtain significant extra heating in photoionized gas, but not so much heating that the emission spectrum becomes unsatisfactory (i.e., to obtain localized temperature enhancements between 1000 and $30000 \mathrm{~K}$ ), the speed of a shock front must be of the order of $v_{\text {shock }} \sim 30 \mathrm{~km} \mathrm{~s}^{-1}$, which is rather low in the sense that each shock carries only a miniscule amount of energy by quasarian standards. To produce a significant average luminosity effect $\Delta L \sim 10^{44} \mathrm{erg} \mathrm{s}^{-1}$, the shocks would have to sweep through material at a rate of the order of $\Delta L / v_{\text {shock }}^{2} \sim 10^{31} \mathrm{~g} \mathrm{~s}^{-1} \sim 10^{5}$ solar masses per year; each cloud or filament would have to experience repeated shocks with recurrence times not much great- 
er than the characteristic gas cooling time, which is less than an hour for $n_{e} \sim 10^{9}$ to $10^{10} \mathrm{~cm}^{-3}$. It is not obvious how this could be made to work.

Shock waves are more interesting in a different way, as sources of ionizing radiation. Nussbaumer and Osterbrock (1970) mentioned that ionizing radiation is emitted in collisions between fast-moving gas clouds, and Davidson (1972) proposed the compressed parts of colliding clouds as suitable locales for a quasar's dense line-emitting regions. Daltabuit and Cox (1972), combining these aspects, proposed that colliding clouds contain both the sources of ionizing radiation and the photoionized regions. Consider an idealized picture, in which two identical clouds collide head-on at relative speed $V$ which may be of the order of $1000 \mathrm{~km} \mathrm{~s}^{-1}$. The ions and electrons of the two separate clouds cannot interpenetrate because there are small magnetic fields imbedded within the clouds. Therefore, as the collision proceeds, a shock front moves into each cloud, creating a highly compressed region near the plane of symmetry. Gas crosses each shock front at a speed slightly less than $V / 2$ and is adiabatically heated to a temperature such that its isothermal sound speed is roughly $w \approx 0.2 \mathrm{~V}$, i.e., to a temperature $T \approx\left(10^{6.5} \mathrm{~K}\right)$ $\left(V / 1000 \mathrm{~km} \mathrm{~s}^{-1}\right)^{2}$. As the shock moves away from it, this hot gas cools by emitting (mostly) ionizing photons, while remaining almost at constant pressure. The ionizing photons create two photoionized regions: a region in the dense cooled material near the collision plane, and a less dense region in gas which has not yet passed through the shock. Daltabuit, MacAlpine, and Cox (1978) have made specific calculations of situations like this. They enumerate five distinct zones, beginning in the undisturbed parts of a cloud and moving toward the collision plane of symmetry: A, undisturbed material which may be un-ionized; $\mathrm{B}$, the photoionized region approaching the shock; $\mathrm{C}$, the hot shocked gas; $\mathrm{D}$, the dense postshock photoionized region; and E, a very dense $\mathrm{H}^{0}$ region near the plane of symmetry. Suppose that we very crudely represent the shape of the ionizing spectrum of zone $\mathrm{C}$ as $F(\varepsilon) \sim \exp \left(-\varepsilon / k T_{C}\right)$, where $T_{C}$ is perhaps half the maximum temperature behind the shock. Then it is easy to estimate that $U_{\mathrm{H}} \sim\left(10^{8} \mathrm{~cm} \mathrm{~s}^{-1}\right)$ $\left(V / 1000 \mathrm{~km} \mathrm{~s}^{-1}\right)$ in zone $\mathrm{B}$, and $U_{\mathrm{H}} \sim\left(10^{5} \mathrm{~cm} \mathrm{~s}^{-1}\right)(\mathrm{V} /$ $\left.1000 \mathrm{~km} \mathrm{~s}^{-1}\right)^{-1}$ in zone $\mathrm{D}$, except that the last value must be increased if magnetic fields limit the compression of gas in zone D. If we want $U_{\mathrm{H}} \sim 10^{8} \mathrm{~cm} \mathrm{~s}^{-1}$ and $n_{e}$ $\sim 10^{9.5} \mathrm{~cm}^{-3}$ as in the simpler photoionized models, then we might consider either zones $B$ in collisions of dense clouds at $V \sim 1000 \mathrm{~km} \mathrm{~s}^{-1}$, or zones $\mathrm{D}$ in collisions of larger, less-dense clouds at lower speeds. The former possibility gives higher maximum temperatures, $T \sim 10^{6.5} \mathrm{~K}$. The real spectrum of a hot shocked zone $\mathrm{C}$ is not a simple continuum, but also includes ionizing emission lines and "edges"; see Cox and Daltabuit (1971), Raymond and Smith (1977), Daltabuit, Cox, and MacAlpine (1978), and Shull and McKee (1979). Note, by the way, that the energy source is largely shifted to cloud motions in this type of model, and rapid ultraviolet variations are not to be expected-because many small clouds are presumably colliding at any time, and also because of light-travel-time arguments. The idea of "cloud collisions" may be an idealized rep- resentation of a complex gas-dynamical situation, with turbulent motions and numerous shock fronts. Some of the highest-ionization emission lines, particularly [Fe $\mathrm{X}] \lambda 6374$ and [Fe XI] $\lambda 7892$ (Grandi, 1978) may arise in shock-heated gas.

Krolik, McKee, and Tarter (1978) have pointed out that radio-frequency waves can heat the gas. In photoionized regions, the most important radio absorption process is free-free (bremsstrahlung) absorption, whose relevant coefficient per unit volume is proportional to $n_{e}^{2} \lambda^{2}$. A photoionized region like those discussed in Sec. III is practically transparent at short wavelengths $\lambda \lesssim 0.01 \mathrm{~cm}$, absorbs much of the radiation at $\lambda \sim 0.1 \mathrm{~cm}$, and is quite opaque for $\lambda \gtrsim 1 \mathrm{~cm}$. This means that radiation in the wavelength range $\lambda \sim 0.1$ to $1 \mathrm{~cm}$ heats the photoionized regions. Even quasars which are "strong" radio sources have only small fractions of their luminosities in this range (e.g., see Elias et al., 1978; and Fig. 10 of Oke, Neugebauer, and Becklin, 1970), and most quasars have relatively modest radio luminosities; so this is not likely to be a very important effect. However, the radiation at longer wavelengths, $\lambda \gtrsim 1 \mathrm{~cm}$, is absorbed in such a small amount of gas that it may lead to the formation of a hot, lower-density "corona" adjoining each dense photoionized region. It is therefore conceivable that [Fe XI], [FeX], OVI, and $\mathrm{NV}$ emission may be enhanced by radio-frequency heating. If the "coronal" temperature rises too high, thermal instability occurs and the gas may become very hot indeed. Then other low-frequency processes also become important, for example, the induced Compton effect (Levich and Sunyaev, 1970). A hot, rarefied, transparent medium, roughly in pressure equilibrium with photoionized clouds, may result. Krolik, McKee, and Tarter have suggested that " $B L$ Lacertae objects" lack dense gas clouds because of very strong radio-frequency heating.

Collin-Souffrin et al. (1979) have proposed that the Fe II lines originate in dense gas which is beyond the reach of most ionizing photons, but which is heated by some unspecified process. This hypothetical gas is hot $(T \sim 20000 \mathrm{~K}$ ?) but is so dense that it is not thoroughly ionized-perhaps the atmosphere of an accretion disk is involved. The main objection to this idea, perhaps, is that it is a special explanation for just the Fe II emission and does not help to explain other spectral features.

\section{Nonthermal emission lines?}

An unusual model was proposed by Ptak and Stoner $(1973 \mathrm{a}, \mathrm{b})$, who considered streams of "suprathermal" ions (e.g., $100 \mathrm{keV}$ protons) moving through ambient gas which is mostly $\mathrm{H}^{\circ}$. Charge exchange and other interactions occur, and the radially streaming ions are said to produce broad emission lines like those of a quasar or Seyfert nucleus. Several solar masses per year must flow outward in the streams, but the ratio of streaming particles to ambient gas must be small enough to avoid ionizing and blowing away the latter. Stoner, Ptak, and Ellis (1974) calculated some asymmetric line profiles in this model, which matched certain observed profiles; other aspects or modifications were discussed by MacAlpine (1974), Ptak and Stoner 
(1975, 1977), Mergenthaler (1975), and Kimmer (1976). Katz (1975) criticized this model on several grounds: emission by the ambient gas should produce intense narrow cores in at least some of the lines, some observed spectra have asymmetric line profiles which do not match those calculated by Stoner, Ptak, and Ellis (cf. Boksenberg et al., 1975; Osterbrock and Koski, 1976), and the required geometry seems rather special and therefore implausible. Stoner and Ptak (1977) have replied that the narrow emission line "cores" may be trapped in the neutral ambient gas, and that modifications to the model can cure other difficulties. Ptak and Stoner (1976) predicted that the Fe II lines, being produced in the ambient gas, should be narrower than the hydrogen lines; but according to Phillips (1978a), this is not so. Strittmatter and Williams (1976) argued against the Ptak and Stoner models on the basis of observed $\mathrm{Mg}$ II $\lambda 2798$ widths and remarked that these models represent attempts to explain line profiles quantitatively and line intensities qualitatively, even though line intensities are better known than profiles and are perhaps less influenced by geometrical details.

The suprathermal ion streaming models are unattractive because they invoke several arbitrary, and in some respects implausible assumptions, without achieving a correspondingly detailed success in explaining observations. Ionizing photons, which we know to be present, are not really included; the streams of suprathermal ions are postulated arbitrarily; even if such streams exist, it is very surprising if they move through neutral gas in an orderly manner (presumably along magnetic field lines) without dramatically heating and ionizing the gas; some special geometrical assumptions are required in order to obtain the calculated line profiles; and the relative line intensities are not explained in detail.

\section{E. Emission-line variability}

Many quasars' ultraviolet continua are known to fluctuate markedly, over irregular time scales of several years or sometimes one year or less. We should therefore consider time-dependent effects in the line-emitting regions.

Jura (1973) proposed to explain the weakness of the He II $\lambda 4686$ lines in $3 \mathrm{C} 273$ as a time-dependent effect. Suppose that a nebula is ionized impulsively, perhaps by a sudden, temporary gust of ionizing radiation. After the ionizing process recedes, the gas begins to recombine and to cool. Because recombination is faster for doubly ionized helium than for hydrogen, after a certain time there are few $\mathrm{He}^{++}$ions left while the gas is still mostly $\mathrm{H}^{+}$and $\mathrm{He}^{+}$; then the $\mathrm{He}$ II $\lambda 4686$ recombination line becomes weak compared with $H \beta$. There are several good arguments against this scenario. First, it would almost preclude the existence of enough $\mathrm{C}^{3+}, \mathrm{N}^{4+}$, and $\mathrm{O}^{5+}$ ions to explain the observed $\mathrm{C}$ IV, $\mathrm{N} \mathrm{V}$ and $O$ VI emission, during the time when He II $\lambda 4686$ is weak. Low temperatures would also be entailed (because cooling is initially faster than hydrogen recombination), making it even more difficult to explain highexcitation lines. And the time scales do not make sense. In a gas with $n_{e} \approx 10^{9.5} \mathrm{~cm}^{-3}$, for example, the characteristic recombination time for hydrogen is only
$10^{3} \mathrm{~s}$; unless the actual densities are many orders of magnitude smaller than we have supposed, each small cloud or filament of gas can adjust its ionization structure quickly enough, compared with continuum variations, to be practically in ionization and thermal equilibrium at all times. See Bahcall, Kozlovsky, and Salpeter (1972), Shields and Oke (1975), and Boksenberg and Netzer (1977). ${ }^{11}$

If we could see an individual photoionized cloud near a quasar, its appearance at any time would depend upon the ionizing luminosity of the hypothetical central object at a somewhat earlier time; an obviously relevant parameter is the total light-travel time from the central source to the cloud and then to us. The ensemble of dense photoionized regions associated with a quasar must have an overall size scale and therefore a minimum possible time scale for variability. As suggested in preceding sections, the likely size scales, derived from preferred values of $U_{H}, n_{e}$, and ionizing luminosities, range from a light-year (for a rather modest Seyfert nucleus) to hundreds of light-years (for a very luminous quasar). These estimates are not seriously inconsistent with any reliable observations that we know of. (Cf. Bahcall et al., 1972.)

Early in the history of observations of quasars, $\mathrm{Mg}$ II emission was thought to have varied in the spectrum of one quasar (Burbidge and Burbidge, 1966; Dibai and Esipov, 1967; Wampler, 1967), but this is now thought to have been an error, perhaps related to continuum variations (cf. Pfleiderer and Grewing, 1967). Generally, when a quasar's continuum varies within a few years, its emission-line intensities remain fairly steady; this was suspected at an early date (see Sandage et al., 1966; Kinman et al., 1966; Oke, 1967) and has since been noted occasionally (e.g., see Shields, Oke, and Sargent, 1972; Osmer and Smith, 1977b) although systematic data are scarce. For the moment, at least, we are observationally justified in believing that a luminous quasar's line-emitting clouds are spread over a region which is considerably more than a light-year across.

On the other hand, emission lines have been observed to change, sometimes in time scales of much less than a year, in the spectra of a few Seyfert nuclei: see Andrillat and Souffrin (1967), Pastoriza and Gerola (1970), Andrillat (1971), Tohline and Osterbrock (1976), Boksenberg and Netzer (1977), and Phillips (1978a). The most disturbingly rapid changes-over times of the order of 10 days - have been reported by Lyutyi (1977) but have not been completely confirmed. Collin-Souffrin (1978) has asserted that these fluctuations

\footnotetext{
${ }^{11}$ The idea of nonequilibrium ionization suggests a modified type of photoionization model. Imagine a cloud being "evaporated" by ionizing photons; i.e., a steady flow of gas becomes ionized and heated as it passes through the ionization front, and thereafter expands and dissipates while being illuminated by ionizing radiation. This can in principle be a nearly steadystate situation, wherein the ionization and thermal structures are locally out of equilibrium because the gas is not stationary. In practical cases, however, the departures from local equilibrium are likely to be small, being measured by the small ratio $w / U_{\mathrm{H}}$ (where $w$ is the speed of sound).
} 
constitute evidence against photoionization models, but this is not very clear. The Seyfert nuclei in question are less luminous than quasars, so with our standard assumptions, their emission regions should have overall size scales of the order of one light-year; it would be easy to imagine asymmetric distributions of gas which would permit variations in much less than a year. Also, we have no assurance that these particular objects are exactly like quasars - for example, the relevant electron densities may be larger than those in quasars, allowing more compact arrangements with smaller light-travel times. An interesting aspect of the observations is a tendency for the $H \alpha / H \beta$ intensity ratio to become larger as the $H \beta$ intensity decreases; in other words, $H \alpha$ seems to vary less dramatically than $H \beta$. (Does this mean, according to the discussion in Sec. IV.C and especially Fig. 9, that $\tau_{H \alpha}>100$ ?)

If a quasar's photoionized line-emitting regions are dispersed in space so that there is a considerable dispersion in light-travel times, then the observed line spectrum is related to some appropriate average luminosity of the continuum source. Recalling comments made in Sec. V. G about correlations between observed luminosities and emission-line-to-continuum ratios, suppose that we were to plot these characteristics for a large ensemble of quasars, in the manner of Baldwin (1977b). There would be a statistical tendency for the instantaneously highest-luminosity objects to be above their average luminosities, and this would help to explain the observed effect. It is also possible to invent other ways in which continuum fluctuations, over time scales of many years, would induce this type of correlation, especially if some of the line-emitting gas is optically thick and some is optically thin to ionizing photons.

\section{GAS MOTIONS AND DYNAMICS; LINE PROFILES}

\section{A. Generalities}

A truly "self-consistent" photoionization model would include some sort of dynamical equilibrium within each ionized zone, as well as thermal and ionization equilibria. There should be an explanation for the character istic gas pressures and values of $U_{\mathrm{H}}$ which have been mentioned in Secs. III, IV, and V. And there should also be explanations of the characteristic emission linewidths and profiles which are observed. Unfortunately, relations between these matters and available observational data are so indirect that unique conclusions cannot be drawn from the data-this situation is even murkier than the ionization problems discussed earlier. Papers on quasar-related dynamical problems tend to be quite detailed, specific-and idealized!

At a very simple, order-of-magnitude level, we can guess several interesting parameters for a quasar's broad-line emission regions: the photoionized gas pressure $\left(P_{\text {gas }} \sim 10^{-2}\right.$ dyn $\left.\mathrm{cm}^{-2}\right)$; either the total radiation pressure or the ionizing radiation pressure $\left(P_{\text {rad }} \sim 10^{-2}\right.$ or $10^{-3}$ dyn $\left.\mathrm{cm}^{-2}\right)$; the local velocity dispersion within each photoionized region, including thermal motions and subsonic turbulence $\left(w \sim 15\right.$ or $\left.20 \mathrm{~km} \mathrm{~s}^{-1}\right)$; and the overall velocity dispersion $\left(v \sim 10^{3}\right.$ to $\left.10^{4} \mathrm{~km} \mathrm{~s}^{-1}\right)$. This last parameter is indicated by the observed linewidths mentioned in Sec. I, $\Delta \lambda / \lambda \sim 2 v / c{ }^{12}$ A few relevant comments, about the gas pressures, a likely confining "intercloud medium," and the total implied energies, have already been made at the end of Sec. III. B. These comments need only slight revision if dust is important as discussed in Sec. V. Now some remarks about the velocity dispersion $v$ are in order. It seems likelythough not yet provable-that $v$ is related to gravitational (orbital or escape) speeds in the emission regions. This is obvious if the emission regions are orbiting or falling into a central mass; it is also worth considering if the line-emitting gas has been ejected from a central locality. If $M$ is the mass within radius $r$, and $M_{s}$ denotes one solar mass, the escape speed at radius $r$ is

$$
v_{\text {esc }} \approx\left(1700 \mathrm{~km} \mathrm{~s}^{-1}\right)\left(\frac{M / 10^{8} M_{S}}{r / 1 \text { light-year }}\right)^{1 / 2} .
$$

If we adopt characteristic values $U_{\mathrm{H}} \sim 10^{8.2} \mathrm{~cm} \mathrm{~s}^{-1}$ and $n_{e} \sim 10^{9.5} \mathrm{~cm}^{-3}$, as discussed previously, and if $L \mathrm{de}-$ notes the total luminosity of a quasar, then (very crudely) $r \sim(3$ light-years $)\left(L / 10^{46} \mathrm{erg} \mathrm{s}^{-1}\right)^{1 / 2}$ for the broad-line-emitting regions; so

$$
v_{\text {esc }} \sim\left(10^{3} \mathrm{~km} \mathrm{~s}^{-1}\right)\left(\frac{M}{10^{8} M_{S}}\right)^{1 / 2}\left(\frac{L}{10^{46} \mathrm{erg} \mathrm{s}^{-1}}\right)^{-1 / 4} .
$$

Masses of $10^{8}$ to $10^{11} M_{S}$ seem consistent with the observed linewidths.

There is another relation involving $M$ and $L$, known as the "Eddington limit." Make the fashionable supposition that much of the luminosity of a quasar derives from gravitational accretion onto one or more compact objects (most likely a central massive black hole). Some outward momentum is transferred from escaping radiation to the infalling material. If the luminosity is sufficiently large relative to the central mass, this radiation pressure strongly impedes the accretion. But

\footnotetext{
${ }^{12}$ Shklovsky (1965) proposed that quasars' linewidths are actually due to scattering by free electrons, so that $v$ is related to electron velocities in a hot, quiescent medium; Burbidge et al. (1966) even suggested that this would explain observed differences between permitted and forbidden linewidths. Weymann (1970), Mathis (1970), and Auer and Van Blerkom (1972) calculated some related line profiles. Today, we do not believe that scattering by free electrons is generally important; this is mainly because photoionization analyses lead to models which are optically thin to such scattering $\left[U_{\mathrm{H}}\right.$ $\ll 10^{11} \mathrm{~cm} \mathrm{~s}^{-1}$; see comment following Eq. (2.5)], and partly because the idea of electron scattering provides no advantage in explaining the observed line profiles as we know them today. Moreover, one can show that a simple model, with a single central source surrounded by a hot gas, cannot meet all of the following conditions which must obtain before electron scattering can become important: the gas must be optically thick to scattering, but it cannot absorb radio waves too strongly, and the overall dimension must be small enough to allow scattered continuuum radiation to vary within a year or so. Note, also, that one cannot use resonance scattering to weaken the first condition, because the observed semiforbidden lines are as broad as the resonance lines. With the possible exceptions of peculiar high-excitation lines from nonphotoionized gas in a few special objects (C IV in NGC 4151?), electron scattering is unlikely to be important regarding the prominent linewidths.
} 
the luminosity depends upon the accretion rate; so there is a limit to the luminosity-to-mass ratio $L / M$. This limit depends upon the opacity of the infalling gas, and for ionized gas is highest if the gas is hot and rarefied so that scattering by free electrons is the dominant process. (Obviously, the dense line-emitting regions do not contain such gas.) Then the "Eddington limit" is

$$
L \lesssim\left(10^{46_{\circ} 2} \operatorname{erg~s}^{-1}\right)\left(M / 10^{8} M_{S}\right) .
$$

(Many subtleties can occur here, especially if there are optically thick blobs or special geometrical arrangements. We feel obliged to mention the Eddington limit because it is so often invoked, but the interested reader may invent ways of evading it.) If (8.3) is valid, then comparison with (8.2) gives

$$
v_{\mathrm{esc}} \gtrsim\left(800 \mathrm{~km} \mathrm{~s}^{-1}\right)\left(\frac{L}{10^{46} \operatorname{erg~s}^{-1}}\right)^{1 / 4} \text {. }
$$

It is both easy and consistent to imagine that a quasar and its line-emitting regions have these characteristics: (i) The luminosity is not very far below the Eddington limit. (ii) The central mass is $10^{8}-10^{12} M_{S}$, roughly proportional to the luminosity. (iii) The observed velocity dispersion $v$ is comparable to $v_{\text {esc }}$ for the line-emitting regions.

Most imagined pictures of quasars include several components: A massive central object, a dense gaseous disk, a dense cluster of stars and related ojects, a rarefied medium (either hot thermal gas or nonthermal particles and fields), and dense clouds of lineemitting gas. Some of these components may not be easily distinguishable, and some may be unnecessary. Gas may be liberated when stars are disrupted, either tidally by the black hole, or in stellar collisions, or in supernova explosions; or gas may be falling in from a surrounding galaxy. Some gas may fall into the disk and spiral into the black hole; some may be confined in clouds at the emission-line-region densities, $n_{e} \sim 10^{9.5}$ $\mathrm{cm}^{-3}$; some may become part of a low-density hot medium. The low-density gas may be blown outward in a "wind," by radiation pressure or by relativistic particles and fields, or by its own pressure; this wind may carry line-emitting clouds along with it, or may condense into clouds or sheets or filaments because of instabilities. The hypothetical disk may contain the emission-line gas or may be its source. Shock waves, and sources of energetic particles and electromagnetic fields, can occur in many places among the various components. Many authors have rung the changes in this bewildering belfry since the mid-1960s.

Because the observational data are obviously inadequate to give us much more than we have already discussed in Secs. III-V, one is tempted to try to choose among the various schemes by looking for theoretical inconsistencies or instabilities. Occasionally an author claims that some intricate scenario is inevitable, but assertions of this type have often turned out to be wrong. We shall restrict our attention to a few aspects of the problem.

\section{B. Acceleration of gas-mainly by radiation pressure}

Some hot stars are known to eject material in continuous "stellar winds" which are driven largely by ra- diation pressure. This fact inspired Mushotzky, Solomon, and Strittmatter (1972) to suggest that radiatively driven winds also occur around quasars; Tarter and McKee (1973) then derived better estimates of the rates of momentum transfer. Many papers on this subject have appeared subsequently. Mathews (1974) and Kippenhahn, Perry, and Röser (1974) calculated the accelerations of optically thin, photoionized clouds (see also Opher, 1974a). Blumenthal and Mathews (1975) employed a few simple assumptions to show that an ensemble of such clouds, constantly replenished near the center of activity, might produce emission-line profiles which resemble those of quasars.

However, Williams (1972) pointed out that isolated, photoionized clouds must be disrupted by internal pressures (including trapped resonance photons) before large velocities can be attained; see also Weymann (1973), Noerdlinger and Rybicki (1974), and McKee and Tarter (1975). Because of the nature of the ionization equilibrium, the radiative momentum absorbed per unit volume is proportional to $n_{e}^{2}$, so the acceleration is proportional to $n_{e}$. High densities are required for reasonable accelerations, but isolated, compact, high-density clouds, expanding at roughly their internal sound speeds, dissipate very quickly. A conceivable escape from this difficulty - if instabilities are not too bad-is to suppose that each gas cloud is confined by a surrounding medium; Blumenthal and Mathews (1975) invoked such a medium in their line-profile calculations. Mathews (1976), Mathews and Blumenthal (1977), and Krolik (1979) have discussed some relevant gas-dynamical instabilities. Suppose that a photoionized cloud is radiatively accelerated through a low-density medium whose pressure is sufficient to confine the cloud (except for instabilities). If the cloud is optically thin, a Rayleigh-Taylor-like instability develops at the leading edge. However, according to Mathews and Blumenthal, there are mitigating effects, so that as the instability grows to a nonlinear stage, its growth rate may slow down; the practical result is left in doubt. A cloud with an appreciable but moderate optical depth to ionizing photons $\left(\tau_{\mathrm{H}} \sim 20\right.$ ?) can be stable. A thick cloud, which contains an $\mathrm{H}^{+} / \mathrm{H}^{0}$ ionization front $\left(\tau_{\mathrm{H}} \geq 50\right)$, is quite unstable at the ionization front. A cloud which is accelerated by surface forces (i.e., by the confining medium) is also very unstable. Mathews and Blumenthal suggest that flat ("pancake-shaped") photoionized clouds with moderate optical depths may produce most of the important emission lines. This seems inconsistent with some of our comments in Secs. III-V, where we suggested that large optical depths, $\tau_{\mathrm{H}}>50$, are necessary for several reasons. However, the dynamically critical thickness depends upon a radiation pressure gradient, and if $x$-rays are more important than Mathews and Blumenthal supposed, the discrepancy may be reduced.

Kippenhahn, Mestel, and Perry (1975) developed solutions for a continuous "quasar wind," driven by radiation pressure but influenced by internal gas pressure. The hypothetical wind is mostly rarefied, optically thin, and practically invisible, but according to Mestel, Moore, and Perry (1976) and Kippenhahn (1977), an ionization instability may cause density 
waves to form, leading to shock fronts and perhaps condensations. These authors intended to explain absorption lines in quasars' spectra, and it is not clear how their results may be related to the emission-line regions or to the results of Mathews and Blumenthal. See also Kippenhahn (1978). Burbidge and Perry (1976) attempted to derive quasars' masses from ideas about radiation-driven winds; considering that some of their assumptions and parameters can easily be modified, their results are consistent with conventionally suspected values, $10^{8}$ to $10^{11}$ solar masses. This does not prove that the winds exist, of course.

Radiation-driven clouds and winds have been popular largely because they are susceptible to idealized, mathematically well-defined treatment. We suspect, by analogy with supernova remnants and diffuse nebulae in our Galaxy, that nonradiative pressures - the "confining medium," in fact-may dominate the dynamics of quasars' emission regions. Eilik and Caroff (1976) and Rowe, Stoner, and Ptak (1978), have considered the possible "volume forces" (rather than surface pressure) that would result from nonthermal particles streaming through the gas, but little attention has been given to the possible role of magnetic or electromagnetic fields (see Arons, Kulsrud, and Ostriker, 1975, for possible inspiration or discouragement). Marscher (1978) has discussed acceleration of clouds by repeated explosion waves.

\section{Emission-line profiles}

A few years ago, it was hoped that quasars' line profiles would provide decisive information about the emis sion regions. Unfortunately, various contradictory explanations of the observed profiles have all been partly successful. There are four basic facts to be explained:

(i) In a typical quasar's spectrum, the broad lines all have practically the same shape and relative width. For example, the profiles of C IV $\lambda 1549$ and C III ] $\lambda 1909$ can usually be superimposed (see Fig. 13, taken from Baldwin and Netzer, 1978). The $L \alpha$ profile is trickier because it usually has absorption lines on its shortwavelength side and $\mathrm{N} \mathrm{V} \lambda 1240$ emission on its longwavelength side; but the data are usually consistent with the idea that intrinsic $L \alpha$ and $N \mathrm{~V} \lambda 1240$ profiles are like that of C IV $\lambda 1549$. The broad Balmer and helium lines are probably similar in most cases. [See Baldw in $(1975,1978)$, Osterbrock (1977), and Osmer and Smith $(1976,1977 a)$.] Most quasars' broad linewidths $c \Delta \lambda / \lambda$ (where $\Delta \lambda=$ "FWHM," "full width at halfmaximum") are between $10^{3}$ and $10^{4} \mathrm{~km} \mathrm{~s}^{-1} ; 5000 \mathrm{~km} \mathrm{~s}^{-1}$ is a good typical value.

(ii) However, some objects produce broad lines which are not all alike. In two very luminous quasars, PHL 957 and $B 1225+31.7$, the $C$ IV line is narrower than the C III ] and $L \alpha$ lines (Coleman et al., 1976; Baldwin and Netzer, 1978). In several objects, including the peculiar Seyfert galaxy NGC 4151 , the He I $\lambda 5876$ line is known to be wider than $H \beta$ (Boksenberg et al., 1975; Osterbrock and Koski, 1976). Mg II $\lambda 2798$ may occasionally be wider than $H \beta$ (Grandi and Phillips, 1978). $H \alpha$ and $H \beta$ have different widths in some cases (Osterbrock, 1977).

(iii) In most quasars' spectra, each broad-line pro-

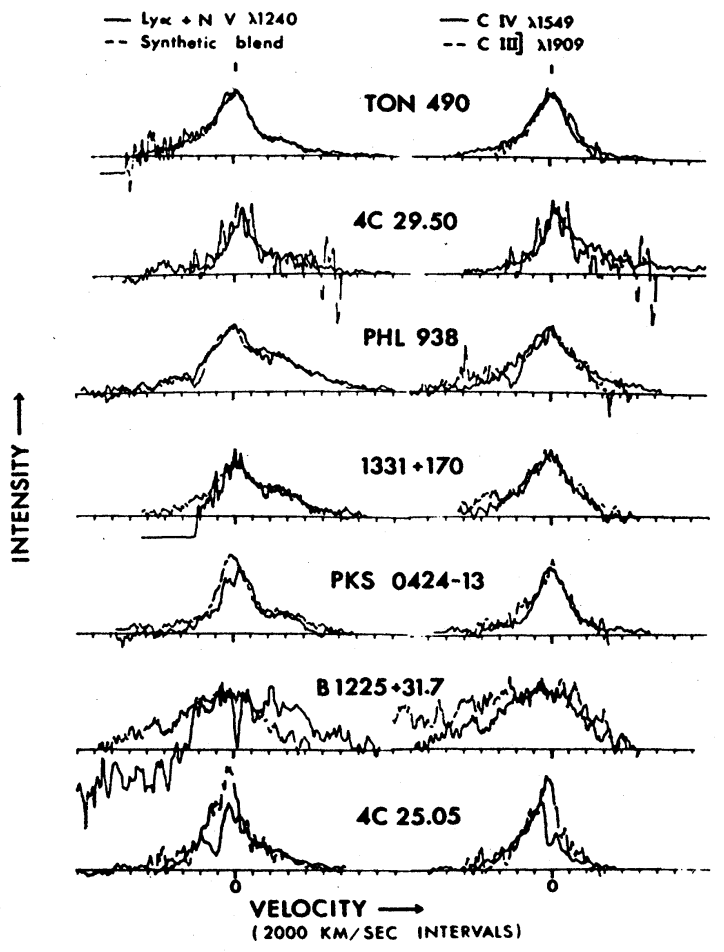

FIG. 13. The profiles of several lines in high-red-shift quasars' spectra. See Baldwin and Netzer (1978) for details; the point is that the $L \alpha, \mathrm{C} \mathrm{IV}$ and $\mathrm{C}$ III lines all seem to have the same intrinsic profile, if one allows for absorption on the short-wavelength side of $L \alpha$. Note, however, that the particular luminous quasar B $1225+31.7$ is an exception.

file is symmetric and rather triangular, with slightly concave sides ("wings"). A good example, despite many superimposed absorption lines, is shown in Fig. 14, taken from data obtained by Boksenberg et al. See also Osmer and Smith (1977a), Baldwin and Netzer (1978), Osterbrock (1977), and Fig. 13. The peak of each line probably includes emission from the narrow-line regions.

(iv) But in some objects' spectra, the profiles are asymmetric. The quasar PHL 1093 is one good example (Baldwin, 1975); see also Ulrich (1972b), Disney (1973), Osmer, Smith, and Weedman (1974), and Miley and Miller (1979). Many Seyferts also have asymmetries (Osterbrock, 1977). In most such cases, the line profiles resemble triangles with unequal sides, in such a way that the lines" "centers of gravity" are at longer wavelengths than their peaks. Where perceptible narrow lines are superimposed, they usually appear near the peaks of the asymmetric broad lines.

Shields (1978) has discussed some of the questions raised by these facts. If the emitting regions are simply rotating in a disk or falling inward, then their characteristic velocities should depend upon radial position, $v \sim r^{-1 / 2}$; the broad wings of each line should be formed in the innermost parts of the configuration. But the line ratios produced at any radius depend upon $U_{\mathrm{H}}$; therefore, in order for $L \alpha, \mathrm{C} \mathrm{IV} \lambda 1549$, and C III ] $\lambda 1909$ to have similar profiles, $U_{\mathrm{H}}$ must be nearly independent of radius. Shields suggests that this is additional evi- 


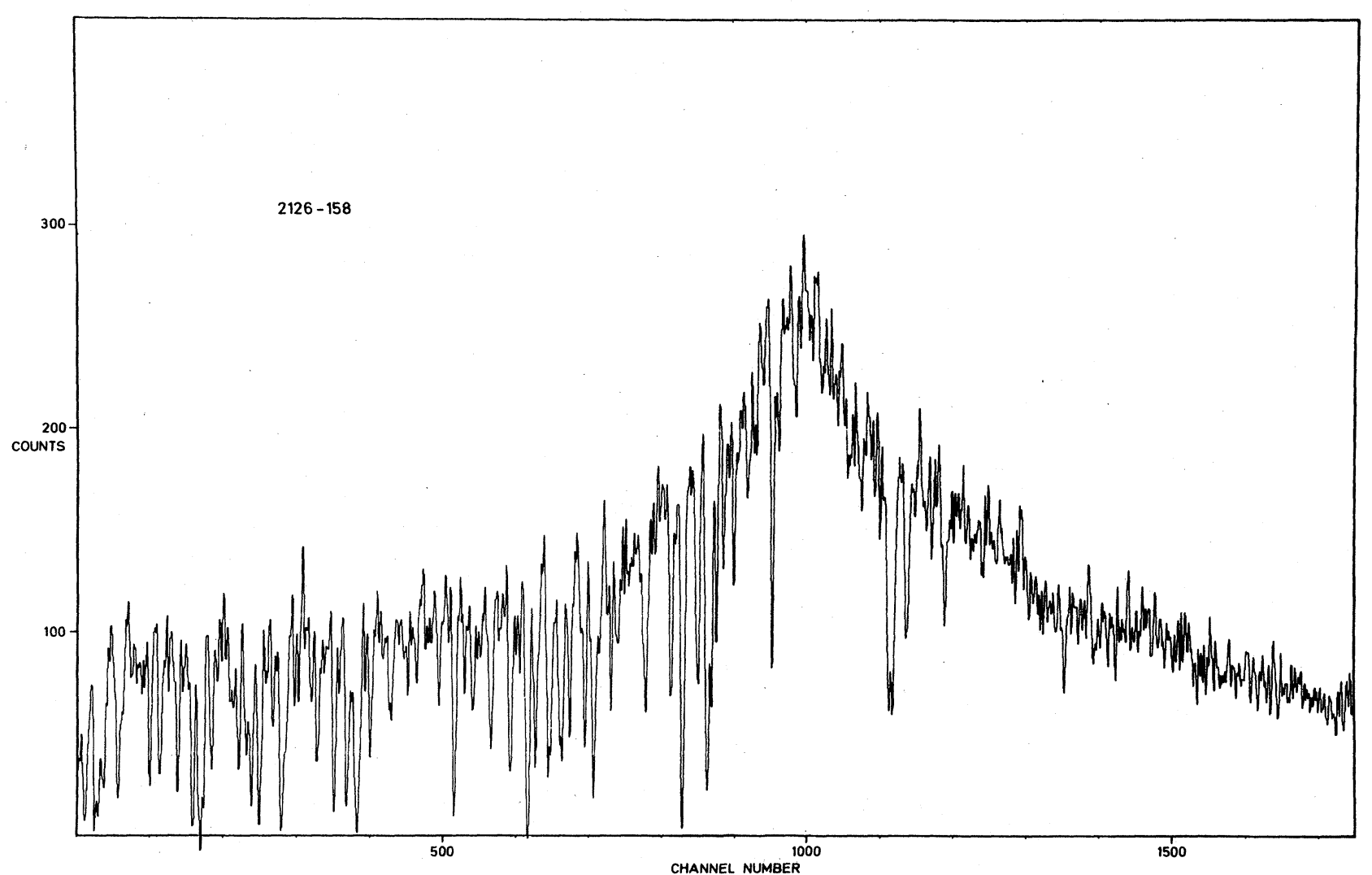

FIG. 14. The profile of a particular quasar's $L \alpha$ line, obtained with high spectral resolution by Boksenberg's group. The horizontal width of the figure corresponds to a rest-wavelength interval of about $150 \AA$. This spectrum has many narrow $L \alpha$ absorption lines, probably not directly related to the broad emission line; note that they tend to fall along the short-wavelength side of the emission line.

dence for something that we must already suspect: there is a physical connection between the gas and radiation densities which causes them to have a particular ratio. However, as Shields also notes, in this picture the gas densities that produce the line wings should be very much greater than those which give the centers of the lines; this makes it difficult to explain how $\left.\mathrm{C}_{\mathrm{III}}\right]$ $\lambda 1909$ can have broad wings while narrower $\left[\mathrm{O}_{\mathrm{III}}\right] \lambda 5007$ emission is weak. The relation $v \sim r^{-1 / 2}$ is probably oversimplified; but the idea that velocity dispersion is correlated with radial position may be useful in partially explaining fact (ii) above. As we mentioned in Sec. IV. D, HeI $\lambda 5876$ is enhanced more strongly if $F_{\mathrm{H}}$ is larger, presumably at smaller values of $r$; so it is qualitatively reasonable for He I $\lambda 5876$ to be wider than $H \beta$ in some gravitationally bound cases (Netzer, 1978).

Most writers on the subject have agreed with Shields (1978) in preferring models wherein the gas flows outward. The role of gravity in such a model is not completely obvious. Since it is difficult to believe that the central mass of a quasar can be very much less than the values mentioned earlier, it appears that the hypothetical outflow velocities are not very much greater than $v_{\text {esc }}$ at the source of the outflow. (This is reminiscent of stellar winds.) Blumenthal and Mathews (1975) showed that a particular model with radiatively accelerated clouds would produce symmetric line profiles much like those which are observed in most cases.
However, this model is unappealing because each of its clouds is optically thin. The information conveyed by a standard symmetric line profile is like that given by the standard set of line intensities: some finite region in "parameter space" is allowable. For the line profiles, if we assume spherical or axial symmetry, the important parameters are the distributions of $n_{e}$, $U_{\mathrm{H}}, \tau_{\mathrm{H}}$, and covering factors, as functions of velocity dispersion $v$. Radial position $r$, although important, affects observable quantities only indirectly.

Asymmetric profiles have been interpreted by Capriotti, Foltz, and Byard (1979) and Ferland, Netzer, and Shields (1979) as evidence that the line-emitting gas is moving outward. These authors discuss ensembles of thick clouds, moving radially outward. Because of resonance and Balmer-line scattering and/or internal dust, emission from the photoionized part of each cloud tends to escape through the illuminated inner edge of the cloud. Looking at an expanding array of clouds from a distance, we therefore see the clouds on the far side best; since those clouds are moving away from us, the observed asymmetric line profiles can be explained-qualitatively, at least, and perhaps also quantitatively. But if this is true, why do most quasars have symmetric emission lines? Perhaps most quasars' emission regions are not very thick (Mathews and Blumenthal might prefer this view); or perhaps the velocity dispersions in most quasars have substantial 
nonradial components. Note, also, that much depends upon the viewing angle if the configuration is not spherically symmetric. For example, gravitational and transverse Doppler red shifts can modify the line profiles for a disklike configuration seen "face-on" (Netzer, 1977b).

As mentioned in Sec. VII. D, Stoner, Ptak, and Ellis (1974) proposed a quite different explanation for some asymmetric line profiles.

\section{More remarks about certain parameters}

Unless we have been misled in Secs. III, IV, and V, many quasars, spanning a wide range in luminosity, all seem to have line-emitting regions with similar values of $U_{\mathrm{H}}$ and $n_{e} \cdot U_{\mathrm{H}}$ is probably just above $10^{8} \mathrm{~cm} \mathrm{~s}^{-1}$, while $n_{e}$ is probably just below $10^{10} \mathrm{~cm}^{-3}$. The corresponding thermal energy density (or pressure) in the photoionized gas is just above $0.01 \mathrm{erg} \mathrm{cm}^{-3}$, and the total radiation energy density is not much less. Comparable energy densities have sometimes been mentioned for relativistic particles and electromagnetic fields which produce nonthermal emission in some quasars [e.g., see Jones et al. (1974) and Burbidge et al. (1974), as well as references cited by Burbidge and Burbidge (1967)]. Furthermore, one can imagine a thermal "intercloud medium" with the same energy density (pressure) and whose speed of sound is comparable to the observed velocity dispersions. ${ }^{13}$ These order-of-magnitude numbers are vaguely satisfying, in a speculative way, but we should try to explain them. In particular: why are the gas and radiation pressures comparable in the line-emitting regions, and what is special about the value $0.01 \mathrm{erg} \mathrm{cm}^{-3}$ ? The idea of radiation pressure balancing gas pressure, while obvious, is made difficult by instabilities - see the references cited in Sec. VIII. B.

Shields (1978) has mentioned some of the many possible starting points for answers to these questions. For example, suppose that ionizing radiation illuminates a gravitationally bound atmosphere from above. If the radiation pressure forces exceed gravity forces in the photoionized layers, then these layers are compressed by radiation so that radiation and gas pressures are comparable there, while gravity serves to stabilize the situation. If the ionizing continuum is a power law like $F(\varepsilon) \sim \varepsilon^{-1}$, then the expected value of $U_{H}$ is of the order of $10^{9}$ rather than $10^{8} \mathrm{~cm} \mathrm{~s}^{-1}$; but if the ionizing spectrum is "harder" or "flatter" as discussed in.Sec. V. F, this discrepancy is less serious. Shields speculates that the hypothetical atmosphere is on the surface of an accretion disk, but one might also consider a model in-

\footnotetext{
${ }^{13}$ The required temperature would be almost $10^{9} \mathrm{~K}$, so that $k T$ is almost $100 \mathrm{keV}$, and the density $n_{e}$ would be almost $10^{5}$ $\mathrm{cm}^{-3}$. In the usual case, such a thermal medium cannot fill the entire region of interest, because it would be optically thick to scattering by free electrons. As noted by Davidson (1972), a variation of this idea is to suppose that the re are large, not-so-hot clouds, with $n_{e} \sim 10^{5} \mathrm{~cm}^{-3}$, whose random velocities provide the overall energy density or pressurei.e., when such clouds collide they form the dense regions whose emission lines we observe.
}

volving envelopes around many stars, especially red giant stars. The situation need not be hydrostatic; there may be steady flows, or locally unsteady flows whose average properties are represented by the whole configuration.

Another interesting speculation concerns radiation densities and dust grains. As discussed in Sec. IV, radiation densities seem to be roughly the same in most quasars' broad-line-emitting regions; and as discussed in Sec. $\mathrm{V}$, these radiation densities are just adequate to heat solid grains almost to their evaporation temperatures. According to Shields (1978), perhaps this is more than a coincidence. Perhaps there is some instability that causes dense line-emitting clouds to form as soon as dust grains have condensed in an outflowing "wind," or where grains are destroyed in infalling gas. One could extend this speculation by wondering whether each photoionized region has a moderate but not excessive optical thickness for ultraviolet absorption by dust; this might set $U_{\mathrm{H}}$. We should also remember that most published radiative-acceleration models have not included dust.

Maybe there are some clues, which we do not quite understand, in the surprising geometrical parameters. The thickness $x_{\mathrm{H}}$ of each dense photoionized region is probably only $10^{11}$ or $10^{12} \mathrm{~cm}$, so that the associated time scales $x_{\mathrm{H}} / U_{\mathrm{H}}$ and $x_{\mathrm{H}} / w$ are only about $10^{3}$ and $10^{5}$ s. The total cross-section area of all of the photoionized regions, required to give the necessary "covering factor," must be $10^{36}$ to $10^{39} \mathrm{~cm}^{2}$ for a respectable quasar. If each ionized region resembled a spherical cloud, more than $10^{14}$ such clouds would be required! This is why sheetlike photoionized regions are appealing. Three obvious types of sheets may occur (i) near shock fronts, (ii) on the surfaces of large, very dense $\mathrm{H}^{0}$ clouds, or (iii) on the surface of a hypothetical gaseous disk. The latter two possibilities are not entirely distinct, because a disk is likely to be inhomogeneous. The idea of an assembly of clouds may be topologically wrong; shock fronts and very hot or nonthermal gas may from "bubbles" or "tunnels" in a cooler gaseous medium, and the photoionized gas may form the surface of these bubbles or tunnels.

Throughout this whole problem runs the difficulty that we do not really understand the dynamical behavior of interstellar clouds, nebulae, and supernova remnants in our own Galaxy. Many "theoretical" papers about quasars have been based on elaborate scenarios. Geometrically detailed models, invented in the same way but applied to resolvable objects in our Galaxy, have often (usually?) been overthrown as soon as adequate observations have become possible. There is no reason to think that similarly detailed models of quasars would fare any better if observers could resolve quasars' structures.

We believe that attention should be concentrated on parameters whose values (or orders of magnitude, at least) are known without much recourse to geometrical suppositions. These parameters are $F_{\mathrm{H}}$ and $n_{e}$, their ratio $U_{H}$, their associated energy density or pressure, and their implied size scales; the characteristic velocity dispersion; perhaps the "covering factor"; the total luminosity; the relative abundances of hydrogen, 
helium, and heavier elements, and the existence or nonexistence of associated dust; and the continuum spectrum shape, and its fluctuations in time. Except for the very last item, these parameters cannot be derived without knowing about the emission lines; even the shape of the continuum spectrum may be modified by intervening dust, whose presence is detectable through its effect on emission lines. One might object that most of the parameters that we have mentioned are not directly related to the hypothetical energy sourcewhich may or may not be a massive black hole-but we reply that (i) if a "central object" does not provide us with useful, specific observable quantities, then speculations about its related small-scale details are unsatisfactory because they cannot be verified or overthrown by observations; and (ii) perhaps the most important questions about a quasar involve its formation and its persistent supply of "fuel"-both of which involve diffuse matter that is probably related to the emission regions.

\section{ACKNOWLEDGMENTS}

We began the preparation of this review at the 1978 Santa Cruz "Summer Workshop" on Quasars and Active Galactic Nuclei, and wish to thank the organizers and participants of that meeting for much inspiration, provocation, and advice. We are grateful to J. A. Baldwin, J. B. Oke, D. E. Osterbrock, and D. and B. Wills for providing some of the figures, and to many other people, including J. N. Bahcall, J. Krolik, G. M. MacAlpine, R. McCray, E. E. Salpeter, and G. A. Shields, for helpful comments. K. D. gratefully acknowledges support by the Alfred P. Sloan Foundation, and also the hospitality of the Joint Institute for Laboratory Astrophysics (Boulder), where this article was revised.

\section{REFERENCES}

Aannestad, P. A., and E. M. Purcell, 1973, Annu. Rev. Astron. Astrophys. 11, 309.

Adams, T. F., 1971, Astron. Astrophys. 12, 280.

Adams, T. F., 1972, Astrophys. J. 174, 439.

Adams, T. F., 1975a, Astrophys. J. 196, 675.

Adams, T. F., 1975b, Astrophys. J. 201, 350.

Adams, T. F., and D. W. Weedman, 1975, Astrophys. J. 199, 19.

Adams, W. M., and V. Petrosian, 1974, Astrophys. J. 192, 199.

Aldrovandi, S. M. V., and D. Pequignot, 1973, Astron. Astrophys. 25, 137; 47, 321(E) (1976).

Allen, D. A., 1976, Astrophys. J. 207, 367.

Aller, L. H., 1956, Gaseous Nebulae (Wiley, New York).

Anderson, K. S., 1970, Astrophys. J. 162, 743.

Andrillat, Y., 1971, Astrophys. Lett. 8, 161.

Andrillat, Y., and S. Souffrin, 1967, Astrophys. Lett. 1, 113.

Angel, J. R. P., H. S. Stockman, N. J. Wolf, E. A. Beaver, and P. G. Martin, 1976, Astrophys. J. Lett. 206, L5.

Angione, R. J., 1976, Astron. J. 76, 25.

Appenzeller, I., 1975, Astron. Astrophys. 38, 313.

Arons, J., R. M. Kulsrud, and J. P. Ostriker, 1975, Astrophys. J. 198, 687.

Arons, J., and D. W. Wingert, 1972, Astrophys. J. 177, 1. Auer, L. H., 1968, Astrophys. J. 153, 783.

Auer, L. H., and D. Van Blerkom, 1972, Astrophys. J. 178, 175.

Avery, L. W., and L. L. House, 1968, Astrophys. J. 152, 493.
Bahcall, J. N., 1966, Astrophys. J. 145, 684.

Bahcall, J. N., 1971, Astron. J. 76, 283.

Bahcall, J. N., 1978, Phys. Scr. 17, 229.

Bahcall, J. N., and B.-Z. Kozlovsky, 1969a, Astrophys. J, 155, 1077.

Bahcall, J. N., and B.-Z. Kozlovsky, 1969b, Astrophys. J. 158, 529.

Bahcall, J. N., B.-Z. Kozlovsky, and E. E. Salpeter, 1972, Astrophys. J. 171, 467.

Bahcall, J. N., and J. B. Oke, 1971, Astrophys. J. 163, 235.

Bahcall, J. N., and W. L. W. Sargent, 1967, Astrophys. J. Lett. 148, L65.

Bailey, M. E., and S. V. M. Clube, 1978, Nature (Lond.) 275, 278.

Baldwin, J. A., 1975, Astrophys. J. 201, 26.

Baldwin, J. A., 1977a, Mon. Not. R. Astron. Soc. 178, 67P. Baldwin, J. A., 1977b, Astrophys. J. 214, 679.

Baldwin, J. A., 1978, in Proceedings of the 1977 NATO Cambridge Summer Conference on Quasars (in press).

Baldwin, J. A., W. L. Burke, C. M. Gaskell, and E. J. Wampler, 1978, Nature (Lond.) 273, 431.

Baldwin, J. A., and H. Netzer, 1978, Astrophys. J. 226, 1.

Baldwin, J. A., H. E. Smith, E. M. Burbidge, C. Hazard, H. S. Murdoch, and D. L. Jauncey, 1976, Astrophys. J. Lett. 206, L83.

Barr, P., N. E. White, P. W. Sanford, and J. C. Ives, 1977, Mon. Not. R. Astron. Soc. 181, 43P.

Bates, D. R., and A. Dalgarno, 1962, in Atomic and Molecular Processes, edited by D. R. Bates (Academic, New York), p. 249.

Bates, D. R., A. E. Kingston, and R. W. P. McWhirter, 1962a, Proc. R. Soc. A 267, 297.

Bates, D. R., A. E. Kingston, and R. W. P. McWhirter, 1962b, Proc. R. Soc. A 270, 155.

Bearden, A. J., 1966, J. Appl. Phys. 37, 1681.

Beaver, E. A., R. Harms, C. Hazard, H. S. Murdoch, R. F. Carswell, and P. A. Strittmatter, 1976, Astrophys. J. Lett. 203, L5.

Begelman, M. C., and M. J. Rees, 1978, Mon. Not. R. Astron. Soc. 185, 847 .

Bell, K. L., and A. E. Kingston, 1967, Proc. Phys. Soc. (Lond.) 90, 31.

Bely, O., 1966, Proc. Phys. Soc. (Lond.) 88, 587.

Bely, O., J. Tully, and H. Van Regemorter, 1963, Ann. Phys. (Paris) 8, 303.

Bely, O., and H. Van Regemorter, 1970, Annu. Rev. Astron. Astrophys. 8, 329.

Bergeron, J., 1976, Astrophys. J. 210, 287.

Bergeron, J., and S. Collin-Souffrin, 1974, Astron. Astrophys. $36,27$.

Bergeron, J., and E. E. Salpeter, 1973, Astron. Astrophys. $22,385$.

Bergeron, J., and S. Souffrin, 1971 Astron. Astrophys. 14, 167.

Berrington, K. A., P. G. Burke, P. L. Dufton, and A. E. Kingston, 1977, J. Phys. B 10, 1465.

Bethe, H. A., and E. E. Salpeter, 1957, Quantum Mechanics of One-and Two-Electron Atoms (Springer, Berlin).

Black, J. H., J. C. Weisheit, and E. Laviana, 1972, Astrophys. J. 177, 567.

Blaha, M., 1969, Astrophys. J. 157, 473.

Blaha, M., 1972, Astron. Astrophys. 16, 437.

Blandford, R. D., and C. F. McKee, 1977, Mon. Not. R. Astron. Soc. 180, 343 .

Blint, R. J., W. D. Watson, and R. B. Christiansen, 1976, Astrophys. J. 205, 634 .

Blumenthal, G. R., and W. G. Mathews, 1975, Astrophys. J. 198, 517.

Boardman, W. J., 1964, Astrophys. J. Suppl. Ser. 9, 185.

Boksenberg, A., et al., 1978a, Nature (Lond.) 275, 404.

Boksenberg, A., R. F. Carswell, M. G. Smith, and J. A. J. Whelan, 1978b, Mon. Not. R. Astron. Soc. 184, 773. 
Boksenberg, A., and H. Netzer, 1977, Astrophys. J. 212, 37. Boksenberg, A., K. Shortridge, D. A. Allen, R. A. E. Fosbury, M. V. Penston, and A. Savage, 1975, Mon. Not. R. Astron. Soc. 173, 381 .

Bonilha, J. R. M., R. Ferch, P. Noerdlinger, E. E. Salpeter, and G. Slater, 1979, Astrophys. J., in press.

Bowen, I. S., 1934, Publ. Astron. Soc. Pac. 46, 146.

Bowen, I. S., 1935, Publ. Astron. Soc. Pac. 81, 1.

Bowen, I. S., 1947, Publ. Astron. Soc. Pac. 59, 196.

Breit, G., and E. Teller, 1940, Astrophys. J. 91, 215.

Brocklehurst, M., 1970, Mon. Not. R. Astron. Soc. 148, 417.

Brocklehurst, M., 1971, Mon. Not. R. Astron. Soc. 153, 471.

Brocklehurst, M., 1972, Mon. Not. R. Astron. Soc. 157, 211.

Brown, R. L., 1971, Astrophys. J. 164, 387.

Brown, R. L., 1972, Astrophys. Space Sci. 16, 274.

Burbidge, E. M., 1970, Astrophys. J. Lett. 160, L33.

Burbidge, E. M., and G. R. Burbidge, 1966, Astrophys. J. 143, 271.

Burbidge, G. R., and E. M. Burbidge, 1967, Quasi-Stellar Objects (Freeman, San Francisco).

Burbidge, G. R., E. M. Burbidge, F. Hoyle, and C. R. Lynds, 1966, Nature (Lond.) 210, 774.

Burbidge, G. R., A. H. Crowne, and H. E. Smith, 1977, Astrophys. J. Suppl. Ser. 33, 113.

Burbidge, G. R., T. W. Jones, and S. L. O'Dell, 1974, Astrophys. J. 193, 43.

Burbidge, G. R., and J. Perry, 1976, Astrophys. J. Lett. 205, L55.

Burgess, A., 1964, Mem. R. Astron. Soc. 69, 1.

Burgess, A., 1965, Astrophys. J. 141, 1588.

Burgess, A., and M. J. Seaton, 1960, Mon. Not. R. Astron. Soc. 121, 471 .

Burgess, A., H. P. Summers, D. M. Cochrane, and R. W. P. McWhirter, 1977, Mon. Not. R. Astron. Soc. 179, 275. Burgess, D. D., 1967, Nature (Lond.) 216, 1092.

Burke, P. G., 1972, in Atoms and Molecules in Astrophysics, edited by T. R. Carson and M. J. Roberts (Academic, London), p. 1.

Burke, P. G., J. W. Cooper, and S. Ormonde, 1969, Phys. Rev. 183, 245.

Burke, P. G., and D. L. Moores, 1968, J. Phys. B 1, 575. Burstein, D., and C. Heiles, 1978a, Astrophys. Lett. 19, 69. Burstein, D., and C. Heiles, 1978b, Astrophys. J. 225, 40. Butler, S. E., S. L. Guberman, and A. Dalgarno, 1977, Phys. Rev. A 16, 500 .

Canto, J., and E. Daltabuit, 1974, Rev. Mex. Astron. Astrof. 1,5 .

Capriotti, E. R., 1964a, Astrophys. J. 139, 225.

Capriotti, E. R., 1964b, Astrophys. J. 140, 632.

Capriotti, E. R., 1965, Astrophys. J. 142, 1101.

Capriotti, E. R., 1966, Astrophys. J. 146, 709.

Capriotti, E. R., C. Foltz, and P. Byard, 1979, Astrophys. J. 230, 681 .

Chamberlain, J. W., 1956, Astrophys. J. 124, 390.

Chan, Y.-W. T., and E. M. Burbidge, 1975, Astrophys. J. 198, 45.

Chapman, R. D., and R. J. W. Henry, 1971, Astrophys. J. 168, 169.

Chapman, R. D., and R. J. W. Henry, 1972, Astrophys. J. 173, 243.

Christensen, A. B., 1979, Astrophys. J. 229, 448.

Christensen, R. B., W. D. Watson, and R. J. Blint, 1977, Astrophys. J. 213, 712 .

Coleman, G., R. F. Carswell, P. A. Strittmatter, R. E. Williams, J. A. Baldwin, L. B. Robinson, and E. J. Wampler, 1976, Astrophys. J. 207, 1.

Collin-Souffrin, S., 1978, Phys. Scr. 17, 293.

Collin-Souffrin, S., M. Joly, N. Heidmann, and S. Dumont, 1979, Astron. Astrophys. 72, 293.

Combet Farnoux, F., and M. Lamoureux, 1976, J. Phys. B $9,897$.

Conneely, M. J., K. Smith, and L. Lipsky, 1970, J. Phys. B
$3,493$.

Cox, D. P., and E. Daltabuit, 1971, Astrophys. J. 167, 113.

Cox, D. P., and W. G. Mathews, 1969, Astrophys. J. 155, 859. Crandall, D. H., G. H. Dunn, A. Gallagher, D. G. Hummer, C. V. Kunasz, D. Leep, and P. O. Taylor, 1974, Astrophys. J. 191, 789.

Culhane, J. L., 1978, Q. J. R. Astron. Soc. 19, 1.

Czyzak, S. J., and T.K. Krueger, 1979, Astrophys. Space. Sci. $60,1$.

Czyzak, S. J., T. K. Krueger, P. de A. P. Martins, H. E. Saraph, and M. J. Seaton, 1970, Mon. Not. R. Astron. Soc. 148, 361 .

Czyzak, S. J., T. K. Krueger, P. de A. P. Martins, H. E. Saraph, M. J. Seaton, and J. Shemming, 1968, in Planetary Nebulae, IAU Symp. 34, edited by D. E. Osterbrock and C. R. O'Dell (Reidel, Dordrecht), p. 138.

Dalgarno, A., 1978, in Planetary Nebulae, IAU Symp. 76, edited by Y. Terzian (Reidel, Dordrecht), p. 139.

Dalgarno, A., and S. E. Butler, 1978, Comments At. Mol. Phys. 7, 129.

Dalgarno, A., and R. A. McCray, 1972, Annu. Rev. Astron. Astrophys. 10, 375.

Daltabuit, E., and D. P. Cox, 1972a, Astrophys. J. Lett. 173, L13.

Daltabuit, E., and D. P. Cox, 1972b, Astrophys. J. 177, 855.

Daltabuit, E., G. M. MacAlpine, and D. P. Cox, 1978, Astrophys. J. 219, 372.

Daum, G. R., and H. P. Kelly, 1976, Phys. Rev. A 13, 715.

Davidsen, A. F., W. G. Fastie, and G. F. Hartig, 1977, Nature (Lond.) 269, 203.

Davidson, K., 1972, Astrophys. J. 171, 213.

Davidson, K., 1973, Astrophys. J. 181, 1; 186, 399(E) (1973).

Davidson, K., 1975, Astrophys. J. 195, 285.

Davidson, K., 1976, Astrophys. J. 207, 710.

Davidson, K., 1977a, Astrophys. J. Lett. 213, L31.

Davidson, K., 1977b, Astrophys. J. 218, 20.

Davidson, K., 1978, Bull. Am. Astron. Soc. 9, 630.

Davis, J., 1974, J. Quant. Spectrosc. Radiat. Transfer 14, 549.

Dibai, E. A., and V. F. Esipov, 1967, Sov. Astron.-AJ 11, 43. DiCocco, G., G. Boella, F. Perotti, R. Stiglitz, G. Villa, R. E. Baker, R. C. Butler, A. J. Dean, S. J. Martin, and D. Ramsden, 1977, Nature (Lond.) 270, 319.

Disney, M. J., 1973, Astrophys. J. Lett. 181, L55.

Drake, G. W. F., 1971, Phys. Rev. A 3, 908.

Drake, G. W. F., G. A. Victor, and A. Dalgarno, 1969, Phys. Rev. 180, 25.

Drew, J. E., 1978, Mon. Not. R. Astron. Soc. 185, 193.

Eachus, L. J., and W. Liller, 1975, Astrophys. J. Lett. 200, L61.

Eilik, J. A., and L. J. Caroff, 1976, Astrophys. J. 208, 887. Elias, J. H., D. J. Ennis, D. Y. Gezari, M. G. Hauser, J. R. Houck, K. Y. Lo, K. Matthews, D. Nadeau, G. Neugebauer, M. W. Werner, and W. E. Westbrook, 1978, Astrophys. J. $220,25$.

Elvis, M., T. Maccacaro, A. S. Wilson, M. J. Ward, M. V. Penston, R. A. E. Fosbury, and G. C. Perola, 1978, Mon. Not. R. Astron. Soc. 183, 12.

Felden, M. M., and M. A. Felden, 1972, Astrophys. J. 174, 219.

Feldman, F. R., 1978, Ph.D. thesis, Univ, of Michigan.

Feldman, F. R., and G. M. MacAlpine, 1978, Astrophys. J. 221, 486.

Feltz, K. A., 1972, Publ. Astron. Soc. Pac. 84, 497.

Ferland, G. J., and H. Netzer, 1979, Astrophys. J., 229, 274.

Ferland, G. J., H. Netzer, and G. A. Shields, 1979, Astrophys. J. in press.

Field, G. B., H. Arp, and J. N. Bahcall, 1973, editors The Redshift Controversy, (Benjamin, Reading, Mass.). Field, G. B., and G. Steigman, 1971, Astrophys. J. 166, 59. Flannery, M. R., and K. J. McCann, 1975, Phys. Rev. A 12, 846. 
Flower, D. R., 1969, Mon. Not. R. Astron. Soc. 146, 171. Flower, D. R., and J. M. Launay, 1972, J. Phys. B 5, L207. Flower, D. R., and J. M. Launay, 1973, Astron. Astrophys. 29, 321 .

Flower, D. R., and H. Nussbaumer, 1975a, Astron. Astrophys. $42,265$.

Flower, D. R., and H. Nussbaumer, 1975b, Astron. Astrophys. 45, 145.

Franco, J., and E. Daltabuit, 1978, Rev. Mex. Astron. Astrof. $2,325$.

Garstang, R. H., 1968, in Planetary Nebulae, IAU Symp. 34, edited by D. E. Osterbrock and C. R. O'Dell (Reidel, Dordrecht), p. 143.

Garstang, R. H., W. D. Robb, and S. P. Rountree, 1978, Astrophys. J. 222, 384.

Geltman, S., and P. G. Burke, 1970, J. Phys. B 3, 1062.

Geltman, S., M. R. H. Rudge, and M. J. Seaton, 1963, Proc.

Phys. Soc. Lond. 81, 375.

Giacconi, R., 1978, Phys. Scr. 17, 159.

Giles, K., 1977, Mon. Not. R. Astron. Soc. 180, $57 \mathrm{P}$.

Ginzburg, V. L., and L. M. Ozernoi, 1977, Astrophys. Space Sci. 48, 401.

Glasco, H. P., and H. Zirin, 1964, Astrophys. J. Suppl. 9, 193. Goodson, W. L., 1967, Z. Astrophys. 66, 118.

Gottlieb, E. W., and W. Liller, 1978, Astrophys. J. Lett. 222, L1.

Gould, R. J., 1978, Astrophys. J. 219, 250.

Grandi, S. A., 1975, Astrophys. J. 196, 465.

Grandi, S. A., 1978. Astrophys. J. 221, 501.

Grandi, S. A., and D. E. Osterbrock, 1978, Astrophys.

J. 220, 783.

Grandi, S. A., and M. M. Phillips, 1978, Astrophys. J. 220, 426.

Grandi, S. A., and W. G. Tifft, 1974, Publ. Astron. Soc. Pac. $86,873$.

Grasdalen, G. L., 1976, Astrophys. J. Lett. 208, L11.

Green, R. F., T. B. Williams, and D. C. Morton, 1978, Astrophys. J. 226, 729.

Greenstein, J. L., and M. Mathews, 1963, Nature (Lond.) 197, 1041.

Greenstein, J. L., and M. Schmidt, 1964, Astrophys. J. 140, 1.

Griem, H. R., 1969, Astrophys. J. Lett. 156, L103.

Gursky, H., and D. A. Schwartz, 1977, Annu. Rev. Astron.

Astrophys. 15, 541.

Harrington, J. P., 1968, Astrophys. J. 152, 943.

Hawkins, M. R. S., 1978, Mon. Not. R. Astron. Soc. 182, 361.

Heckman, T. M., 1976, Publ. Astron. Soc. Pac. 88, 844.

Henry, R. J. W., 1970, Astrophys. J. 161, 1153.

Henyey, L. G., 1940, Proc. Natl. Acad. Scị. USA 96, 794.

Heroux, L., 1967, Phys. Rev. 153, 156.

Heroux, L., 1969, Phys. Rev. 180, 1.

Hewitt, T. G., and P. D. Noerdlinger, 1974, Astrophys. J. 188, 315.

Hidalgo, M. B., 1968, Astrophys. J. 153, 981.

Hildebrand, R. H., S. E. Whitcomb, R. Winston, R. F. Stien-

ing, D. A. Harper, and S. H. Moseley, 1977, Astrophys. J. $216,698$.

Hills, J. G., 1978, Mon. Not. R. Astron. Soc. 182, 517.

Hjellming, R. M., 1966, Astrophys. J. 143, 420.

Holmberg, E. B., 1974, Astron. Astrophys. 35, 121.

Holstein, T., 1947, Phys. Rev. 72, 1212.

Huffman, D. R., 1976, in Solid State Astrophysics, edited by

N. C. Wickramasinghe and D. J. Morgan (Reidel, Dordrecht), p. 191.

Huffman, D. R., 1977, Adv. Phys. 26, 129.

Hummer, D. G., and P. B. Kunasz, 1979, preprint.

Hummer, D. G., and M. J. Seaton, 1963a, Mon. Not. R. Astron. Soc. 125, 437.

Hummer, D. G., and M. J. Seaton, 1963b, Mon. Not. R. Astron. Soc. 125, 461.

Hummer, D. G., and M. J. Seaton, 1964, Mon.'Not. R. As- tron. Soc. 127, 217.

Hyland, A. R., E. E. Becklin, and G. Neugebauer, 1978, Astrophys. J. Lett. 220, L73.

Jackson, A. R. G., 1973, Mon. Not. R. Astron. Soc. 165, 53.

Jones, T. W., C. W. Leung, R. J. Gould, and W. A. Stein,

1977, Astrophys. J. 212, 52.

Jones, T. W., S. L. O'Dell, and W. A. Stein, 1974, Astrophys. J. 192, 261 .

Jura, M., 1973, Astrophys. J. 181, 627.

Karzas, W. J., and R. Latter, 1961, Astrophys. J. Suppl. Ser. 6,167 .

Katz, A., 1975, Astrophys. J. 198, 255.

Keenan, D. W., 1978, Mon. Not. R. Astron. Soc. 185, 389.

Kellerman, K. I., 1974, in Galactic and Extragalactic Radio

Astronomy, edited by G. L. Verschuur and K. I. Kellerman

(Springer, New York), p. 320.

Kellerman, K. I., 1978, Phys. Scr. 17, 257.

Khare, S. P., and B. D. Padalia, 1970, J. Phys. B 3, 1073.

Kimmer, E., 1976, Astrophys. J. 203, 674.

Kinman, T. D., E. Lamla, and C. A. Wirtanen, 1966, Astrophys. J. 146, 964.

Kippenhahn, R., 1977, Astron. Astrophys. 55, 125.

Kippenhahn, R., 1978, Phys. Scr. 17, 301.

Kippenhahn, R., L. Mestel, and J. J. Perry, 1975, Astron.

Astrophys. 44, 123.

Kippenhahn, R., J. J. Perry, and H. J. Röser, 1974, Astron.

Astrophys. 34, 211.

Knude, J. K., 1977, Astrophys. Lett. 18, 115.

Koelblad, D., 1956, Bull. Astron. Inst. Neth. 12, 341.

Kristian, J., 1973, Astrophys. J. Lett. 179, L61.

Krolik, J. H., 1979, Astrophys. J. 228, 13.

Krolik, J. H., and C. F. McKee, 1978, Astrophys. J. Suppl. Ser. 37, 459.

Krolik, J. H., C. F. McKee, and C. B. Tarter, 1978, in Proceedings of the Pittsburgh Conference on BL Lacertae Objects, edited by A. Wolfe (University of Pittsburgh, Pitts burgh), p. 277.

Kunc, J., and M. Zgorzelski, 1975, At. Data Nucl. Data Tables 15, 543.

Levich, E. V., and R. A. Sunyaev, 1970, Astrophys. Lett. 7, 69.

Liller, M. H., and W. Liller, 1975, Astrophys. J. Lett. 199, L133.

London, R., 1979, Astrophys. J. 228, 8.

Loulergue, M., and H. Nussbaumer, 1976, Astron. Astrophys. $51,163$.

Lovelace, R. V. E., 1976, Nature (Lond.) 262, 649.

Lynden-Bell, D., 1969, Nature (Lond.) 223, 690.

Lynden-Bell, D., 1978, Phys. Scr. 17, 185.

Lynden-Bell, D., and M. J. Rees, 1971, Mon. Not. R. Astron. Soc. $152,461$.

Lynds, C. R., 1967, Astrophys. J. 147, 396.

Lynds, C. R., and D. Wills, 1972, Astrophys. J. 172, 531.

Lyutyi, V. M., 1977, Sov. Astron-AJ 21, 655.

MacAlpine, G. M., 1972, Astrophys. J. 175, 11.

MacAlpine, G. M., 1974, Astrophys. J. 193, 37.

MacAlpine, G. M., 1976, Astrophys. J. 204, 694.

Marti, F., and P. D. Noerdlinger, 1977, Astrophys. J. 215, 247.

Malinovsky, M., 1975, Astron. Astrophys. 43, 101.

Marr, G. V., and J. B. West, 1976, At. Data Nucl. Data Tables $18,498$.

Marscher, A. P., 1978, Astrophys. J. 225, 725.

Marshall, F. E., R. F. Mushotzky, E. A. Boldt, S. S. Holt, R. E. Rothchild, and P. J. Serlemitsos, 1978, Nature (Lond.) 275,624 .

Mathews, w. G., 1974, Astrophys. J. 189, 23.

Mathews, W. G., and G. R. Blumenthal, 1977, Astrophys. J. $214,10$.

Mathis, J. S., 1970, Astrophys. J. 162, 761.

McGimsey, B. Q., A. G. Smith, R. L. Scott, R. J. Leacock,

P. L. Edwards, R. L. Hackney, and K. R. Hackney, 1975, 
Astron. J. 80, 895.

McKee, C. F., and V. Petrosian, 1974, Astrophys. J. 189, 17. McKee, C. F., and C. B. Tarter, 1975, Astrophys. J. 202, 306.

McWhirter, R. W. P., 1975, in Atomic and Molecular Processes in Astrophysics, edited by M. C. E. Huber and H. Nussbaumer (Geneva Observatory, Saverny, Switzerland), p. 187.

Menzel, D. H., 1962, Selected Papers on Physical Processes in Ionized Plasmas, editor (Dover, New York).

Menzel, D. H., and C. L. Pekeris, 1935, Mon. Not. R. Astron. Soc. 96, 77 .

Mergenthaler, J. L., 1975, Astrophys. J. 199, 770.

Mestel, L., D. W. Moore, and J. J. Perry, 1976, Astron. Astrophys. 52, 203.

Mewe, R., 1972, Astron. Astrophys. 20, 215.

Mezger, P. G., L. F. Smith, and E. Churchwell, 1974, Astron. Astrophys. 32, 269.

Miley, G. K., and J. S. Miller, 1979, Astrophys. J. Lett. 228, L55.

Miller, J. S., 1978, Comments Astrophys. 7, 175.

Moiseiwitsch, B. L., and S. J. Smith, 1968, Rev. Mod. Phys. $40,238$.

Moores, D. L., and H. Nussbaumer, 1970, J. Phys. B 3, 161.

Morton, D. C., and W. H. Smith, 1973, Astrophys. J. Suppl. Ser. 26, 333 .

Morton, D. C., T. B. Williams, and R. F. Green, 1978, Astrophys. J. 219, 381.

Murdoch, H. S., and D. F. Crawford, 1977, Mon. Not. R. Astron. Soc. 180, 41P

Mushotsky, R. F., P. M. Solomon, and P. A. Strittmatter, 1972, Astrophys. J. 174, 7.

Netzer, H., 1974, Mon. Not. R. Astron. Soc. 169, 579.

Netzer, H., 1975, Mon. Not. R. Astron. Soc. 171, 395.

Netzer, H., 1976, Mon. Not. R. Astron. Soc. 177, 473.

Netzer, H., 1977a, Mon. Not. R. Astron. Soc. 178, 489.

Netzer, H., 1977b, Mon. Not. R. Astron. Soc. 181, 89P.

Netzer, H., 1978, Astrophys. J. 219, 822.

Netzer, H., and K. Davidson, 1979, Mon. Not. R. Astron. Soc., 187, 871.

Netzer, H., and M. V. Penston, 1976, Mon. Not. R. Astron.

Soc. 174, 319 .

Neugebauer, G., 1978, Phys. Scr. 17, 149

Neugebauer, G., J. B. Oke, E. E. Becklin, and K. Matthews, 1979, Astrophys. J., 230, 79.

Ney, E. P., 1977, Science 195, 541.

Noerdlinger, P. D., and G. B. Rybicki, 1974, Astrophys. J.

$193,651$.

Nussbaumer, H., 1971, Astrophys. J. 166, 411.

Nussbaumer, H., 1972, Astron. Astrophys. 16, 77.

Nussbaumer, H., and D. E. Osterbrock, 1970, Astrophys. J. $161,811$.

Nussbaumer, H., and C. Rusca, 1979, Astron. Astrophys. 72, 129.

Nussbaumer, H., and P. J. Storey, 1978, Astron. Astrophys., 64, 139.

Oke, J. B., 1963, Nature (Lond.) 197, 1040

Oke, J. B., 1967, Astrophys. J. 147, 901.

Oke, J. B., 1974, Astrophys. J. Lett. 189, L47.

Oke, J. B., G. Neugebauer, and E. E. Becklin, 1970, Astrophys. J. 159, 341 .

Oke, J. B., and W. L. W. Sargent, 1968, Astrophys. J. 151, 807.

Oke, J. B., and G. A. Shield, 1976, Astrophys. J. 207, 713.

Opher, R., 1974, Astrophys. J. 187, 5.

Osmer, P. S., 1977, Astrophys. J. 214, 1.

Osmer, P. S., 1979, Astrophys. J. 227, 18.

Osmer, P. S., M. G. Smith, and D. W. Weedman, 1974, Astrophys. J. 189, 187.

Osmer, P. S., and M. G. Smith, 1976, Astrophys. J. 210, 267.

Osmer, P. S., and M. G. Smith, 1977a, Astrophys. J. 213, 607.

Osmer, P. S., and M. G. Smith, 1977b, Astrophys. J. Lett.
215, L47.

Osterbrock, D. E., 1962, Astrophys. J. 135, 195.

Osterbrock, D. E., 1970a, Astrophys. J. 160, 25.

Osterbrock, D. E., 1970b, J. Phys. B 3, 149.

Osterbrock, D. E., 1974, Astrophysics of Gaseous Nebulae

(Freeman, San Francisco).

Osterbrock, D. E., 1976, Astrophys. J. 203, 329.

Osterbrock, D. E., 1977, Astrophys. J. 215, 733.

Osterbrock, D. E., 1978a, Phys. Scr. 17, 137.

Osterbrock, D. E., 1978b, Phys. Scr. 17, 285.

Osterbrock, D. E., 1978c, preprint.

Osterbrock, D. E., and S. A. Grandi, 1979, Astrophys. J.

Lett. 228, L59.

Osterbrock, D. E., and A. T. Koski, 1976, Mon. Not. R. As-

tron. Soc. 176, 61P.

Osterbrock, D. E., and R. A. R. Parker, 1965, Astrophys. J. 141, 892 .

Osterbrock, D. E., and R. A. R. Parker, 1966, Astrophys. J. 143, 268.

Osterbrock, D. E., and M. M. Phillips, 1977, Publ. Astron.

Soc. Pac. 89, 251

Osterbrock, D. E., and R. K. Wallace, 1977, Astrophys. Lett. $19,11$.

Pagel, B. E. J., 1976, in The Galaxy and the Local Group, edited by R. J. Dickens and J. E. Perry (R. Greenwich Obs. Bull. 182), p. 65.

Pagel, B. E. J., M. G. Edmunds, R. A. E. Fosbury, and B. L. Webster, 1978, Mon. Not. R. Astron. Soc. 184, 569.

Panagia, N., and M. Ranieri, 1973, Astron. Astrophys. 24, 219.

Panagia, N., and L. F. Smith, 1978, Astron. Astrophys. 62, 277.

Parker, R. A. R., 1964, Astrophys. J. 139, 208.

Pastoriza, M., and H. Gerola, 1970, Astrophys. Lett. 6, 155.

Peach, G., 1962, Mon. Not. R. Astron. Soc. 124, 371.

Peach, G., 1967, Mem. R. Astron. Soc. 71, 13.

Peimbert, M., 1975, Annu. Rev. Astron. Astrophys. 13, 113.

Perry, J.J., E. M. Burbidge, and G. R. Burbidge, 1978, Publ. Astron. Soc. Pac. 90, 337.

Pfleiderer, J., and M. Grewing, 1967, Science 157, 544.

Phillips, M. M., 1978a, Astrophys. J. Suppl. Ser. 38, 187.

Phillips, M. M., 1978b, Astrophys. J. 226, 736.

Phillips, M. M., 1979, Astrophys. J. Suppl. Ser. 39, 377.

Pradhan, A. K., 1974, J. Phys. B 7, L503.

Pradhan, A. K., 1976, Mon. Not. R. Astron. Soc. 177, 31.

Pradhan, A. K., 1978, Mon. Not. R. Astron. Soc. 183, 89P.

Ptak, R., and R. E. Stoner, 1973a, Astrophys. J. Lett. 179, L89.

Ptak, R., and R. E. Stoner, 1973b, Astrophys. J. 185, 121.

Ptak, R., and R. Stoner, 1975, Astrophys. J. 200, 558.

Ptak, R., and R. Stoner, 1976, Astrophys. J. 210, 25.

Ptak, R., and R. Stoner, 1977, Astrophys. J. 217, 940.

Puetter, R. C., H. E. Smith, and S. P. Willner, 1979, Astro-

phys. J. Lett. 227, L5.

Puetter, R. C., H. E. Smith, B. T. Soifer, S. P. Willner, and

J. L. Pipher, 1978, Astrophys. J. Lett. 226, L53.

Purcell, E. M., 1952, Astrophys. J. 116, 457.

Rau, A. R. P., 1971, Phys. Rev. A 4, 207.

Raymond, J. C., and B. Smith, 1977, Astrophys. J. Suppl. Ser. $35,419$.

Rees, M. J., 1977a, Q.J. R. Astron. Soc. 18, 429.

Rees, M. J., 1977b, Ann. N. Y. Acad. Sci. 302, 613.

Rees, M. J., 1978a, Phys. Scr. 17, 193.

Rees, M. J., 1978b, Observatory 98, 210.

Rees, M. J., J. I. Silk, M. W. Werner, and N. C. Wickramasinghe, 1969, Nature (Lond.), 223, 788.

Richstone, D. O., and J. B. Oke, 1977, Astrophys. J. 213, 8.

Rieke, G. H., 1978, Astrophys. J. 226, 550.

Robbins, R. R., 1968, Astrophys. J. 151, 497.

Robbins, R. R., 1970, Astrophys. J. 160, 519.

Robbins, R. R., and E. L. Robinson, 1971, Astrophys. J. 167, 249. 
Roberts, D. E., 1970, J. Phys. B 3, 676.

Rowan-Robinson, M., 1976, Nature (Lond.) 262, 97.

Rowan-Robinson, M., 1977, Astrophys. J. 213, 635.

Rowe, D., R. Stoner, and R. Ptak, 1978, Astrophys. J. 224,

761 .

Rubin, R. H., 1968, Astrophys. J. 153, 761.

Rudge, M. R. H., 1968, Rev. Mod. Phys. 40, 564

Sakhibullin, N., and A. J. Willis, 1978, Astron. Astrophys.

Suppl. 31, 11.

Salpeter, E. E., 1977, Annu. Rev. Astron. Astrophys. 15, 267.

Sandage, A., 1972, Astrophys. J. 178, 25.

Sandage, A., 1973, Astrophys. J. 183, 711.

Sandage, A., and W. J. Luyten, 1969, Astrophys. J. 155, 913.

Sandage, A. R., J. A. Westphal, and P. A. Strittmatter, 1966, Astrophys. J. 146, 322.

Saraph, H. E., and M. J. Seaton, 1970, Mon. Not. R. Astron. Soc. 148, 367.

Sargent, W. L. W., 1968, Astrophys. J. Lett. 152, L31.

Sargent, W. L. W., T. A. Boroson, 1979, Astrophys. J. 228, 712

Scargle, J., L. Caroff, and P. Noerdlinger, 1970, Astrophys. J. Lett. 161, L115.

Scargle, J. D., L. J. Caroff, and C. B. Tarter, 1974, AstroPhys. J. 189, 181.

Schild, R. E., 1977, Astron. J. 82, 337.

Schmidt, K.-H., 1976, in Solid State Astrophysics, edited by N. C.

Wickramasinghe and D. J. Morgan (Reidel, Dordrecht), p. 23.

Schmidt, M., 1963, Nature (Lond.) 197, 1040.

Schmidt, M., 1964, in Quasars and High Energy Astronomy,

2nd Texas Symposium on Relativistic Astrophysics, edited

by K. N. Douglas, et al. (Gordon and Breach, New York).

Schmidt, M., 1969, Annu. Rev. Astron. Astrophys. 7, 527.

Schmidt, M., 1970, Astrophys. J. 162, 371.

Schmidt, M., 1972, Astrophys. J. 176, 273.

Scott, R. L., R. J. Leacock, B. Q. McGimsey, A. G. Smith,

P. L. Edwards, K. R. Hackney, and R. L. Hackney, 1976, Astron. J. 81, 7.

Searle, L., 1976, in The Galaxy and the Local Group, edited by R. J. Dickens and J. E. Perry (R. Greenwich Obs. Bull. 182), p. 119

Searle, L., and W. L. W. Sargent, 1968, Astrophys. J. 153, 1003.

Seaton, M. J., 1955, Proc. Phys. Soc. Lond. A 68, 457.

Seaton, M. J., 1958, Rev. Mod. Phys. 30, 979.

Seaton, M. J., 1959, Mon. Not. R. Astron. Soc. 119, 81.

Seaton, M. J., 1960, Rep. Prog. Phys. 23, 313.

Seaton, M. J., 1972, in Atoms and Molecules in Astrophysics, edited by T. R. Carson and M. J. Roberts (Academic, London), p. 121.

Seaton, M. J., 1975, Mon. Not. R. Astron. Soc. 170, 475.

Seaton, M. J., 1978, Mon. Not. R. Astron. Soc. 185, 5P

Shapiro, J., and G. Breit, 1959, Phys. Rev. 113, 179.

Shevelko, V. P., 1974, Opt. Spectrosc. 36, 7 .

Shields, G. A., 1974, Astrophys. J. 191, 309.

Shields, G. A., 1976, Astrophys. J. 204, 330.

Shields, G. A., 1977, Astrophys. Lett. 18, 119

Shields, G. A., 1978, in Proceedings of the Pittsburgh Conference on BL Lacertae Objects, edited by A. Wolfe (University of Pittsburgh, Pittsburgh), p. 257.

Shields, G. A., and J. B. Oke, 1975, Astrophys. J. 197, 5.

Shields, G. A., J. B. Oke, and W. L. W. Sargent, 1972. Astrophys. J. 176, 75 .

Shields, G. A., and L. Searle, 1978, Astrophys. J. 222, 821.

Shklovsky, I. S., 1965, Sov. Astron.-AJ 8, 638.

Shuder, J. M., and G. M. MacAlpine, 1979, Astrophys. J. 230 , 348 .

Shull, J. M., 1979, Astrophys. J., in press.

Shull, J. M., and R. McCray, 1978, Astrophys. J. Lett. 223, L5.

Shull, J. M., and C. F. McKee, 1979, Astrophys. J., in press.

Silk, J., H. E. Spinrad, and G. B. Field, 1973, Astrophys. J.

Lett. 181, L25.
Smith, M. G., 1978, Vistas in Astronomy 22, 321.

Soifer, B. T., J. B. Oke, K. Y. Mathews, and G. Neugebauer, 1979, Astrophys. J. Lett. 227, L1.

Souffrin, S., 1969a, Astron. Astrophys. 1, 305.

Souffrin, S., 1969b, Astron. Astrophys. 1, 414.

Souffrin, S. C., D. Alloin, and Y. Andrillat, 1973, Astron.

Astrophys. 22, 343.

Spitzer, L., 1962, Physics of Fully Ionized Gases (Interscience, New York).

Spitzer, L., 1968, Diffuse Matter in Space (Wiley-Interscience, New York).

Spitzer, L., 1978, Physical Processes in the Interstellar

Medium (Wiley-Interscience, New York).

Spitzer, L., and J. L. Greenstein, 1951, Astrophys. J. 114, 407.

Spitzer, L., and E. H. Scott, 1969, Astrophys. J. 158, 161.

Spitzer, L., and M. G. Tomasko, 1968, Astrophys. J. 152, 971.

Sramek, R. A., and D. W. Weedman, 1978, Astrophys. J. 221, 468.

Stark, J. P., J. B. Burnell, and J. L. Culhane, 1978, Mon. Not. R. Astron. Soc. 182, $23 \mathrm{P}$.

Steigman, G., 1975, Astrophys. J. 199, 642

Stein, W. A., S. L. O'Dell, and P. A. Strittmatter, 1976, Annu. Rev. Astron. Astrophys, 14, 173.

Stockman, H. S., and J. R. P. Angel, 1978, Astrophys. J. Lett. 220, L67.

Stockman, H. S., J. R. P. Angel, and E. A. Beaver, 1976, Bull. Am. Astron. Soc. 8, 495.

Stockman, H. S., J. R. P. Angel, and G. K. Miley, 1979, Astrophys. J. Lett. 227, L55.

Stockton, A., 1976, Astrophys. J. Lett. 205, L113.

Stoner, R. E., and R. Ptak, 1977, Astrophys. J. 214, 653.

Stoner, R. E., R. Ptak, and D. Ellis, 1974, Astrophys. J. 191, 291.

Strittmatter, P. A., and R. E. Williams, 1976, Annu. Rev. Astron. Astrophys. 14, 307.

Swanenenberg, B. N., K. Bennett, G. F. Bignami, P. Caraveo, W. Hermsen, G. Kaubach, J. L. Masnou, H. A. Mayer-Hasselwander, J. A. Paul, B. Sacco, L. Scarsi, and R. D. Wills. 1978, Nature (Lond.) 275, 298.

Tarter, C. B., 1971, Astrophys. J. 168, 313; 181, 607 (E) (1973).

Tarter, C. B., and C. F. McKee, 1973, Astrophys. J. Lett. 186, L63.

Tarter, C. B., and E. E. Salpeter, 1969, Astrophys. J. 156 , 953.

Tarter, C. B, W. H. Tucker, and E. E. Salpeter, 1969, Astrophys. J. 156, 943.

Tarter, C. B., J. C. Weisheit, and A. Dalgarno, 1979, Astron. Astrophys. 71, 366.

Thackeray, A. D., 1967, Mon. Not. R. Astron. Soc. 135, 51. Tohline, J. E., and D. E. Osterbrock, 1976, Astrophys. J. Lett. 210, L117.

Tucker, W. H., and C. B. Tarter, 1968, Astron. J. 73, 901. Turner, E. L., 1979, Astrophys. J. Lett. 228, L51.

Ulrich, M. -H., 1972a, Astrophys. J. Lett. 171, L37.

Ulrich, M. -H., 1972b, Astrophys. J. 174, 483.

Ulrich, M. -H., 1976, Astrophys. J. Lett. 207, L73.

Underhill, A. B., 1966, The Early Type Stars (Reidel, Dordrecht).

Unno, W., 1952, Publ. Astron. Soc. Jpn. 4, 100.

Uomoto, A. K., B. J. Wills, and D. Wills, 1976, Astron. J. $81,905$.

Victor, G. A., and E. R. Constantinides, 1979, preprint.

Viotti, R., 1976, Astrophys. J. 204, 293.

Von Hoerner, S., and W. C. Saslaw, 1976, Astrophys. J. 206, 917.

Wampler, E. J., 1967, Astrophys. J. 147, 1.

Wampler, E. J., 1971, Astrophys. J. 164, 1.

Wampler, E. J., and J. B. Oke, 1967, Astrophys. J. 148, 695

Wampler, E. J., L. B. Robinson, E. M. Burbidge, and J. A. 
Baldwin, 1975, Astrophys. J. Lett. 198, L49.

Weedman, D. W., 1976a, Q. J. R. Astron. Soc. 17, 227.

Weedman, D. W., 1976b, Astrophys. J. 208, 30.

Weedman, D. W., 1977, Annu. Rev. Astron. Astrophys. 15, 69

Weedman, D. W., D. W. Lewis, and G. M. MacAlpine, 1979, Astrophys. J., in press.

Wehinger, P. A., and S. Wyckoff, 1978, Mon. Not. R. Astron. Soc. 184, 335 .

Weisheit, J. C., 1974, Astrophys. J. 190, 735.

Weisheit, J. C., and L. A. Collins, 1976, Astrophys. J. 210, 299.

Weymann, R., 1970, Astrophys. J. 160, 31.

Weymann, R. J., 1973, Comments Astrophys. Space Sci. 5, 139.

Weymann, R. J., and R. E. Williams, 1969, Astrophys. J. 157 1201.

Wickramasinghe, N. C., and K. Nandy, 1972, Rep. Prog. Phys. 35, 157.

Wiese, W. L., M. W. Smith, and B. M. Glennon, 1966, Atomic Transition Probabilities: Vol. I, Hydrogen through Neon, Natl. Stand. Ref. Data Ser. Natl. Bur. Stand. 4 (U.S. GPO,
Washington D. C.).

Williams, R. E., 1967, Astrophys. J. 147, 556.

Williams, R. E., 1971, Astrophys. J. Lett. 167, L27.

Williams, R. E., 1972, Astrophys. J. 178, 105.

Williams, R. E., and R. J. Weymann, 1968, Astron. J. 73, 895.

Wills, D., 1976, Observatory 96, 145.

Wright, A. E., B. A. Peterson, D. L. Jauncey, and J. J. Condon, 1978, Astrophys. J. Lett. 226, L61.

Wright, E. L., and D. E. Kleinmann, 1978, Nature (Lond.) 275, 298.

Wu, C. C., 1977, Astrophys. J. Lett. 217, L117.

Yankulov, I. M., 1974, Sov. Astron.-AJ 18, 720.

Young, P. J., G. A. Shields, and J. C. Wheeler, 1977, Astrophys. J. 212, 367.

Zanstra, H., 1949, Bull. Astron. Inst. Neth. 11, 1.

Zanstra, H., 1951, Bull. Astron. Inst. Neth. 11, 359.

Zeippen, C. J., M. J. Seaton, and D. C.Morton, 1977, Mon. Not. R. Astron. Soc. 181, 527.

Zirin, H., 1978, Astrophys. J. Lett. 222, L105; 227, L111 (E) (1979). 